\title{
Aeronomy of the Venus Upper Atmosphere
}

\author{
J.-C. Gérard ${ }^{1}$ · S.W. Bougher ${ }^{2}$ - M.A. López-Valverde ${ }^{3}$. \\ M. Pätzold ${ }^{4}$ • P. Drossart ${ }^{5}$. G. Piccioni ${ }^{6}$
}

Received: 1 December 2016 / Accepted: 15 September 2017

(C) Springer Science+Business Media B.V. 2017

\begin{abstract}
We present aeronomical observations collected using remote sensing instruments on board Venus Express, complemented with ground-based observations and numerical modeling. They are mostly based on VIRTIS and SPICAV measurements of airglow obtained in the nadir mode and at the limb above $90 \mathrm{~km}$. They complement our understanding of the behavior of Venus' upper atmosphere that was largely based on Pioneer Venus observations mostly performed over thirty years earlier. Following a summary of recent spectral data from the EUV to the infrared, we examine how these observations have improved our knowledge of the composition, thermal structure, dynamics and transport of the Venus upper atmosphere. We then synthesize progress in three-dimensional modeling of the upper atmosphere which is largely based on global mapping and observations of time variations of the nitric oxide and $\mathrm{O}_{2}$ nightglow emissions. Processes controlling the escape flux of atoms to space are described. Results based on the VeRA radio propagation experiment are summarized and compared to ionospheric measurements collected during earlier space missions. Finally, we point out some unsolved and open questions generated by these recent datasets and model comparisons.
\end{abstract}

Venus III

Edited by Bruno Bézard, Christopher T. Russell, Takehiko Satoh, Suzanne E. Smrekar and Colin F. Wilson

\section{J.-C. Gérard}

jc.gerard@ulg.ac.be

1 Laboratoire de Physique Atmosphérique et Planétaire, STAR institute, Université de Liège, Liège, Belgium

2 Department of Climate and Space Sciences and Engineering, University of Michigan, Ann Arbor, USA

3 Solar System department, Instituto de Astrofisica de Andalucía, Granada, Spain

4 Rheinische Institut für Umweltforschung an der Universität zu Köln, Abteilung Planetenforschung, Köln, Germany

5 LESIA, Observatoire de Paris, PSL Research University, CNRS, Sorbonne Université, Univ. Paris Diderot, Meudon, France

6 Instituto di Astrofisica e Planetologia Spaziali, INAF, Rome, Italy 
Keywords Venus · Aeronomy · Upper atmosphere · Airglow · Nightglow · Composition · Dynamics · Chemistry · Transport · Ultraviolet · Infrared · Visible

\section{Introduction}

The aeronomy of a planet with an atmosphere covers all phenomena related to the interface between the planet deep layers and the radiation to deep space. It is therefore a region of transition, where radiation effects dominate and transfer of energy implies multiple phenomena. Despite studies since the beginning of the space era for the Earth, and then Venus and Mars, followed by Giant Planets and other outer system objects, decades of theoretical and observational work have not solved some of the most fundamental questions, like the temperature of the exospheres or the details of the mechanisms for non-thermal escape. This led pioneers in the field like Andrew Nagy to baptize the upper atmosphere layers, usually referred as the mesospheres, an "ignorosphere" (e.g., Schunk and Nagy 1980). Through the analogies between planets, at least between terrestrial planets on one side, Giant Planets and Titan on the other side, deep studies of Venus will serve future investigators for other planets in deciphering the physical and chemical mechanisms at work. Among the phenomena under study, the connection between dynamics, radiative transfer, chemistry and photochemistry is the most important input in recent approaches, facilitated by improvements in computing facilities, and laboratory data. In particular, early 1D modeling with transport parameters simplified to eddy diffusion coefficients used in early works (e.g. Krasnopolsky 1986), are now completed by 3-D Thermospheric Global Circulation Models, with a much deeper understanding of the complexity of phenomena.

Venus is a good terrain of exercise for testing such models in a complete way. Firstly, the knowledge of its atmosphere has been obtained continuously for more than 40 years, despite a long interruption of atmospheric space mission between the Galileo flyby and Venus Express. Secondly, Venus is-beyond Earth, the densest atmosphere to test the model. It can also be seen as a prototype of a class of exoplanets, Mars and Earth being probably more marginal and rarer cases.

In terms of photochemistry, the aeronomy layers are essential parts of the chemistry of an atmosphere: on Titan for example, aerosols are built from ion chemistry in the ionosphere and photochemistry below, and the composition of the bulk atmosphere is strongly affected by these layers. Giant planets' upper atmospheres present similar phenomena, as shown for Saturn by Cassini observations. Not even mentioning the ozone layer of Earth, of vital importance for our planet, Martian chemical stability is ensured from CO recycling by aeronomic processes.

With no intrinsic magnetic field, Venus does not provide localized phenomena associated with auroral processes like the Earth; nevertheless, particle precipitations associated with photochemistry in the upper atmosphere are present, and relevant to similar problematics. Some fundamental questions related to aeronomy are, beyond the knowledge of composition, structure and dynamics of the upper layers:

- How is the energy transfer proceeding?

- What are the detailed mechanisms of escape processes?

- What influence the processes have on the deeper circulation and/or composition?

These questions will be addressed in this chapter.

The importance of the $\mathrm{O}_{2}\left({ }^{1} \Delta\right)$ emission as a tracer of the activity of Venus has been anticipated since the first detection by Connes et al. (1979) - the model proposed for the 
interpretation (night side emission from the circulation of $\mathrm{O}$ atoms in a diurnal circulation) has been remarkably confirmed by further observations. A recent field of development is the non-LTE observations of molecular emissions, in particular in $\mathrm{CO}$ and $\mathrm{CO}_{2}$. Such emissions have been observed for a long time from ground-based (as reviewed in Krasnopolsky 2014a) or space (López-Valverde et al. 2007). They were for a long time considered as a complication in planetary radiative transfer precluding inversion of spectra for composition or thermal measurements. $\mathrm{CO}_{2}$ non-LTE emission is now used as another tool for planetary scientists to sound the mesospheres of planets, although the associated diagnostics are more complex to derive than from LTE emissions.

A powerful method for sounding ionosphere and upper atmosphere since the early space exploration is the radio-occultation method: Venus Express has allowed during its long life in orbit around Venus a rebirth of this old method with results spanning the thermal profiles at various latitudes of Venus at unprecedented vertical resolution. Combining these new results of Venus Express with previous observations, and new modeling through powerful 3D chemical- dynamical models gives a new panorama of the upper atmosphere of Venus. This introduces not only a deeper knowledge of the sister planet of the Earth, but also to a better understanding of the underlying mechanisms. It paves the way of future extrapolation to exoplanets where Venus is probably a prototype of an important class of objects around other stars.

\section{Aeronomic Processes and Airglow Observations}

Aeronomic and airglow processes in the Venus upper atmosphere and developments based on laboratory measurements and observations from space missions have been previously reviewed in several articles and books. Chapter13 by von Zahn et al. (1983) in the first Venus book provided a detailed introduction to the Venus aeronomy and the advances in this field generated by the Pioneer Venus mission. A non-exhaustive list of later reviews published before and during the Venus Express era includes Fox and Dalgarno (1979), Paxton and Anderson (1992), Fox and Bougher (1991), Fox (1992), Huestis et al. (2008), Gronoff et al. (2008), Krasnopolsky (2011, 2013), Bhardwaj and Jain (2013). Remaining key questions in the field of the aeronomy of the upper atmosphere preceding the Venus Express mission were reviewed by Witasse and Nagy (2006) and Bougher et al. (2006). We first summarize the processes leading to airglow emissions, followed by a review of recent spectral observations of the Venus day and night airglow from the extreme ultraviolet to the infrared.

\subsection{Airglow Processes}

Airglow of neutral species may be produced by different photochemical processes leading to the formation of an excited atom or molecule denoted by an asterisk:

1. Resonant scattering (or fluorescence)

$$
\begin{aligned}
& \mathrm{A}+\mathrm{h} v \rightarrow \mathrm{A}^{*} \text { or } \\
& \mathrm{AB}+\mathrm{h} v \rightarrow \mathrm{AB}^{*}
\end{aligned}
$$

2. Photodissociative excitation

$$
\begin{aligned}
& \mathrm{AB}+\mathrm{h} v \rightarrow \mathrm{A}^{*}+\mathrm{B} \text { or } \\
& \mathrm{AB}+\mathrm{h} v \rightarrow \mathrm{A}+\mathrm{B}^{*}
\end{aligned}
$$


3. Photoelectron impact

$$
\begin{aligned}
& \mathrm{A}+\mathrm{e}_{\mathrm{f}} \rightarrow \mathrm{A}^{*}+\mathrm{e}_{\mathrm{f}} \quad \text { or } \\
& \mathrm{AB}+\mathrm{e}_{\mathrm{f}} \rightarrow \mathrm{A}^{*}+\mathrm{B}+\mathrm{e}_{\mathrm{f}}
\end{aligned}
$$

4. Photoelectron dissociative excitation

$$
\begin{aligned}
& \mathrm{AB}+\mathrm{e}_{\mathrm{f}} \rightarrow \mathrm{A}^{*}+\mathrm{B}+\mathrm{e}_{\mathrm{f}} \text { or } \\
& \mathrm{AB}+\mathrm{e}_{\mathrm{f}} \rightarrow \mathrm{A}+\mathrm{B}^{*}+\mathrm{e}_{\mathrm{f}}
\end{aligned}
$$

5. Dissociative recombination

$$
\mathrm{AB}^{+}+\mathrm{e}_{\mathrm{th}} \rightarrow \mathrm{A}^{*}+\mathrm{B}
$$

6. Exoenergetic chemical reaction

$$
\mathrm{B}+\mathrm{C} \rightarrow \mathrm{A}^{*}(+\mathrm{D})
$$

where $\mathrm{A}^{*}$ (or $\mathrm{AB}^{*}$ ) denotes an excited atom (molecule), $\mathrm{h} v$ a solar photon, $\mathrm{e}_{\mathrm{f}}$ an energetic (generally photo-) electron and $\mathrm{e}_{\mathrm{th}}$ a thermal electron. A detailed description of photochemical processes in $\mathrm{CO}_{2}$-dominated atmospheres was given by Fox (1992). In this section, we summarize some of the recent experimental results in different spectral ranges from the extreme ultraviolet to the infrared.

\subsection{Extreme Ultraviolet}

Ultraviolet spectra of the Venus dayside were reviewed by Paxton and Anderson (1992). Bertaux et al. (1981) reported measurements of the HI $121.6 \mathrm{~nm}, \mathrm{HeI} 58.4 \mathrm{~nm}$ and OI $130.4 \mathrm{~nm}$ emission obtained by two EUV spectrophotometers during the Venera 11 and 12 Venus flybys in December 1978. Further observations of the EUV dayside emissions between 82.5 and $111 \mathrm{~nm}$ were made by Stern et al. (1996) from a rocket-borne spectrograph at a spectral resolution of $0.64 \mathrm{~nm}$. The disk intensities of the OI, OII and HI emissions were about half of those reported from the Galileo flyby in February 1990 (Hord et al. 1991). They identified an emission near $110 \mathrm{~nm}$ as the $(0,0)$ band of the $\mathrm{CO}(\mathrm{C}-\mathrm{X})$ emission normally near $108.8 \mathrm{~nm}$. Previously unidentified NII emissions were also observed. No signature of argon emission was positively identified near the resonance lines at $86.7 \mathrm{~nm}, 104.8 \mathrm{~nm}$ or $106.6 \mathrm{~nm}$.

Spectra and global disk intensities of the dayside of the planet were obtained with the Hopkins Ultraviolet Telescope (HUT) on board the Space Shuttle ASTRO-2 mission on 13 March 1995 by Feldman et al. (2000). The spectral region from 82 to $184 \mathrm{~nm}$ was observed at a spectral resolution of $0.4-0.45 \mathrm{~nm}$. This spectral range is dominated by the CO Fourth Positive bands, the OI multiplets at 135.6 and $130.4 \mathrm{~nm}$ and the CI multiplets at 156.1 and $165.7 \mathrm{~nm}$. Several features were identified for the first time such as the CO Hopfield-Birge $\mathrm{B}-\mathrm{X}$ transition at $115.1 \mathrm{~nm}$. The brightness of the Fourth Positive CO A-X emission was in agreement with a model assuming resonance scattering and the $\mathrm{CO}$ column density given by the VIRA model. The B-X and the C-X CO bands, the NI 113.4 and $120.0 \mathrm{~nm}$ multiplets, and the CII $133.5 \mathrm{~nm}$ emissions were identified for the first time. This identification confirmed the presence of $\mathrm{C}^{+}$ions in the Venus ionosphere. As expected from the low abundance observed by the Pioneer Venus mass spectrometer, no argon emission was detected 
Fig. 1 Average EUV dayglow spectrum obtained with the UVIS spectrograph during the Cassini flyby of Venus (black line). For comparison, the Mars dayglow spectrum obtained with the Far Ultraviolet Explorer (FUSE) telescope has been plotted at the spectral resolution of the UVIS instrument (red curve). Note the absence of the Ar line at $106.6 \mathrm{~nm}$ in the Venus spectrum (adapted from on Gérard et al. 2011a)

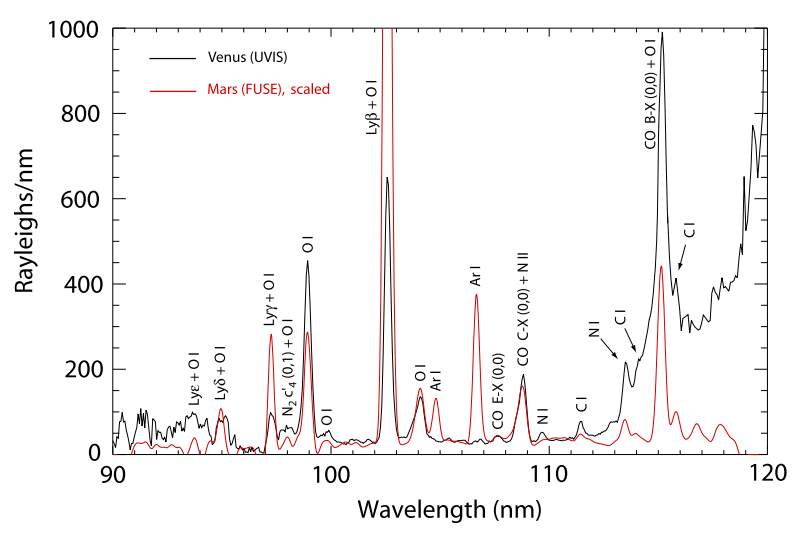

in the Venus spectrum, by contrast to the Mars spectrum. The intensity of the He I line at $58.4 \mathrm{~nm}$ was determined as $\sim 300 \mathrm{R}$, consistent with prior measurements from Venera and Galileo.

EUV spectra at $0.37 \mathrm{~nm}$ resolution obtained with the UVIS spectrograph on board Cassini during the Venus flyby on June 24, 1999 were analyzed by Gérard et al. (2011a) (Fig. 1). The spectral range of the instrument extended from 80 to $190 \mathrm{~nm}$. The projection of the UVIS slit scanned the Venus sunlit disk at low latitudes at a phase angle close to $99^{\circ}$. The solar activity level was high $\left(\mathrm{F}_{10.7}\right.$ at Earth distance $\left.=214\right)$. It was shown that the observed intensity of the OII emission at $83.4 \mathrm{~nm}$ is higher than predicted by the airglow model described by Gérard et al. (2008a), but that an increase of the $\mathrm{O}^{+}$ion density relative to that usually measured by Pioneer Venus could bring the observations and the modeled values into better agreement. The intensity of the OI $98.9 \mathrm{~nm}$ multiplet was correctly predicted if the previously neglected resonance scattering of solar radiation by $\mathrm{O}$ atoms was included as a source. The presence of the $(0,0) \mathrm{C}-\mathrm{X} \mathrm{CO}$ band was confirmed. The calculated intensity of the $\mathrm{CO} \mathrm{B}-\mathrm{X}$ emission along the track of the UVIS slit was also in fair agreement with the observations. The brightness of the NI $120 \mathrm{~nm}$ multiplet exceeded the observed values by a factor of $\sim 2-3$ but the difference was reduced to $30-80 \%$ if the electron impact on and photodissociation of $\mathrm{N}_{2}$ sources of $\mathrm{N}$ atoms are considered as optically thin. The authors concluded that the empirical VTS3 model (Hedin et al. 1983) provides $\mathrm{O}, \mathrm{N}_{2}$ and CO densities leading to satisfactory agreement between the calculated and the observed EUV airglow emissions.

Using the Extreme Ultraviolet Spectroscope for Exospheric Dynamics (EXCEED) on board Hisaki, Masunaga et al. (2015) observed the variations of the global brightness of the OII $83.4 \mathrm{~nm}, 130.4 \mathrm{~nm}$ and $135.6 \mathrm{~nm}$ dayglow emissions during three quasi-continuous time intervals in 2014. The intensities were somewhat higher than the UVIS values discussed here. Several periodicities were identified in addition to the 29-day modulation caused by the solar rotation effects on the solar EUV input (see Sect. 4.2.1). Additionally, Masunaga et al. (2017) pointed out the presence of a 4-day periodicity of the OI $135.6 \mathrm{~nm}$ dayglow brightness that was dominant on the dawn side of the planet. A proposed mechanism is a 4-day asymmetric modulation of the eddy diffusion produced by 4-day periodic variations of the wind velocity of the super-rotating middle atmosphere, in the presence of upward propagating gravity waves. As a consequence, the $\mathrm{O}$ density and thus the $135.6 \mathrm{~nm}$ airglow presumably exhibit the observed periodicity. Whatever the details of the involved physical processes, the authors suggest that the $\sim 4$-day periodicity of the dayglow variations reflects the dynamics of the super-rotating atmosphere. 
Fig. 2 Variation of the HeI $58.4 \mathrm{~nm}$ airglow intensity along the UVIS foot track on the Venus disk (diamonds). The solid line shows the modeled intensity with the helium density from the VTS3 model and the solar flux from the EUVAC solar flux model. The dotted lines show the modeled intensities calculated by scaling the helium density by factors equal to $1 / \mathrm{e}, 0.5,2$ and $\mathrm{e}$. The dashed line represents the calculated intensity distribution with the solar flux from Woods and Rottman (2002) (from Gérard et al. 2011b)

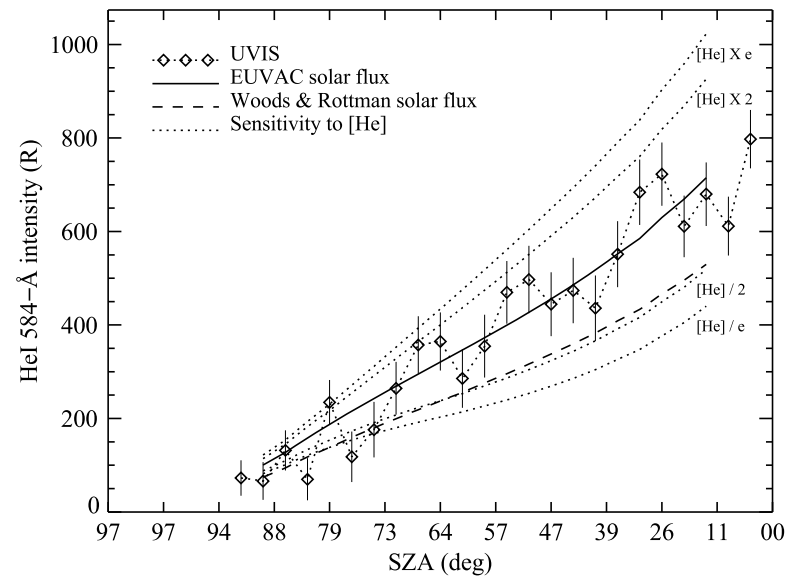

Observations of the He I resonance line at $58.4 \mathrm{~nm}$ made with the Extreme Ultraviolet Explorer Satellite (EUVE) in April 1998 provided a mean value of $144 \pm 32$ R (Krasnopolsky and Gladstone 2005). The corresponding helium mixing ratio of $9 \pm 6 \mathrm{ppm}$ in the lower and middle atmosphere is in agreement with earlier in situ and remote sensing abundance determinations. They argued that the loss of helium is compensated by outgassing of helium produced by radioactive decay of uranium and thorium and by capture of the solar wind $\alpha$-particles with an efficiency of 0.1 . The surprisingly bright emission of $\mathrm{He}^{+}$at $30.4 \mathrm{~nm}$ suggested that charge exchange in the flow of the solar wind $\alpha$-particles around the Venus ionopause is much stronger than in the flow of $\alpha$-particles into the ionosphere. The brightness of the He $58.4 \mathrm{~nm}$ resonance line was also measured along the Venus disk during the UVIS flyby (Fig. 2). The mean disk intensity was $\sim 320 \mathrm{R}$ and the bright limb reached $\sim 700 \mathrm{R}$, slightly higher than those determined from previous observations. The intensity distribution along the UVIS footprint on the disk was best reproduced using the EUVAC solar flux (Richards et al. 1994) together with the helium density distribution from the VTS3 empirical model. A dayside helium density close to $8 \pm 4 \times 10^{6} \mathrm{~cm}^{-3}$ at the altitude where the $\mathrm{CO}_{2}$ density is $2 \times 10^{10} \mathrm{~cm}^{-3}(\sim 145 \mathrm{~km})$ was derived from the UVIS-Cassini disk observations. This value compares reasonably well with earlier determinations of $2 \pm 1 \times 10^{6} \mathrm{~cm}^{-3}$ from Mariner 10 (Kumar and Broadfoot 1975), revised to $1.8 \pm 0.9 \times 10^{6} \mathrm{~cm}^{-3}$ by Krasnopolsky and Gladstone (2005), $2.6 \pm 1.2 \times 10^{6} \mathrm{~cm}^{-3}$ from Venera 11 and 12 (Chassefiere et al. 1986), $6.8 \times 10^{6} \mathrm{~cm}^{-3}$ from the Pioneer Venus bus descent (von Zahn et al. 1983), $4.3 \pm 1.9 \times 10^{6} \mathrm{~cm}^{-3}$ from EUV Explorer $\left(4.3 \pm 1.9 \times 10^{6} \mathrm{~cm}^{-3}\right.$ from EUV Explorer (Krasnopolsky and Gladstone 2005), and $8 \times 10^{6} \mathrm{~cm}^{-3}$ from the VTS3 model on the dayside. Airglow observations have confirmed that measurements of the HeI $58.4 \mathrm{~nm}$ emission may be used to determine the helium density distribution in the Venus upper atmosphere. The helium escape rate at the top of the atmosphere was estimated $4.4 \times 10^{24}$ atoms s $^{-1}$ by Krasnopolsky and Gladstone (2005).

\subsection{Far Ultraviolet}

Hubert et al. (2010) analyzed FUV spatially resolved dayglow spectra between 111.5 and $191.2 \mathrm{~nm}$ obtained at $0.37 \mathrm{~nm}$ resolution with UVIS during the Cassini flyby (Fig. 3). They concentrated on the OI 130.4 triplet and $135.6 \mathrm{~nm}$ doublet and the CO A-X Fourth Positive bands. They compared the brightness observed along the UVIS foot track of the two OI 


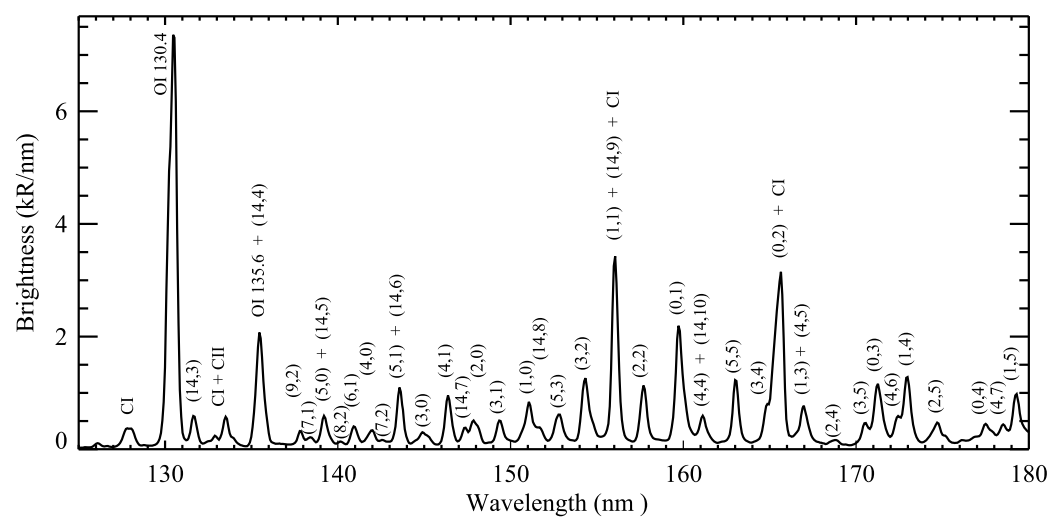

Fig. 3 Average FUV dayglow spectrum measured during the Cassini flyby of Venus. The identified molecular bands belong to the CO Fourth Positive system (from Hubert et al. 2010)

multiplets with that deduced from an airglow model where the neutral atmospheric densities were taken from the VTS3 model by Hedin et al. (1983). Using the EUV solar intensities appropriate to the time of the observation, the modeled intensities agreed with the observed $130.4 \mathrm{~nm}$ brightness within $\sim 10 \%$. The OI $135.6 \mathrm{~nm}$ brightness was also reasonably well reproduced by the model. It was also found that self-absorption of the $\left(0-v^{\prime \prime}\right)$ bands of the CO Fourth Positive system is important. The derived CO vertical column of $6.4 \times 10^{15} \mathrm{~cm}^{-2}$ was in close agreement with the value of $8.7 \times 10^{15} \mathrm{~cm}^{-2}$ given by the VTS 3 model.

The brightness of the ultraviolet multiplets of neutral carbon at $126.1,156.1$ and $165.7 \mathrm{~nm}$ were determined using along the Cassini track by Hubert et al. (2012). The intensities were compared with the results of a full radiative transfer model of these emissions including the known photochemical sources of photons and resonant scattering of sunlight. The carbon density profile of the Venus thermosphere had never been directly measured. The authors found that intensity of the carbon multiplets were substantially larger than the modeled values using a $C$ density profile taken from the model by Fox and Paxton (2005), driven by observations from OUVS on board Pioneer Venus. They applied a scaling factor increasing monotonically with solar zenith angle, ranging from less than 1 to more than 20 to the carbon column. They suggested that this discrepancy possibly originates from the photochemistry of molecular oxygen to which the carbon density is highly sensitive. This point will be further discussed in Sect. 3.5.

Chaufray et al. (2012a) presented the first limb observations of the CO Cameron bands between 180-260 nm and $\mathrm{CO}_{2}^{+}$doublet at $289 \mathrm{~nm}$. They were obtained with the wide portion of the SPICAV instrument on board Venus Express. The Cameron bands brightness peaks at $137.5 \pm 1.5 \mathrm{~km}$ with a brightness of $2000 \pm 100 \mathrm{kR}$ and the $\mathrm{CO}_{2}^{+}$doublet is maximum at $135.5 \pm 2.5 \mathrm{~km}$ with a peak brightness of $270 \pm 20 \mathrm{kR}$ (Fig. 4). The temperature near $145 \mathrm{~km}$ derived from the $\mathrm{CO}_{2}^{+}$bands scale height is $290 \pm 60 \mathrm{~K}$. The spectral shape of the Cameron bands is similar to that observed on Mars at the same coarse 10-nm resolution. The stronger brightness of the Venusian dayglow with respect to the Mars dayglow in the 200-300 nm range cannot only be explained by the distance to the Sun and by the difference in EUV solar flux at the time of the observations. Chaufray et al. (2012a) argued that the larger abundance of carbon monoxide in the Venus upper atmosphere provides an additional intensity contribution through the $\mathrm{e}+\mathrm{CO} \rightarrow \mathrm{CO}\left(\mathrm{A}^{1} \Pi\right)+$ e excitation process, compared to Mars where this process is considered negligible. This possibility and the uncertainties on the excitation cross sections were examined by Bhardwaj and Jain (2013). 

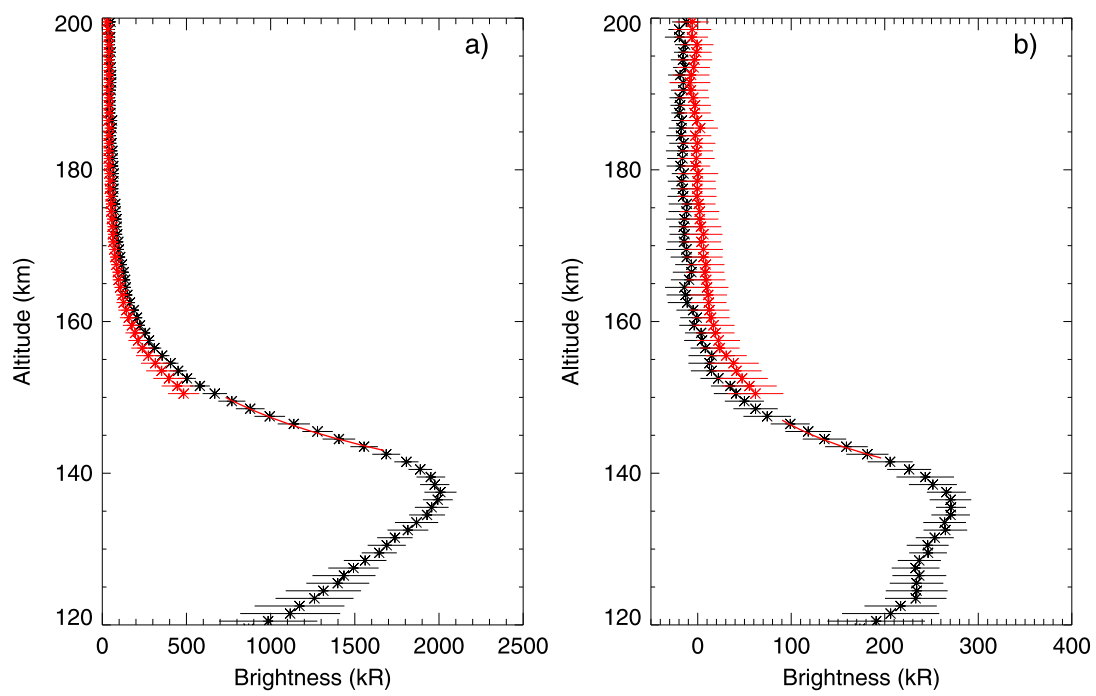

Fig. 4 Limb profile of (a) the Cameron bands and (b) $\mathrm{CO}_{2}^{+}(\mathrm{B}-\mathrm{X})$ bands brightness. The vertical scale corresponds to the altitude of the tangent point. The observed signal is saturated below $150 \mathrm{~km}$ with the binning mode (in red) (from Chaufray et al. 2012a)

The $(0,5)$ and $(0,6)$ bands of the $\mathrm{N}_{2}$ Vegard-Kaplan band system have been identified on Mars with the SPICAM-UV spectrometer by Leblanc et al. (2007). Their presence was confirmed and the $\mathrm{N}_{2}$ LBH bands were also detected with MAVEN/IUVS (Stevens et al. 2015) but no $\mathrm{N}_{2}$ emission has been observed so far on Venus. Fox and Hać (2013) predicted the overhead intensities of $17 \mathrm{~N}_{2}$ band systems and limb profiles of the Vegard-Kaplan bands. They expected that the Venus $\mathrm{N}_{2}$ emissions are 5.5-9.5 times stronger than those of Mars, as the $\mathrm{N}_{2}$ mixing ratio is larger in the Venus thermosphere and because the solar flux is about four times greater at the orbit of Venus than that at Mars. Bhardwaj and Jain (2012) calculated $\mathrm{N}_{2}$ triplet state intensities on Venus about a factor 10 higher than those on Mars. The lack of detection of $\mathrm{N}_{2}$ features in the Venus UV spectrum remains currently unexplained.

\subsection{Exospheric O and H Airglow}

Observations of the Lyman- $\alpha$ dayglow from the Venusian hydrogen corona made by SPICAV on Venus Express have been analyzed by Chaufray et al. (2012b) to estimate the amount of hydrogen in the Venus exosphere. The Lyman- $\alpha$ brightness profiles derived were reproduced by the sum of a cold hydrogen population dominant below $\sim 2000 \mathrm{~km}$ and a hot hydrogen population dominant above $\sim 4000 \mathrm{~km}$. The exospheric atom distribution and the implications on the atom escape flux will be considered in detail in Sect. 6. No observation from the SPICAV spectrograph establishing the presence of hot oxygen atoms in the Venus exosphere has been published so far. The reader is also referred to the review of evidence from earlier observations by Fox and Bougher (1991) and to the discussion of modeling results by Futaana et al. (2011).

\subsection{Nitric Oxide Nightglow}

Radiative two-body recombination of $\mathrm{N}\left({ }^{4} \mathrm{~S}\right)$ and $\mathrm{O}\left({ }^{3} \mathrm{P}\right)$ ground-state atoms produces chemiluminescent emission of the nitric oxide $\gamma\left(\mathrm{A}^{2} \Sigma-\mathrm{X}^{2} \Pi\right)$ and $\delta\left(\mathrm{C}^{2} \Pi-\mathrm{X}^{2} \Pi\right)$ bands. In this 
process and subsequent radiative cascades only the $v^{\prime}=0$ vibrational levels of the A and $\mathrm{C}$ states are efficiently produced. The structure and distribution of the nightside airglow was investigated using the PV-OUVS spectrometer on board Pioneer-Venus (Stewart et al. 1980). Extensive observations in the limb and nadir directions were initially performed with the SPICAV spectrograph. The altitude distribution of the emission rate and its variability have been analyzed by Stiepen et al. (2012).

The global statistical distribution of the nadir intensity was mapped and confirmed the shift of the bright statistical region by 2-3 hours toward dawn, slightly south of the equator. Unlike PV-OUVS that provided NO airglow spin-scan images every 24 hours, the SPICAV spectrograph (Bertaux et al. 2007) did not generate global images of the Venus nightside. It provided spectra at 1.5 or $5 \mathrm{~nm}$ resolution in the limb or nadir direction. Statistical maps of the NO nightglow were constructed by co-adding measurements of the total NO intensity measured along the nadir line of sight (Stiepen et al. 2013). Limb observations were compared with 1-D model outputs (Gérard et al. 2008b; Stiepen et al. 2013) to determine the magnitude of the eddy diffusion coefficient near the homopause. The signature of the $\mathrm{C}\left({ }^{2} \Pi\right) \rightarrow \mathrm{A}\left({ }^{2} \Sigma\right)$ NO transition at $1.224 \mu \mathrm{m}$ was unambiguously detected with VIRTIS in the Venus nightglow on two occasions by García-Muñoz et al. (2009a). This transition populates the A upper state of the $\gamma$ bands observed with SPICAM. A branching ratio of 0.32 between the $\mathrm{C} \rightarrow \mathrm{A}$ and the $\mathrm{C} \rightarrow \mathrm{X}$ transitions was determined from the comparison with the brightness of the FUV NO emission, a value mid-way in the range of earlier theoretical and experimental determinations.

\subsection{Visible Nightglow}

Observations of the molecular oxygen visible airglow on the Venus nightside made with the visible channel of the VIRTIS spectral imager have been reported by Garcia-Muñoz et al. (2009b) and Migliorini et al. (2013a). They identified the presence of several $\left(0-v^{\prime \prime}\right) \mathrm{O}_{2}$ Herzberg II $\left(\mathrm{c}^{1} \Sigma_{\mathrm{u}}-\mathrm{X}^{3} \Sigma_{\mathrm{g}}\right)$ bands and the (0-6), (0-7) and (0-8) bands of the $\left(\mathrm{A}^{\prime 3} \Delta_{\mathrm{u}}-\mathrm{a}^{1} \Delta_{\mathrm{g}}\right)$ Chamberlain bands (Fig. 5). These features were first observed from the Venera 9 and 10 spacecraft by Krasnopolsky (1983). Migliorini et al. (2013a) obtained limb profiles of the observed bands and relative intensities from VIRTIS limb observations. The wavelengthintegrated intensities of the Herzberg II bands, with $v^{\prime \prime}=7-11$, were inferred from the observed spectra. The limb intensity is in the range $84-116 \mathrm{kR}$ at the altitude of the peak

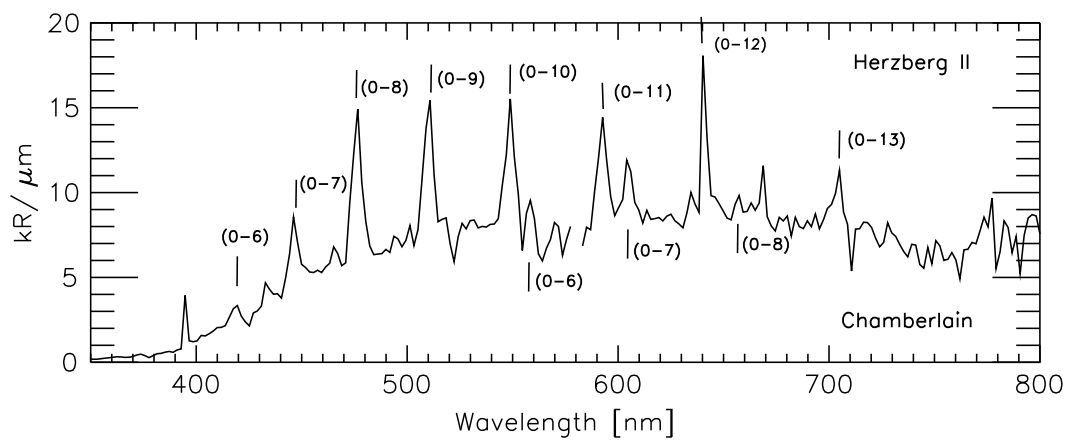

Fig. 5 Average nightglow spectrum observed with VIRTIS in the 350-800 nm spectral range. The identified Herzberg II and Chamberlain transitions are marked by the $\left(0-v^{\prime \prime}\right)$ labels above and below the spectral lines, respectively (from Migliorini et al. 2013a) 
at 93-98 km. For the first time, the $\mathrm{O}_{2} \mathrm{a}^{1} \Delta_{\mathrm{g}}-\mathrm{X}^{3} \sum$ and the $\mathrm{O}_{2}$ Herzberg II emission were simultaneously investigated with VIRTIS-M. The peak altitude of the $\mathrm{O}_{2}$ Chamberlain bands was found about $4 \mathrm{~km}$ higher that the Herzberg II bands. These observations led Gérard et al. (2013) to model the vertical distribution of these emissions and to re-examine the quenching coefficients of the $\mathrm{c}^{1} \Sigma_{\mathrm{u}}$ and $\mathrm{A}^{\prime 3} \Delta_{\mathrm{u}} \mathrm{O}_{2}$ levels by $\mathrm{CO}_{2}$ and $\mathrm{O}$. The visible $\mathrm{O}_{2}$ nightglow was also imaged with the Venus Monitoring Camera (VMC). García-Muñoz et al. (2013) presented conclusions in agreement with the spectral VIRTIS spectral images.

The $\mathrm{O}\left({ }^{1} \mathrm{~S} \rightarrow{ }^{1} \mathrm{D}\right)$ green line at $557.7 \mathrm{~nm}$ was initially detected in the Venus nightglow by Slanger et al. (2001) with the HIRES spectrograph on the Keck I 10-m telescope. They measured a brightness $\sim 150 \mathrm{R}$. The $\mathrm{O}\left({ }^{1} \mathrm{D} \rightarrow{ }^{3} \mathrm{P}\right)$ red line at $630.0 \mathrm{~nm}$ was not observed. The spectra also showed $\sim 700 \mathrm{R}$ of Herzberg II bands and $\sim 120 \mathrm{R}$ of Chamberlain bands. The oxygen green line was absent in the same spectral region in 1975 in the Venera 9 and 10 observations (Krasnopolsky 1983). Subsequent observations confirmed the presence of the green line, but the intensity was very variable (Slanger et al. 2006, 2012), including periods when it was not discernible. This strong variability was interpreted as a signature of a strong control by solar activity, suggesting a possible ionospheric origin of the emission (Slanger et al. 2012). Fox (2012) noted that if the oxygen green line has an ionospheric source, the energy of the precipitating electrons should be high enough to allow them to penetrate below $150 \mathrm{~km}$, so that the green line is excited but the $630.0 \mathrm{~nm}$ red line is collisionally quenched. The green line was detected again for the first time since 2004 by Gray et al. (2014) who suggested that energetic particle precipitation is the main contributor to the nightside $557.7 \mathrm{~nm}$ emission (see Sect. 2.9).

\subsection{Infrared Dayglow}

\subsubsection{Solar Fluorescence and Non-LTE Modeling}

The interest in the infrared part of the Venus dayglow was largely triggered by the discovery from ground based telescopes in the seventies of a strong emission at $10 \mu \mathrm{m}$ in the upper atmospheres of both Venus and Mars (Johnson et al. 1976; Mumma et al. 1981). In contrast to the UV airglow, direct solar absorption in the IR part of the spectrum usually excites ro-vibrational states in the ground electronic state of the atmospheric species. Cascading of the initial excited state complicates the analysis because of the competition between radiative relaxations in several possible transitions and collisional quenching. Theoretical models of the key $\mathrm{CO}_{2}$ vibrational states were specifically developed to explain this 10- $\mu \mathrm{m}$ emission (Deming et al. 1983; Gordiets and Panchenko 1983). Later developments ended up with more generic models able to explain the $10-\mu \mathrm{m}$ emission but also included many more $\mathrm{CO}_{2}$ and $\mathrm{CO}$ infrared bands and vibrational levels, all of them in competition with exchanges of vibrational energy during vibrationaltranslational and vibrational-vibrational collisional processes (Stepanova and Shved 1985; Roldan et al. 2000). For a detailed discussion of non-LTE models in the IR in planetary atmospheres, the reader is referred to the work by López-Puertas and Taylor (2001). The non-local thermodynamic equilibrium models (non-LTE) are handy tools to analyze many

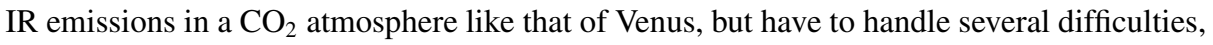
normally with diverse approximations and iterations. One of the difficulties is the uncertainty in collisional rates among many states, specially isotopic and high energy states. Another one is that strong radiative transfer among multiple layers in thick atmospheres with varying composition and temperature require detailed calculations in many ro-vibrational bands. In addition there is the inherently non-linear problem of radiation-matter interaction: 

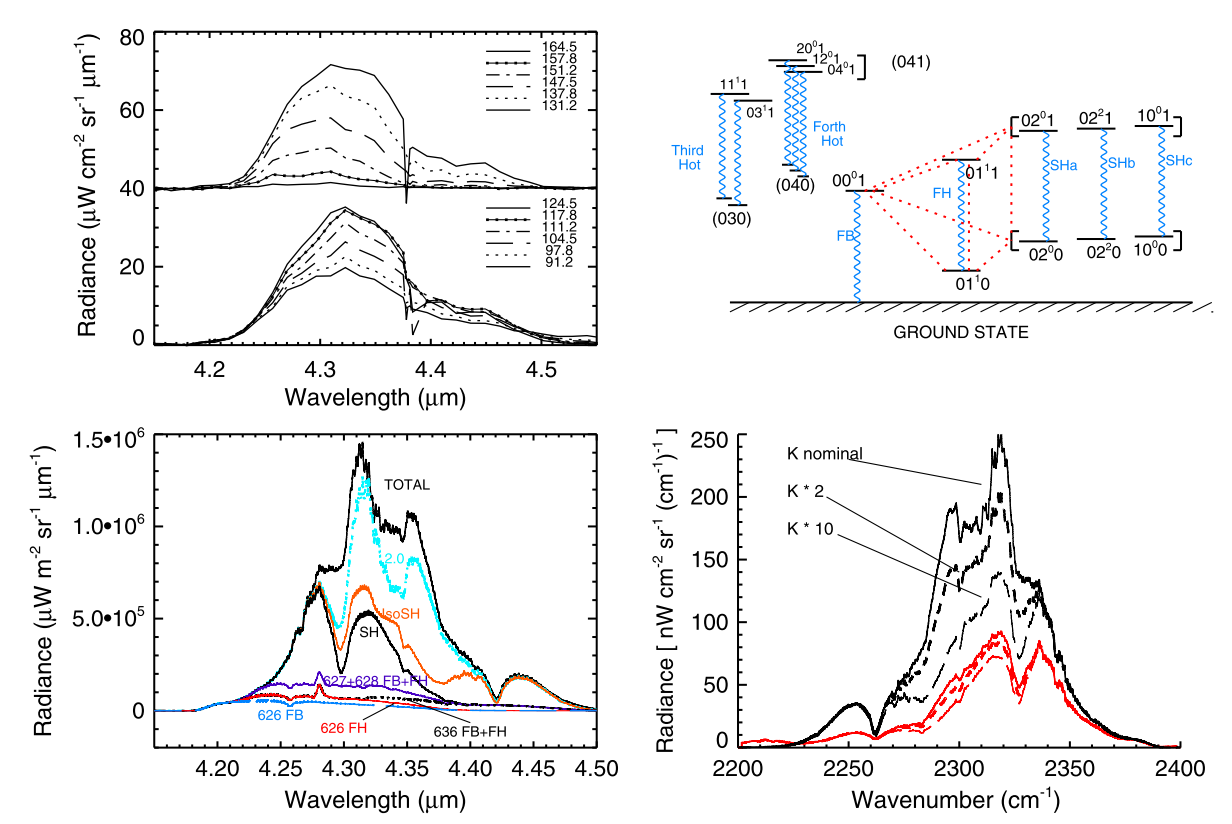

Fig. 6 Measurements of Venus in the $4.3 \mu \mathrm{m}$ region taken by NIMS/Galileo, at different tangent altitudes, indicated in $\mathrm{km}$. Altitudes higher than $125 \mathrm{~km}$ are shifted by $4 \times 105$ radiance units for clarity. Top-right: schematic diagram of $\mathrm{CO}_{2}$ levels included in the non-LTE model used to explain the data; names of bands (in blue), collisional exchanges (in red) and states follow López-Valverde et al. (2007). Bottom-left: contributions from different $\mathrm{CO}_{2}$ IR bands to the total simulated limb emission at a tangent altitude of $110 \mathrm{~km}$; band names are as in the top-right panel. Bottom-right: effect of increases of the collisional V-V relaxation of high energy states by factors 2 and 10 on the emitted limb spectra for two different reference atmospheres (black and red) with different densities at $110 \mathrm{~km}$ (from López-Valverde et al. 2011a). See text

radiative heating and cooling rates, which are dependent on the thermal state, affect such a state. Many of these model predictions remain untested in the case of Venus due to the lack of systematic observations in the IR until recently. One space experiment in the pre-Venus Express era which measured the spectral and altitude shape of the strong daytime infrared emissions of $\mathrm{CO}_{2}$ at $4.3 \mu \mathrm{m}$ was the NIMS instrument on board the Galileo mission. During the Galileo flyby of Venus in 1993 NIMS acquired the first atmospheric IR spectra of our neighbor planet in a limb viewing geometry at these wavelengths (Fig. 6, upper left panel). The spectra, at a low-moderate resolution showed a single-broad-maximum emission centered at $4.32 \mu \mathrm{m}$ which remained inexplicable until a decade later, when López-Valverde et al. (2007) revised and extended the non-LTE model of Roldan et al. (2000) with many more levels and bands. A large number of hot and combination bands contribute to the limb emission, whose convolution produces the broad feature centered at $4.32 \mu \mathrm{m}$ (Fig. 6, bottom-left panel). Fig. NLTE-1 Top-left: L.

Another feature in the NIMS spectra that appeared surprising in view of the model results, and later confirmed with VIRTIS/Venus Express measurements, is the large emission observed around $4.45 \mu \mathrm{m}$ (or $2250 \mathrm{~cm}^{-1}$ ). Not many $\mathrm{CO}_{2}$ bands contribute there, except for some isotopic (636) bands. The broad and weak emission around $4.45 \mu \mathrm{m}$ is also present in Martian spectra obtained by PFS and OMEGA. López-Valverde et al. (2011a) proposed a revision of some uncertain rate coefficients in current non-LTE models, like the vibrationalto-vibrational relaxation of high energy states. This rate is uncertain by at least a factor 2 or 3 
and they proposed to increase their value. The effect is to increase the quenching and reduce the radiative relaxation in the hot bands that produce the central emission. This solution has the merit to make the relative importance of the isotopic bands around $4.45 \mu \mathrm{m}\left(2247 \mathrm{~cm}^{-1}\right)$ larger in both planets, as shown for the case of Venus in Fig. 6 (bottom-right panel).

\subsubsection{Recent Observations of $\mathrm{CO}_{2}$ Infrared Emissions}

The more recent and extensive observations of $\mathrm{CO}_{2}$ fluorescence in the IR are those of VIRTIS on board Venus Express. However, observations from the ground at very high spectral resolution have also permitted deducing temperatures and winds in the upper mesosphere of Venus. We first review this dataset.

Ground Based Observations at $10 \mu \mathrm{m}$ Heterodyne spectroscopy at $10 \mu \mathrm{m}$ continues to be useful to explore the atmospheres of our neighbor planets Venus and Mars. This technique offers the possibility to achieve spectral resolutions as high as $10^{7}$, separating individual rotational lines of $\mathrm{CO}_{2}$. This resolution permits to resolve individual lines and therefore, in principle, the kinetic temperature can be obtained from the line widths obtained. In practice this is prone to errors in nadir mapping, due to the uncertainty associated to the precise determination of the background absorption (Kaeufl et al. 1984). An additional and complementary strategy, when a suitable number of rotational lines are obtained simultaneously, is to derive the atmospheric temperature from the rotational structure of the band, as was done by Sonnabend et al. (2008). From the Doppler shift of the line cores it is also possible to deduce the atmospheric winds (the component along the line-of-sight). Figure 7 shows a portion of the P-branch of the $\mathrm{CO}_{2}$ band at $9.4 \mu \mathrm{m}$, with up to 9 rotational transitions. Similar measurements are possible at Mars although their emission strengths are smaller. The figure also shows a model simulation by López-Valverde et al. (2011b) which illustrates how the emission varies a lot with two key observing angles, the solar zenith angle (SZA) and the emission angle. The emission obviously decreases at high SZA as the solar flux is reduced, while it increases with the emission angle (from a pure nadir sounding to a nearly limb tangent view) as the optical path is larger and the lines are not saturated (optically thin regime).
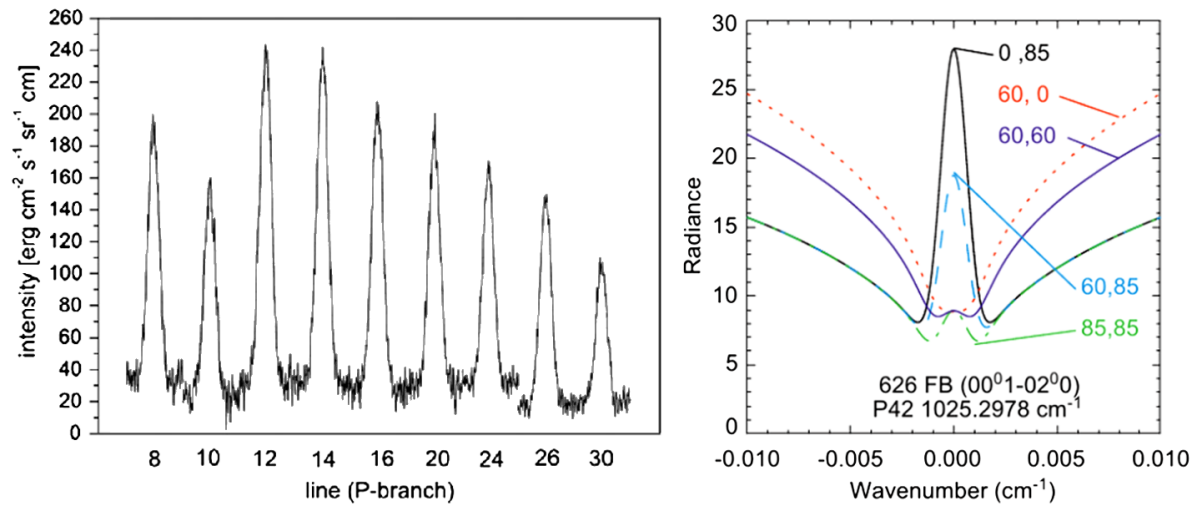

Fig. 7 Left panel: Portion of the P-branch of the $\mathrm{CO}_{2} 9.4$ um band observed at Venus with a resolution better than $1 \mathrm{MHz}$ with HIPWAC at NASA/IRTF in Hawaii (after Sonnabend et al. 2008). Right panel: non-LTE simulation of a typical line ( $\mathrm{P} 42$ of the same $\mathrm{CO}_{2}$ band) showing absorption and emission characteristics varying with the solar zenith angle and with the emission angle; " 0,85 " means "SZA $=0^{\circ}$ and emission angle $=85^{\circ}$ " (after López-Valverde et al. 2011b) 
A major advantage of these ground-based observations is the possibility to perform a global-scale sounding of the planet during a brief campaign, giving an almost instantaneous view of the planetary wind or temperature structure. One disadvantage is that they only permit the sounding of a fixed pressure level in the atmosphere, around $10^{-3} \mathrm{mb}$ (LópezValverde et al. 2011b). This layer is located around $110 \mathrm{~km}$ according to the VIRA daytime reference atmosphere. Caution needs to be taken when interpreting these $10 \mu \mathrm{m}$ data in view of global circulation model predictions, because that fixed pressure layer may vary in altitude with latitude and time (Gilli et al. 2015).

VIRTIS/VEx Observations at $2.7 \mu \mathrm{m}$ and $4.3 \mu \mathrm{m} \quad$ Most of the VIRTIS observations of the $\mathrm{CO}_{2}$ daytime emissions in the IR were made since the beginning of the nominal Venus Express mission in 2006 up to well into the extended mission (2011). VIRTIS is a dual instrument with two components in the infrared: VIRTIS-H, an echelle spectrometer covering the $2-5 \mu \mathrm{m}$ range at a moderate resolution $(\mathrm{R} \sim 1200)$ and VIRTIS-M, a mapping spectrometer from 1 to $5 \mu \mathrm{m}$ at a lower resolution $(\mathrm{R} \sim 200)$. Due to its higher spectral resolution, most analyses of the non-LTE $\mathrm{CO}_{2}$ emissions have focused on VIRTIS-H data so far (Gilli et al. 2015). Sampling of the vertical limb emission with VIRTIS-H was performed mostly at random, with a few points per orbit. During a limited number of orbits, inertial pointing was performed near pericenter, so that vertical sampling was close to $1 \mathrm{~km}$, although at large horizontal displacements. VIRTIS-M maps on the other hand, permitted vertical sampling in the limb portion of the image at its pixel-size resolution, which varies significantly depending on the very variable distance from Venus Express to the planet (Gilli et al. 2009). Figure 8 shows diverse mapping of the $\mathrm{CO}_{2} 4.3 \mu \mathrm{m}$ emission during daytime from VIRTISM, with focus on the limb part. Around the pericenter the VIRTIS-M "spectral mode" was used, obtaining only one row of spectra instead of a 2-D image (as shown in the bottom-right panel). With a typical noise level around $5000 \mathrm{~mW} \mathrm{~m}^{-2} \mathrm{sr}^{-1} \mu \mathrm{m}^{-1}$, Fig. 8 shows that good vertical profiles are available up to about $150 \mathrm{~km}$ (without averaging) in the center of the $4.3 \mu \mathrm{m}$ emission band during pericenter. The top panel also shows that not all the variations across the Venus disc are related to solar zenith angle changes.

Other emissions from $\mathrm{CO}_{2}$ are also visible in the VIRTIS-H data, in particular those at $2.7 \mu \mathrm{m}$. As predicted by models (López-Valverde et al. 2007), this signal is much weaker and suitable averaging is required to obtain spectra above noise levels. Still, a few vertical profiles were built by Gilli et al. (2009) using both VIRTIS-H and VIRTIS-M data. They peaked near the $0.1 \mu$ bar level (115 km in the VIRA daytime reference atmosphere), in agreement with the model predictions. Originated after solar fluorescence from the same $\mathrm{CO}_{2}$ excited states than some of the $4.3 \mu \mathrm{m}$ atmospheric bands, the 2.7 and $4.3 \mu \mathrm{m}$ daytime emissions have not yet been exploited together, in combination with non-LTE models. This is an interesting (and pending) study in order to derive atmospheric densities in the limb and validate other models and datasets in the interesting region of the Venus mesopause.

\subsubsection{CO Fluorescence at $4.7 \mu m$}

Spaceborne Remote Sensing The CO infrared emission at $4.7 \mu \mathrm{m}$ as observed by VIRTIS has been the subject of an intense analysis due to its simpler physics compared to the $\mathrm{CO}_{2}$ emission at $4.3 \mu \mathrm{m}$ and to the possibility to separate lines and therefore, the rotational structure of the band (Gilli et al. 2009, 2011, 2015). The wider separation of the CO lines permitted the identification of two components in the data, the fundamental $\mathrm{CO}(1-0)$ and the first hot $\mathrm{CO}(2-1)$ bands of the main $\mathrm{CO}$ isotope (FB and $\mathrm{FH}$, respectively). As seen in Fig. 9, other hot or isotopic bands are absent in the VIRTIS-H spectra. Interestingly, the 

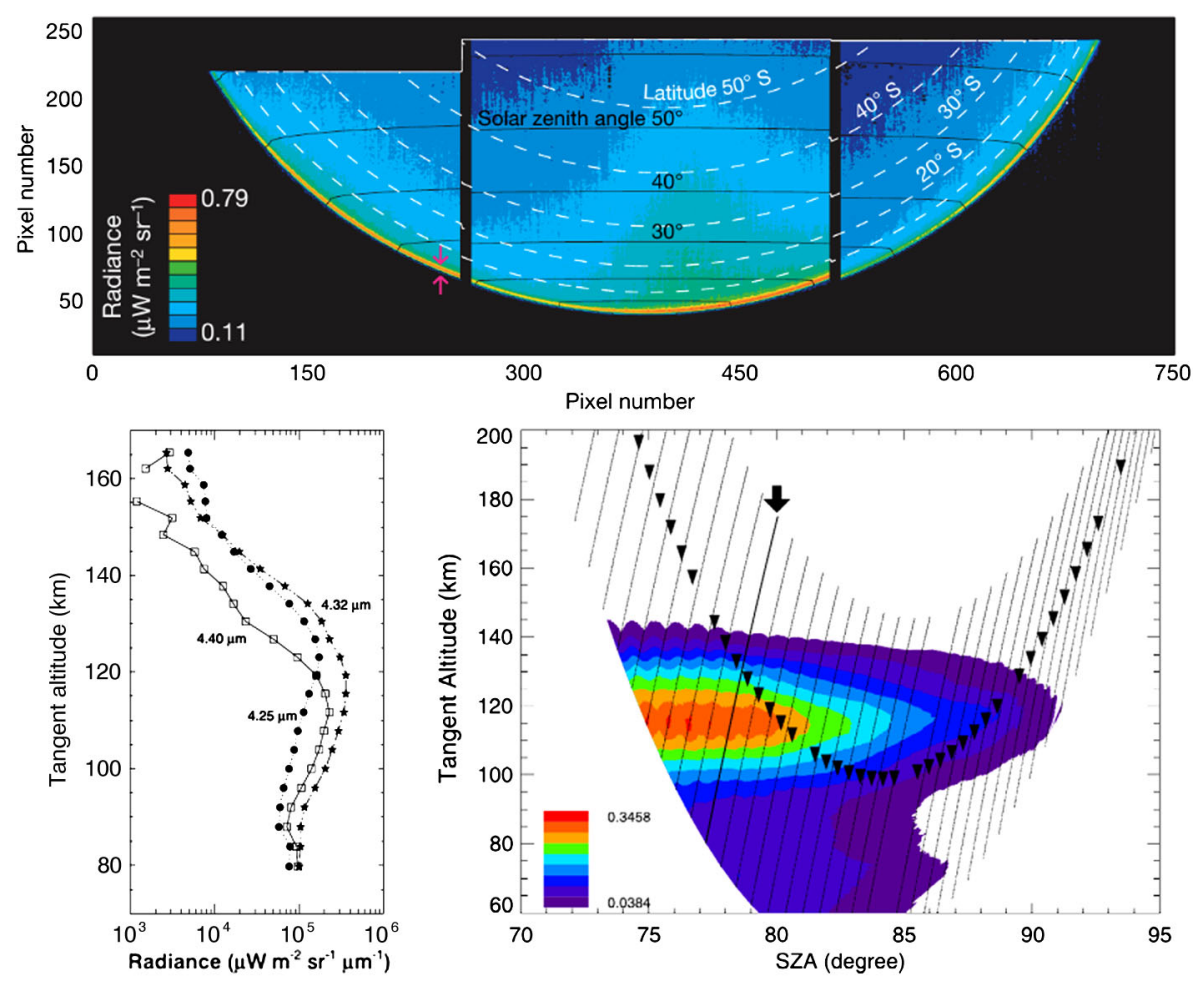

Fig. 8 Top: Composition of three VIRTIS-M spectral data cubes during Orbit 25, at $4.33 \mu \mathrm{m}$, showing nadir and limb emission levels, with variations not totally explained by the expected SZA changes (after Drossart et al. 2007). Latitude circles in red; SZA bands in dashed black lines. Bottom-left: Limb profiles at three wavelengths obtained by combining individual adjacent pixels in one of the cubes of the VIRTIS-M image in orbit 25, in this case with spacing around $4 \mathrm{~km}$ in the vertical. Bottom-right: Set of successive slit-frames of VIRTIS-M from its "spectral mode" during one pericenter observation. Triangles show the center of the slit, where VIRTIS-H data are also available. The emission map only uses VIRTIS-M data (after Gilli et al. 2009)

fundamental and hot band transitions coincide and mix each other in some parts of the spectrum, forming specially strong emission lines, requiring a non-LTE model which couple them properly. This merging can be seen in Fig. 9, with lines P9 (FH) and P15 (FB), at $4.85 \mathrm{~mm}\left(2083 \mathrm{~cm}^{-1}\right)$.

Figure 9 shows an overall good agreement in the altitude and SZA variation between model and data. Due to the sparse VIRTIS-H data coverage in the limb, the study of the altitude variation of the emissions requires the combination of data from many different orbits, either to form vertical profiles or to produce maps like those in Fig. 9. The simulations in this figure, however, correspond to a single atmospheric reference profile. Therefore, the departure from the smooth map of the model's cross-sections possibly represents real atmospheric variability, except at very high SZA where noise includes a significant effect. A proper retrieval scheme is required to exploit this dataset (Gilli et al. 2015). Another interesting result in this map is that, with suitable averages, the radiances present a good behavior up to about $140 \mathrm{~km}$. In the other extreme of the altitude range, the bottom part of the map starts at $95 \mathrm{~km}$. Below this altitude, the radiances at $4.7 \mu \mathrm{m}$ seem to be strongly contaminated by scattering in the Venus high altitude hazes and is not useful for dayglow studies. 
Fig. 9 Top: VIRTIS-H limb spectrum at $4.7 \mu \mathrm{m}$, taken at an altitude around $85 \mathrm{~km}$ and $\mathrm{SZA}=60^{\circ}$, showing individual lines of the $\mathrm{CO}$ fundamental (dash markings) and first hot bands (solid markings) (after Gilli et al. 2009). Middle and Bottom panels: Observations (VIRTIS-H) and model simulations of the altitude/SZA cross section of the $\mathrm{CO}$ emission at $4.7 \mu \mathrm{m}($ at $4.81 \mu \mathrm{m})$; observations combine data from many orbits and become very noisy at high SZA (after Gilli et al. 2015)
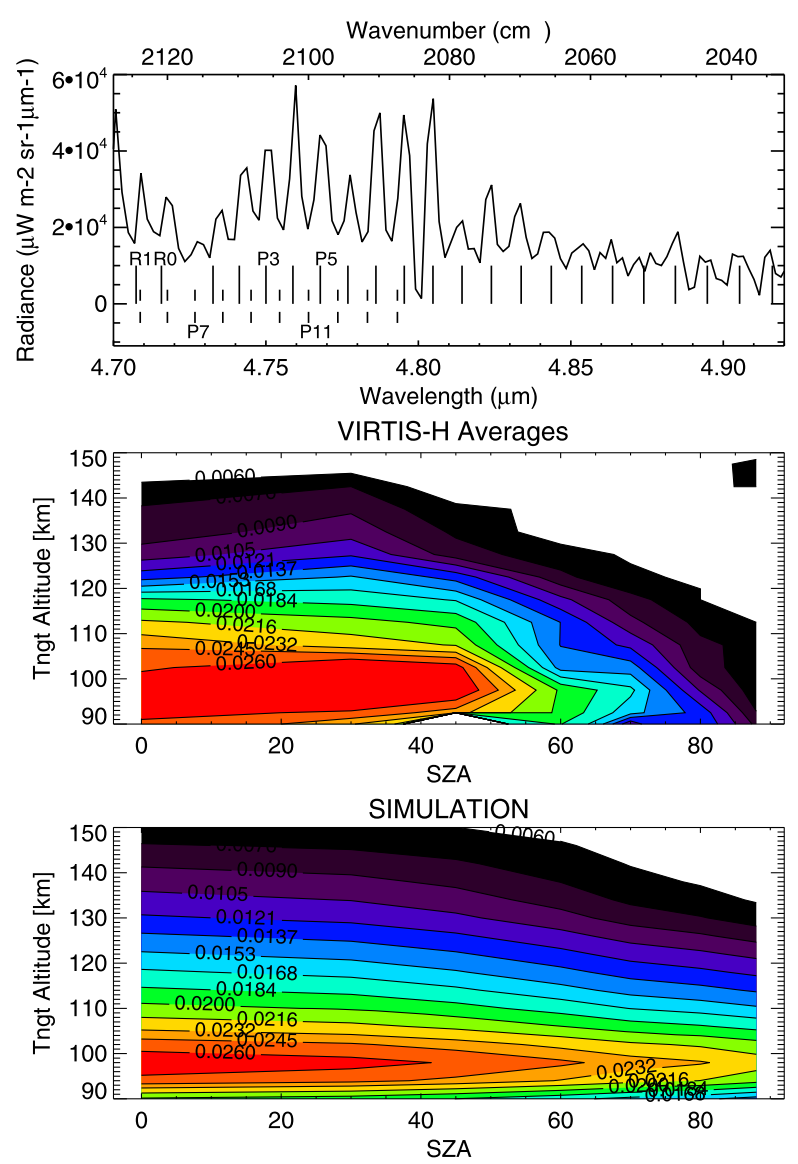

Ground Based/Nadir-Looking Observations at $4.7 \boldsymbol{\mu m}$ As explained by Gilli et al. (2011) observations of CO dayglow from orbit is feasible at the limb but difficult in the nadir with the spectral resolutions and sensitivities available on board Mars Express and Venus Express. Crovisier et al. (2006) and Krasnopolsky (2014a) have demonstrated that this limitation is not present in ground-based observations with sufficiently high resolution. However, the analysis is not simple: the presence of solar and telluric lines requires a precise modeling of the $\mathrm{CO}$ state populations and a careful radiative transfer simulation in order to rely on data-model fittings before extracting key atmospheric parameters.

The first ground-based detection of the $\mathrm{CO}$ fluorescence at $4.7 \mu \mathrm{m}$ was made by Crovisier et al. (2006) using a very high resolution Fourier-transform spectrometer at the CanadaFrance-Hawaii Telescope. They made a detailed analysis of sources of excitation and found the photolysis of $\mathrm{CO}_{2}$ to be an important excitation for the $\mathrm{CO}(v=3)$ and higher states, and after cascading, also for $\mathrm{CO}(v=2)$. Without this source, the common fluorescent selfabsorption at 2.3 and 1.58 um would determine the populations. However, their simulations of the $\mathrm{CO}(2-1)$ first hot band were about a factor 2 too large compared to their data. More recently, Krasnopolsky $(2014 \mathrm{a}, 2014 \mathrm{~b})$ revisited the $\mathrm{CO}(v=2)$ excitation and quenching terms in a couple of works devoted to the observation of $\mathrm{CO}$ dayglow lines on Venus and Mars using the CSHELL spectrograph at the NASA IRTF in Hawaii. He found that the production of $\mathrm{CO}(v=2)$ from $\mathrm{CO}_{2}$ photolysis is lower than assumed before, due to the 
reduced efficiency for photolysis in most of the UV range outside Lyman- $\alpha$, which is a result that seems to explain the excess radiation computed by Crovisier and co-workers.

Krasnopolsky (2014a) observed both the fundamental $\mathrm{CO}(1-0)$ and the first hot $\mathrm{CO}(2-1)$ bands in the dayglow, over a latitude range of $\pm 60^{\circ}$. Six observed lines of the CO dayglow at the hot (2-1) band showed a significant limb brightening typical of an optically thin airglow. Following corrections for the viewing angle and Venus reflection, nadir-looking intensities of the $\mathrm{CO}(2-1)$ band were found constant at about $3.3 \mathrm{MR}$ in the latitude range of $\pm 50^{\circ}$ at a solar zenith angle of $64^{\circ}$. Krasnopolsky (2014a) focused on the $\operatorname{CO}(2-1)$ band, as his best tool to sound the atmospheric temperature at the appropriate emission level, which he found to be around $110 \mathrm{~km}$ for the $2-1$ band. The temperatures found, $204 \pm 10 \mathrm{~K}$, did not present significant latitudinal variations; we review his results further in Sect. 3.1 below. The retrieval of temperature can be done via analysis of the $2-1$ band's rotational distribution, if the spectral resolution is appropriate Let us recall that spectral resolutions on the order of $\mathrm{R} \sim 1000$, like that of VIRTIS-H on Venus Express, are not sufficient to separate lines from the fundamental and first hot bands. This technique can be subject to significant errors if only a few adjacent rotational lines are available. We review his results below in Sect. 3.1. The preference of Krasnopolsky (2014a) for $\mathrm{CO}(2-1)$ over the optically thicker 1-0 fundamental was essentially based on two arguments which are not very strong in our opinion. The first one is the larger guarantee of having rotational LTE at a lower emission layer. In principle this is correct but it seems to be an over-precaution, since rotational LTE is justified and assumed by most Venus NLTE models up to much higher altitudes (Roldan et al. 2000; Crovisier et al. 2006) and therefore, in our opinion should not preclude the fundamental 1-0 band to be used for temperature sounding. The second complication of the 1-0 band, according to Krasnopolsky (2014a), is the presence of saturated emission lines, which alters the rotational distribution of the lines. This is also correct and we agree this is a limitation for the derivation of $\mathrm{CO}$ densities, but it could actually be an advantage for temperature sounding, since the black-body saturated emission will precisely reflect the local kinetic temperature. We recommend this fact to be exploited in future high spectral resolution measurements of this $\mathrm{CO}$ emission band.

\subsection{Infrared Nightside Airglow}

\subsection{1 $\mathrm{O}_{2}\left(\mathrm{a}^{1} \Delta_{\mathrm{g}}\right)$ Nightglow}

A large number of ground-based and VIRTIS observations of the $\mathrm{O}_{2} \mathrm{a}^{1} \Delta_{\mathrm{g}} \rightarrow \mathrm{X}^{3} \sum$ nightglow at $1.27 \mu \mathrm{m}$ were reported with emphasis on different aspects linked to this dipole electric forbidden emission. The spectral resolution of VIRTIS-M spectra did not permit deriving rotational temperatures from the $\mathrm{O}_{2}\left(\mathrm{a}^{1} \Delta_{\mathrm{g}}\right)$ nightglow observations. However, analysis of the VIRTIS-M database (Soret et al. 2012a; Gérard et al. 2014) demonstrated that the altitude of the emission peak is variable and suggested an increasing trend with decreasing solar zenith angle. Abel inversion of the limb observations showed that the volume emission rate is maximum at $97.4 \pm 2.5 \mathrm{~km}$ (Piccioni et al. 2009), while Soret et al. (2012a) found a statistical peak at $99.2 \mathrm{~km}$.

VIRTIS made extensive nadir observations of the southern hemisphere and obtained multiple nightside limb images of the $\mathrm{O}_{2}$ IR airglow at $1.27 \mu \mathrm{m}$. Several studies examined the vertical and horizontal distribution of $\mathrm{O}_{2}\left(\mathrm{a}^{1} \Delta_{\mathrm{g}}\right)$ emission, its variability, relative intensity and structure compared to the $\mathrm{OH}$ and $\mathrm{NO}$ airglow, evidence for gravity waves, atomic oxygen density and implications for the dynamics of the mesosphere-thermosphere transition 
region. These aspects are discussed in Sect. 4. Piccioni et al. (2009) determined from an accumulation of nightglow spectra that the radiance of the (0-1) band at $1.58 \mu \mathrm{m}$ relative to the $(0-0)$ transition was $78 \pm 8$, resulting in a ratio of transition probabilities $\mathrm{A}_{00} / \mathrm{A}_{01}=63 \pm 6$. This value lies within the range of earlier laboratory and airglow determinations.

\subsubsection{OH Nightglow}

Piccioni et al. (2008) discovered the presence of the $\Delta v=1$ and $\Delta v=2$ sequences of the Meinel bands near 1.6 and $3.2 \mu \mathrm{m}$ respectively in the nightglow spectrum (Fig. 10).

The close resemblance to the synthetic spectrum of the $\mathrm{OH}$ Meinel bands was a clear indication that vibrationally excited hydroxyl molecules are present in the upper mesosphere of the Venus nightside. The proposed excitation mechanism was the Bates-Nicolet scheme where ozone molecules reacts with $\mathrm{H}$ atoms to produce vibrationally excited $\mathrm{OH}$ up to $v=9$. The emission peak at the limb is observed between 85 and $110 \mathrm{~km}$ (Gérard et al. 2010; Soret et al. 2010; Migliorini et al. 2011). Krasnopolsky (2010) detected the OH (1-0) P1(4.5) and (2-1) Q1(1.5) airglow lines from the ground in a narrow window using the NASA CSHELL spectrograph. The detected line intensities correspond to (1-0) and (2-1) band intensities of $7.2 \pm 1.8 \mathrm{kR}$ and $<1.4 \mathrm{kR}$ at 21:30 LT and $15.5 \pm 2 \mathrm{kR}$ and $4.7 \pm 1 \mathrm{kR}$ at 4:00 LT. Gérard et al. (2012) described simultaneous VIRTIS spectral images of the OH Meinel and $\mathrm{O}_{2}\left(\mathrm{a}^{1} \Delta_{\mathrm{g}}\right)$ at the limb. They demonstrated that the spatial structure of the two emissions is surprisingly similar and shows a high level of spatial correlation. They suggested that this is a consequence of the role played by $\mathrm{O}$ atoms as a precursor of both emissions. A comparison by Migliorini et al. (2013b) between a VIRTIS spectrum and a high-resolution terrestrial spectrum convolved at a $20-\mathrm{nm}$ resolution showed reasonable agreement. This similarity suggests that the main source of the Meinel bands appears to be the $\mathrm{O}_{3}+\mathrm{H}$ reaction on the two terrestrial planets. Clancy et al. (2013) measured the peak altitude and the brightness of the Meinel bands in the Martian polar winter nightglow with the CRISM instrument. They

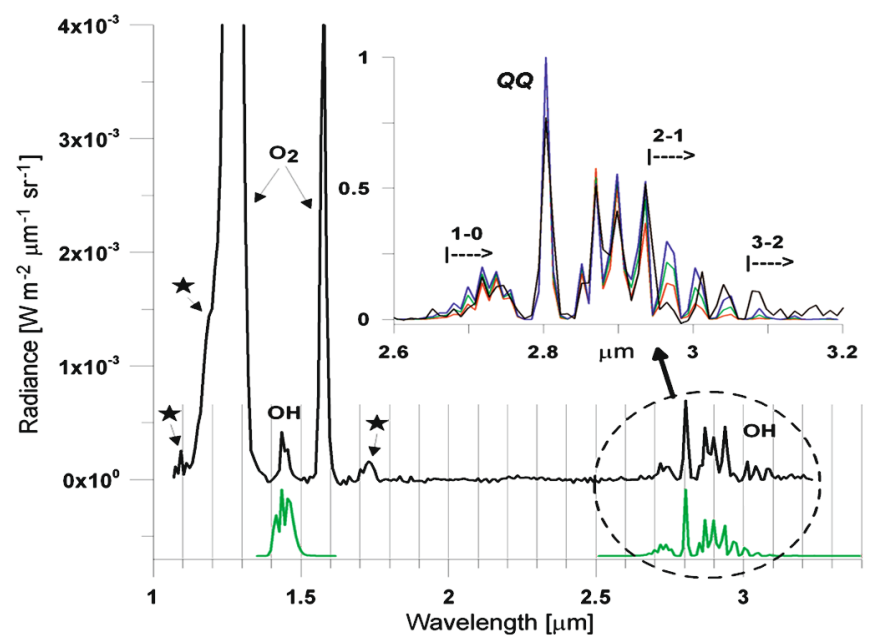

Fig. $10 \mathrm{OH}$ infrared spectrum observed at the limb and comparison with a synthetic spectrum. The two bright features at 1.27 and $1.58 \mu \mathrm{m}$ are the $\mathrm{O}_{2} \mathrm{a}^{1} \Delta \mathrm{g} \rightarrow \mathrm{X}^{3} \Sigma$ bands. In the insert and in green solid line, a comparison is made with a synthetic spectrum of the $\mathrm{OH}$ Meinel bands. The stars indicate the presence of thermal radiation originating from the lower atmosphere (from Piccioni et al. 2008) 
also found that the emission characteristics are consistent with the Bates-Nicolet process as the dominant excitation mechanism. This is an important result as it suggests that, in addition to the direct detection of ozone, the presence of $\mathrm{OH}$ may be used as a bio-signature of the presence of $\mathrm{O}_{3}$ (and therefore $\mathrm{O}_{2}$ ) in exoplanetary atmospheres.

The intensity distribution within the $\Delta v=1$ sequence was used to quantify the role of collisional quenching by $\mathrm{CO}_{2}$ and constrain the abundance of $\mathrm{O}_{3}$ in the upper mesosphere. Soret et al. (2012b) averaged a few thousands observations of the $\mathrm{OH}$ nightglow and obtained a mean peak limb intensity of $350_{-210}^{+350} \mathrm{kR}$ for the $\Delta v=1$ sequence. They identified the (1-0), (2-1), (3-2), and (4-3) bands in the $\Delta v=1$ sequence and determined their respective brightness. They calculated $\mathrm{OH}$ nightglow profiles using both the "sudden death" and single quantum collisional cascade $\Delta v=1$. The "sudden death" model predicts very low intensities in comparison with the observations, while the $\mathrm{OH}$ nightglow intensities were too high in the single quantum model. Consequently, they suggested a reduction of the mean ozone profile from the SPICAV stellar occultations (Montmessin et al. 2011) by a factor of 10 to match the observed $\mathrm{OH}$ nightglow. Krasnopolsky (2013) revised his previous photochemical model for the Venus nighttime atmosphere (Krasnopolsky 2010) to account for the detection of the nighttime ozone layer and the detailed spectroscopy and morphology of the OH nightglow deduced by Soret et al. (2012b) (see Sect. 3.4).

Rotational temperatures in the $95 \mathrm{~km}$ region were derived by comparing the observed and modeled intensity distribution of the $\mathrm{OH}$ Meinel band intensity in the $\Delta v=1$ sequence by Migliorini et al. (2018). They found values ranging from 146 to $198 \mathrm{~K}$, with values of 173,179 and $186 \mathrm{~K}$ for the determinations with an estimated temperature error $\leq 1.5 \mathrm{~K}$. These values are in agreement with most of those determined from various ground based measurements of the $\mathrm{O}_{2}$ IR atmospheric bands (see Sect. 5), as would be expected from the close spatial relationship between the two emissions mentioned before.

\subsection{Aurora}

Highly variable UV emissions had been observed over an 8-year period with PV-OUVS on the nightside (Phillips et al. 1986; Fox and Stewart 1991). They appeared mostly as bright spots of diffuse $130.4 \mathrm{~nm}$ emission, with occasional detections of the OI $135.6 \mathrm{~nm}$ multiplet and CO Cameron bands. The $130.4 \mathrm{~nm}$ intensity varied from less than $\sim 4 \mathrm{R}$ to $20 \mathrm{R}$, with peaks up to $100 \mathrm{R}$. The nightside global mean intensity was $\sim 10 \mathrm{R}$ at solar maximum and $4 \mathrm{R}$ at solar minimum. The dependence on the emission angle did not follow a $1 / \cos$ law and indicated that the emission was optically thick. No clear correlation was found between the brightness and the solar wind parameters, suggesting that the solar wind does not directly modulate the aurora. The CO Cameron bands were also detected with an estimated brightness of $\sim 50 \mathrm{R}$ at the nadir.

Suprathermal electrons have been detected by Knudsen and Miller (1985) above the atmosphere in the Venus umbra by the Pioneer Venus Retarding Potential Analyzer (RPA). These suprathermal electrons belong to two groups. The first one is less energetic and resembles the photoelectron spectra observed in the dayside ionosphere. This population has been interpreted as plasma transported from the day to the nightside. Its characteristic Maxwellian energy is about $7 \mathrm{eV}$. The second (more energetic) group is similar to the population observed in the upper mantle and solar wind near the terminator, with a characteristic energy close to $14 \mathrm{eV}$. Spenner et al. (1996) showed that a suprathermal flux of about 12-eV electrons seems to be present at all altitudes down to at least $200 \mathrm{~km}$. This population is thought to be associated with solar wind plasma making its way into the umbra of Venus, precipitating into the atmosphere and possibly causing the observed UV aurora. 
Fox and Stewart (1991) compared the intensity of the auroral emissions observed by PV-OUVS to that expected from the precipitation of the suprathermal electrons with the reference energy spectrum measured with the RPA on the nightside. Their discrete local loss model produced intensities of $6.4 \mathrm{R}$ for OI $130.4 \mathrm{~nm}, 1.6 \mathrm{R}$ for OI $135.6 \mathrm{~nm}$ and $18 \mathrm{R}$ for $\mathrm{CO}$ Cameron if the 'reference' energy spectrum of super thermal electrons was multiplied by 0.08 . Gérard et al. (2008a) used an electron transport model based on a Monte Carlo implementation of the Boltzmann equation together with a multi-stream radiative transfer model to calculate the effects of multiple scattering on the intensity field of the $130.4 \mathrm{~nm}$ triplet. They showed that the enhancement of the emergent $130.4 \mathrm{~nm}$ intensity by multiple scattering in the optically thick Venus atmosphere increases the auroral $130.4 \mathrm{~nm} / 135.6 \mathrm{~nm}$ ratio by a factor of about 3 , in agreement with the mean ratio observed with PV-OUVS. To account for the average OI auroral emissions, the estimated precipitated energy flux was $2 \times 10^{-3} \mathrm{~mW} \mathrm{~m}^{-2}$.

Fox (2012) suggested that, in the presence of strong electron precipitation, the intensity of the green line on the nightside could intermittently increase by one of several chemical mechanisms, including electron impact ionization of $\mathrm{O}$ or $\mathrm{CO}_{2}$. The ions produced could interact with the neutrals to enhance the production of $\mathrm{O}\left({ }^{1} \mathrm{~S}\right)$ atoms by dissociative recombination. The $\mathrm{O}\left({ }^{1} \mathrm{~S}\right)$ state can also be produced by direct electron impact excitation of ground state $\mathrm{O}$ atoms or electron impact dissociative excitation of $\mathrm{CO}_{2}$ or $\mathrm{CO}$. No detection of auroral emissions has been made so far from Venus Express with SPICAV nor VIRTIS. The $\mathrm{O}^{1} \mathrm{~S} \rightarrow \mathrm{O}^{1} \mathrm{D}$ emission at $557.7 \mathrm{~nm}$, initially believed to be a recombination airglow signature, now appears to be closely related to solar activity. Gray et al. (2014) measured this emission from the ground following solar flares, coronal mass ejections and solar wind streams from December 2010 to July 2012. They found it is highly temporally variable, with the strongest emission $\sim 180 \mathrm{R}$, comparable to the previous brightest detections. They occurred after the three types of solar events, with the strongest emission following CMEs.

\section{Composition and Structure of the Venus Upper Atmosphere}

Although the Venus Express suite of instruments was not specifically oriented toward measurements of neutral species and temperature in the upper atmosphere, significant progress has been made based on airglow observations in the infrared and ultraviolet.

\section{1 $\mathrm{CO}$ and $\mathrm{CO}_{2}$ Densities from Non-LTE IR Emissions}

The derivation of densities from the fluorescence or in general from any non-LTE emission can only be performed with a complex inversion or retrieval process which accounts for the relation between the non-LTE populations and the emerging radiance observed. In other words, it requires using a direct forward model incorporating a precise non-LTE calculation. In addition to the usual difficulties from radiative transfer in many lines and bands, the microscopic processes like collisional energy transfers involved in the calculation of the state populations introduce further uncertainties to the error analysis.

Such a non-LTE inversion process has been completed with success for the CO emission measured by VIRTIS at $4.7 \mu \mathrm{m}$ in the upper mesosphere and in the thermosphere of Venus (Gilli 2012; Gilli et al. 2015). These authors showed that the limb weighting functions in all the CO lines observed are broad, with widths or the order of $20 \mathrm{~km}$, which can be considered as the vertical resolution. Averages of spectra of precisely such size were performed in order to gain SNR. To reduce the noise levels, Gilli et al. (2015) also binned the spectra in latitude 

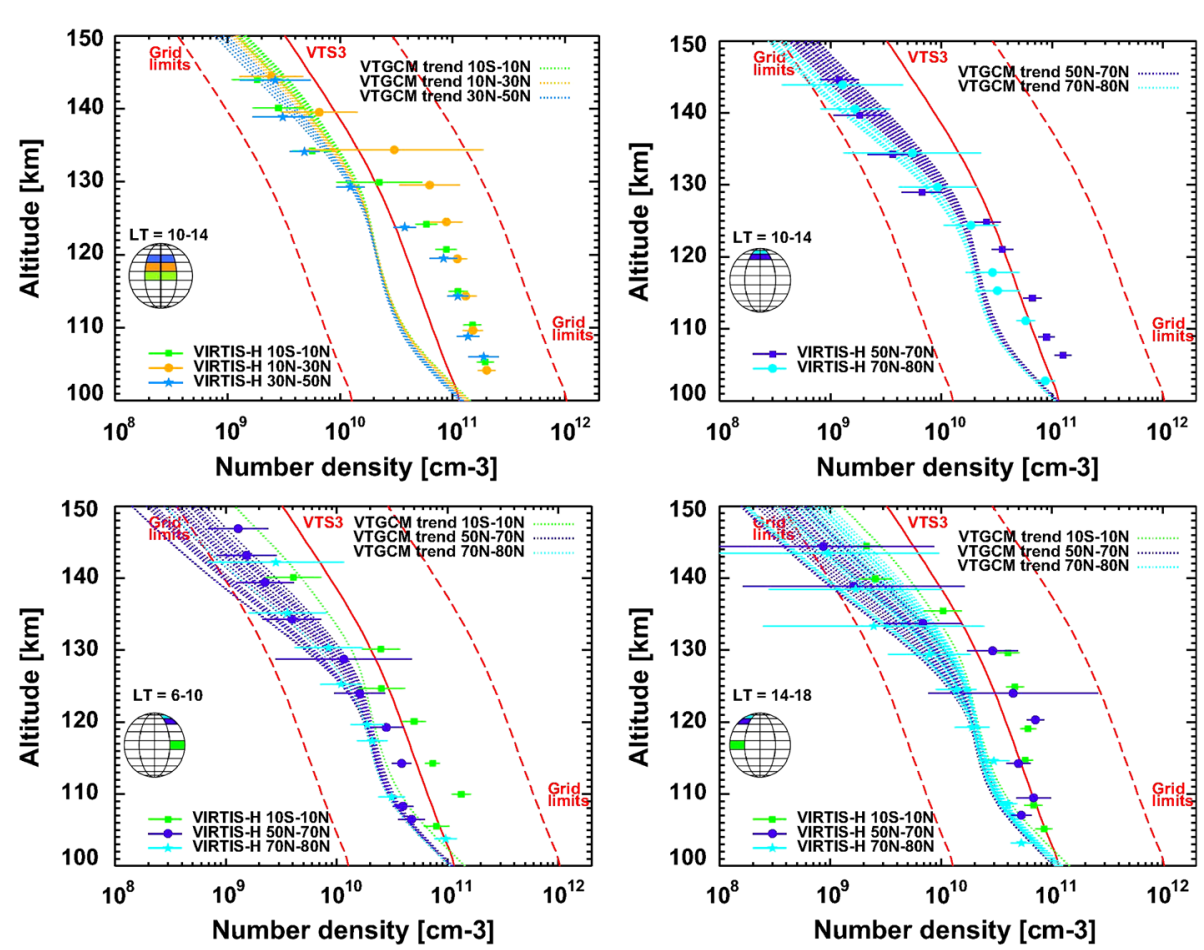

Fig. 11 Selection of retrievals of CO density from Gilli et al. (2015) for different intervals of latitude and local time, as indicated. Top left: three data boxes at low-mid latitudes. Top right: two data boxes at high-latitudes. The two bottom panels represent the same three latitude boxes in the morning (6-10 LT, bottom left panel) and in the afternoon (14-18 LT, bottom right panel). Dashed red lines: limits of the grid of synthetic data used in the retrieval, values beyond them cannot be retrieved. The VTS3 CO density (red solid line) and several VTGCM profiles (averaged for the same latitudinal and local time boxes) are also shown for comparison

ranges of 20 degrees and in three local time intervals. The solar zenith angle (SZA) between $0^{\circ}$ and $90^{\circ}$ was divided in 8 intervals and this parameter was permitted to vary within each of those intervals; the non-LTE retrieval scheme accounts for its effect, i.e., the radiances are largely affected by the SZA, but not the retrieved density. Finally, about 100 successful retrievals of the $\mathrm{CO}$ abundance were obtained, in altitude steps of $5 \mathrm{~km}$, from 100 up to $145 \mathrm{~km}$, as shown in Fig. 11. The latitude coverage is not complete and actually varies with altitude, which limited the analysis of the global distribution of CO. Figure 11 shows that the results have large error bars above about $130 \mathrm{~km}$, where the first hot bands contribution decreases. Other cautions need to be taken in their interpretation. One of them is that the data selection process combines measurements from different orbits, longitudes and illumination conditions in the limb, i.e., the profiles do not form actual 1-D instantaneous vertical views on a given point and at a given moment. This is important for comparison with models and other data sets.

In spite of these difficulties the first global map of CO during daytime in the Venus thermosphere has been obtained and shows interesting features. First, the decrease in altitude is similar to expected 1-D profiles. Second, the data at high solar illumination (LT 10-14 h) show a decrease of abundance from equatorial to high latitudes, of about a factor 2 . This is correct below about $130 \mathrm{~km}$, but above this altitude the variation is smaller or within 
the large noise levels there. This is interesting because GCM models predict a variation but only in the thermosphere, not below about $125 \mathrm{~km}$. The VIRTIS abundance of CO is also systematically larger than the model prediction, especially around the subsolar point.

Unfortunately the VIRTIS observations at $4.3 \mu \mathrm{m}$ have not yet been exploited to derive atmospheric densities and variations of $\mathrm{CO}_{2}$. In contrast to the $\mathrm{CO}$ emissions, dozens of $\mathrm{CO}_{2}$ bands contribute to the observed emission, many of them very optically thick up to well into the thermosphere, all of which makes the forward model calculations and the interpretation more difficult. Still, such retrievals will be very valuable, not only to derive $\mathrm{CO}_{2}$ densities but also if they are combined with the $\mathrm{CO}$ retrievals. They also offer the possibility to determine atmospheric temperatures (López-Valverde et al. 2011a). One further advantage is the strong emission, compared to the $\mathrm{CO}$ fluorescence, which will permit studying the Venus thermosphere up to $160 \mathrm{~km}$ at least.

Krasnopolsky (2014a) proposed deriving CO densities at a given mesospheric altitude routinely from ground observations of these airglow emissions, and he did so for the $\mathrm{CO}$ abundance at about $104 \mathrm{~km}$ from the $\mathrm{CO}(2-1)$ first hot band. He found a constant $\mathrm{CO}$ mixing ratio from $50^{\circ} \mathrm{S}$ to $50^{\circ} \mathrm{N}$ with a mean value of $560 \pm 100 \mathrm{ppm}$. However, this technique is prone to large errors if the variations of the altitude/pressure relation of the emitting layer is not taken into account. Also the large variation in $\mathrm{CO}$ volume mixing ratio (vmr) over the width of typical weighting functions (several scale heights) presents a second difficulty in nadir (ground based) sounding for $\mathrm{CO}$. This was indeed one of the major error sources considered by Krasnopolsky (2014a). An additional caution is that this airglow will permit precise determinations of VMR (relative abundances) only if other emission bands (from $\mathrm{CO}_{2}$ ) are available simultaneously; otherwise the vmr results will be model dependent. For all these reasons, whenever possible limb sounding from orbit such as that from VIRTIS (Gilli et al. 2015) should be the ideal strategy for sounding of CO in the Venus upper atmosphere.

\subsection{Atomic Oxygen}

The distribution of oxygen atoms in the Venus nightside upper mesosphere was only poorly known following the Pioneer Venus and Soviet missions to Venus. The PV neutral mass spectrometer only provided composition measurements down to $\sim 145 \mathrm{~km}$. The VTS 3 model (Hedin et al. 1983) extrapolated downward the O density measured in the thermosphere assuming hydrostatic equilibrium strongly and therefore overestimated the $\mathrm{O}$ abundance below $\sim 95 \mathrm{~km}$ where $\mathrm{O}$ atoms chemically recombine. Only models coupling photochemistry and vertical transport (Massie et al. 1983; Bougher and Borucki 1994) were able to predict the presence of a density maximum in the mesosphere-thermosphere transition region. Extensive limb and nadir observations with VIRTIS have made it possible to use the observed distribution of the $\mathrm{O}_{2}\left(\mathrm{a}^{1} \Delta_{\mathrm{g}}\right)$ airglow to determine the vertical and horizontal statistical distribution of atomic oxygen on the Venus nightside. The 3-body recombination process:

$$
\mathrm{O}+\mathrm{O}+\mathrm{M} \rightarrow \mathrm{O}_{2}\left(\mathrm{a}^{1} \Delta_{\mathrm{g}}\right)+\mathrm{M}
$$

has been identified as the dominant source of excited oxygen molecules in the night mesosphere. Although the fraction of molecules directly formed in the $\mathrm{a}^{1} \Delta_{\mathrm{g}}$ metastable state is small, radiative cascades from higher-lying states and possibly collisional relaxation produces a high effective branching ratio $\varepsilon$. The role of quenching of the $\mathrm{a}^{1} \Delta_{\mathrm{g}}$ state by $\mathrm{CO}_{2}$ is limited to the bottom of the emitting layer and only an upper limit of the quenching coefficient by $\mathrm{CO}_{2}$ has been determined. Once the rate coefficient $\mathrm{k}$ and its temperature 
Fig. 12 Distribution of the $\mathrm{O}$ density at $103 \mathrm{~km}$ derived from global airglow observations at $1.27 \mu \mathrm{m}$ with the VIRTIS spectral imager. The $\mathrm{CO}_{2}$ density used to convert airglow intensity into oxygen density was taken from a compilation of $\mathrm{CO}_{2}$ profiles derived from stellar occultation measurements with the SPICAV instrument (from Soret et al. 2012a)

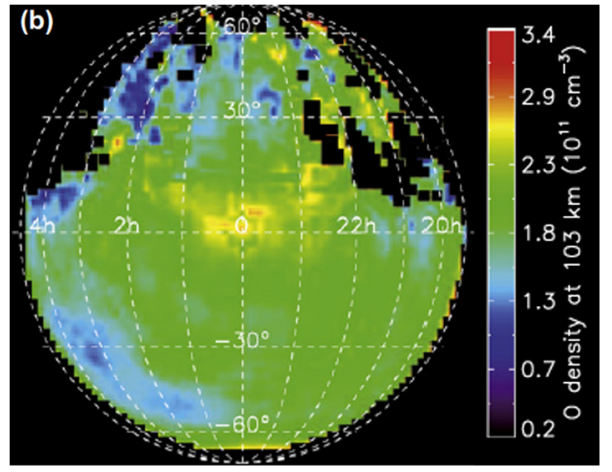

dependence and third body identity and density are known, observations of the emission profile provide a direct measurement of the $\mathrm{O}$ density distribution. The steady state $\mathrm{O}$ density profile is then obtained from the equality between the $\mathrm{O}_{2}\left(\mathrm{a}^{1} \Delta_{\mathrm{g}}\right)$ chemical production and loss rate by radiative relaxation and collisional quenching with $\mathrm{CO}_{2}$ :

$$
[\mathrm{O}](z)=\sqrt{\left[\mathrm{O}_{2}^{*}\right](z) \frac{\mathrm{A}+\mathrm{C}_{q}\left[\mathrm{CO}_{2}\right](z)}{k \varepsilon\left[\mathrm{CO}_{2}\right](z)}}
$$

This technique was developed by Gérard et al. (2009a) who used $\varepsilon=0.75$, k was taken equal to $3.1 \times 10^{-32} \mathrm{~cm}^{6} \mathrm{~s}^{-1}$ and the quenching coefficient $\mathrm{Cq}=1 \times 10^{-20} \mathrm{~cm}^{3} \mathrm{~s}^{-1}$. It was applied by Soret et al. (2012a) who used the full VIRTIS database to build up maps of the statistical three-dimensional $\mathrm{O}$ density between $\sim 90$ and $120 \mathrm{~km}$. They found that the $\mathrm{O}_{2}\left(\mathrm{a}^{1} \Delta_{\mathrm{g}}\right)$ hemispherically averaged density at $99.2 \mathrm{~km}$ is $2.1 \times 10^{9} \mathrm{~cm}^{-3}$, with a maximum value of $6.5 \times 10^{9} \mathrm{~cm}^{-3}$. The dominant third body in the recombination process is the $\mathrm{CO}_{2}$ molecule, whose density profiles were taken from the empirical VTS3 model or from a sample of SPICAV stellar occultations. The $\mathrm{O}$ hemispheric average peak density was $1.9 \times$ $10^{11} \mathrm{~cm}^{-3}$ in both cases. The maximum density is located at $\sim 106 \mathrm{~km}$ with the $\mathrm{CO}_{2}$ from the VTS3 model and $\sim 103 \mathrm{~km}$, with the values derived from SPICAM. Figure 12 shows that the highest densities of atomic oxygen are found around the antisolar point, in agreement with the $\mathrm{O}_{2}\left(\mathrm{a}^{1} \Delta_{\mathrm{g}}\right)$ airglow distribution. It was also shown that the results are relatively insensitive to the values of both $\mathrm{Cq}$ and $\varepsilon$ when they remain within the range of previously estimated values.

These results are in fair agreement with the 3-D modeled peak values of $2.8 \times 10^{11} \mathrm{~cm}^{-3}$ at $104 \mathrm{~km}$ by Brecht et al. (2012) and $2.0 \times 10^{11} \mathrm{~cm}^{-3}$ at $110 \mathrm{~km}$ in the 1 -D model by Krasnopolsky (2010) respectively (Fig. 13). Comparing the $\mathrm{O}$ density map derived by Soret et al. (2012a) from the oxygen nightglow observations with those predicted by the VTS3 model, it appears that the altitude dependence is very different and that the densities based on the $\mathrm{O}_{2}$ airglow are substantially higher above $95 \mathrm{~km}$. A detailed comparison with values calculated with the 3-D model will be made in Sect. 5 .

No further dayside observations related to the atomic oxygen distribution was obtained from the Venus Express mission. Global dayside images from PVOUVS (Alexander et al. 1993) of the oxygen emission at $130.4 \mathrm{~nm}$ has lead to the conclusion that the O density is asymmetric in local time, with higher values toward the evening terminator. This asymmetry was interpreted as a consequence of enhanced eddy mixing in the morning hours leading to lower thermospheric $\mathrm{O}$ densities in this sector. 
Fig. 13 Comparison of the $\mathrm{O}$ density derived from the VIRTIS oxygen nightglow measurements at $24^{\circ} \mathrm{S}, 00: 44 \mathrm{LT}$ with the profiles from the VTS3 model (dotted line) and from the global 1-D model of Krasnopolsky (2010) (dashed line). The two profiles derived from the $\mathrm{O}_{2}{ }^{1} \Delta_{\mathrm{g}}$ airglow observations use the VTS3 $\mathrm{CO}_{2}$ density (long dashes) and the SPICAV compilation (solid line) respectively (from Soret et al. 2012a)

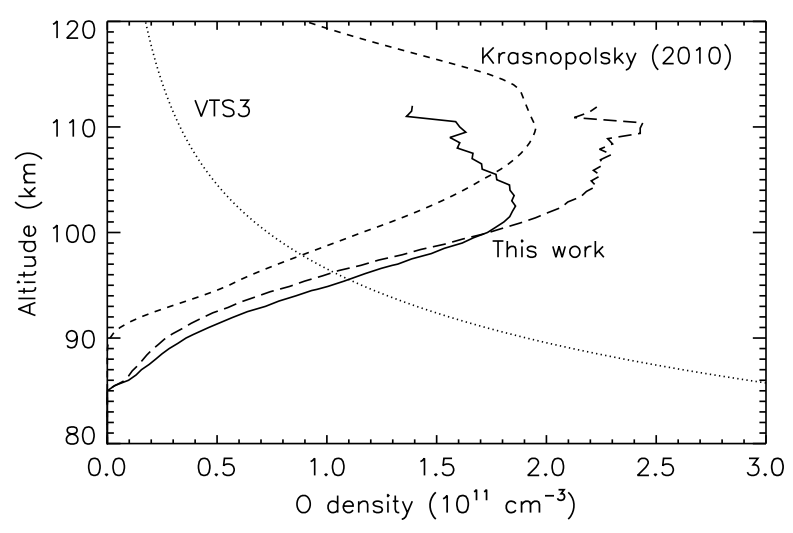

\subsection{Atomic Nitrogen}

The density of $\mathrm{N}\left({ }^{4} \mathrm{~S}\right)$ ground-state atoms was measured with the Pioneer Venus neutral mass spectrometer above $145 \mathrm{~km}$ where its vertical distribution is controlled by diffusive equilibrium. However, as for atomic oxygen, models predict that the $\mathrm{N}$ density peaks at a lower altitude, inaccessible so far to in situ measurements. Therefore the nightside $\mathrm{N}$ density profile in the lower thermosphere can only be indirectly determined through modeling of the nitric oxide airglow distribution produced by radiative association $\mathrm{N}\left({ }^{4} \mathrm{~S}\right)+\mathrm{O}\left({ }^{3} \mathrm{P}\right) \rightarrow \mathrm{NO}^{*}$. The volume emission rate of the $\delta$ and $\gamma$ bands is directly proportional to the $[\mathrm{O}] \times[\mathrm{N}]$ density product and is directly obtained from $\eta(\mathrm{NO})=\mathrm{k}_{\mathrm{NO}}[\mathrm{O}][\mathrm{N}]$, where $\eta(\mathrm{NO})$ is the volume emission rate of $\mathrm{NO} \delta$ and $\gamma$ bands and $\mathrm{k}_{\mathrm{NO}}$ is the two-body recombination coefficient. $\mathrm{N}$ vertical distributions reproducing NO nightglow limb observations were calculated with a one-dimensional chemical-transport model by Gérard et al. (2008b). They show a maximum density $\sim 2-5 \times 10^{8} \mathrm{~cm}^{-3}$ between 115 and $120 \mathrm{~km}$. Stiepen et al. (2012) modeled the average nightside conditions and calculated a peak of $8 \times 10^{8} \mathrm{~cm}^{-3}$ at $122 \mathrm{~km}$. The nitrogen density distribution was also calculated with the VTGCM (Brecht et al. 2011) at all local times and latitudes.

\subsection{Thermospheric Ozone}

Ozone is expected to play a key role in the excitation of the $\mathrm{OH}$ Meinel bands through the Bates-Nicolet mechanism:

$$
\mathrm{O}_{3}+\mathrm{H} \rightarrow \mathrm{OH}^{*}(v<10)+\mathrm{O}_{2}
$$

where the $\mathrm{OH}$ molecule is produced in vibrationally excited level up to $v=9$. Another process involving the reaction between $\mathrm{HO}_{2}$ and $\mathrm{O}$ was found to be less important in the Venus (Krasnopolsky 2010) as in the Earth's upper atmosphere. Following the discovery of the presence of the $\mathrm{OH}$ Meinel bands in the Venus nightglow by Piccioni et al. (2009), Krasnopolsky (2010) detected the OH (1-0) P1(4.5) and (2-1) Q1(1.5) airglow lines from the ground. It was suspected that ozone was indeed present near $95 \mathrm{~km}$ in sufficient amount to produce the intensity observed at the limb with VIRTIS. Montmessin et al. (2011) sporadically detected near $100 \mathrm{~km}$ a broad absorption band near $255 \mathrm{~nm}$ during stellar absorption measurements with the SPICAV-UV spectrograph. They derived mean peak values $\sim 7 \times 10^{7} \mathrm{~cm}^{-3}$ at $95 \mathrm{~km}$ and a layer thickness of $\sim 7 \mathrm{~km}$ at half maximum. In a detailed 
analysis of the brightness of the $\mathrm{OH}$ infrared bands, Soret et al. (2012b) averaged a few thousands limb profiles and obtained a mean peak intensity of $350_{-210}^{+350} \mathrm{kR}$ for the $\Delta v=1$ sequence. They showed that these ozone densities could only be reconciled with the $\mathrm{OH}$ airglow observations if the measured $\mathrm{O}_{3}$ densities were upper limits while lower densities were not detectable with SPICAV. Both studies concluded that the model atmosphere by Krasnopolsky (2010) predicted an excessive amount of ozone reaching $4 \times 10^{9} \mathrm{~cm}^{-3}$ at $94 \mathrm{~km}$. Based on these elements, Krasnopolsky (2013) revised his 2010 model and increased the load in chlorine compounds. He added 25 chemical reactions, increased the flux of $\mathrm{H}$ atoms at the upper boundary and imposed a downward flux of chlorine atoms of $1 \times 10^{10} \mathrm{~cm}^{-2} \mathrm{~s}^{-1}$ through the upper boundary. The calculated $\mathrm{O}_{3}$ density decreased, reaching a peak of about $1 \times 10^{8} \mathrm{~cm}^{-3}$ at $93 \mathrm{~km}$. The case of the Venus $\mathrm{OH}$ infrared airglow clearly illustrates how airglow observations can improve our understanding of aeronomical processes in the Venus upper atmosphere.

Following these modifications, the ozone density decreased and the model agreed with the observational constraints for the mean nighttime atmosphere.

\subsection{Atomic Carbon and $\mathrm{O}_{2}$}

The carbon density has never been directly measured by mass spectrometer in the Venus thermosphere. Estimates entirely rely on dayglow measurements and neutral and ionospheric models. Carbon atoms are mainly lost through the $\mathrm{C}+\mathrm{O}_{2} \rightarrow \mathrm{CO}+\mathrm{O}$ reaction. The $\mathrm{C}$ density is closely controlled by the $\mathrm{O}_{2}$ abundance and therefore, its value has been used to estimate the mixing ratio of molecular oxygen. Estimation of the carbon density combined with a detailed photochemical modeling has been the only method available up to now to evaluate the $\mathrm{O}_{2}$ density in the lower thermosphere. From limb scan observations of the dayglow of the $156.1 \mathrm{~nm}$ and $165.7 \mathrm{~nm}$ multiplets with PV-OUVS, Paxton (1985) estimated the $\left[\mathrm{O}_{2}\right] /\left[\mathrm{CO}_{2}\right]$ to be $\sim 3 \times 10^{-3}$. Fox and Paxton (2005) determined the $\mathrm{O}_{2}$ mixing ratio best fitting those airglow observations and the $\mathrm{C}^{+}$ion density to be slightly larger than $3 \times 10^{-4}$. Measurements of the distribution of the intensity of the carbon dayglow emissions at 126.1, 156.1, and $165.7 \mathrm{~nm}$ with UVIS on board Cassini by Hubert et al. (2012) raised again the question of the $\mathrm{O}_{2}$ abundance in the Venus lower thermosphere. They concluded that the carbon density profile by Fox and Paxton (2005) fell short by a factor of $\sim 2$ to $\sim 10$ to match the observed CI UV line intensity. They concluded that the $\left[\mathrm{O}_{2}\right] /\left[\mathrm{CO}_{2}\right]$ mixing ratio was probably less than $3 \times 10^{-4}$. For comparison, in the model of Krasnopolsky and Parshev (1983) predicted a $\left[\mathrm{O}_{2}\right] /\left[\mathrm{CO}_{2}\right]$ ratio $\sim 6 \times 10^{-4}$ near $140 \mathrm{~km}$. The nightside model by Krasnopolsky (2013) predicts a ratio $\sim 8 \times 10^{-4}$ at $130 \mathrm{~km}$.

\subsection{Temperature Retrievals from Non-LTE Fluorescence Emissions}

The intensity of non-LTE emissions, by definition, does not contain much information about the local temperature. However there are other effects allowing the sounding of the temperature from these emissions, if spectral resolution and sensitivity permit it. As a first example it is possible to learn about the kinetic temperature from the rotational distribution of a given ro-vibrational band, if the spectral resolution is high enough to permit such an observation and if rotational LTE is assumed. This is the case of the CO emissions at $4.7 \mu \mathrm{m}$ by VIRTIS/Venus Express. Other examples are the dependence of collisional quenching rates on temperature, and of the absorption of solar radiation on the width of the spectral lines. One example of emission where all these effects do have an impact is the $\mathrm{CO}_{2}$ strong band system at $4.3 \mu \mathrm{m}$. Both cases are examined next. 


\subsubsection{CO Rotational Distribution at 4.7 Microns}

The non-LTE emissions of CO at $4.7 \mu \mathrm{m}$ observed by VIRTIS contain information about the whole structure of the two CO bands involved, the fundamental and the first-hot band, and this structure is dependent on the rotational temperature. As far as the rotational distribution is maintained in LTE, which is normally the case up to the thermosphere, this opens the possibility to retrieve also the atmospheric temperature from this data. The data-model fit is complex because both bands contribute at the same time and many of the lines are a mixture of two emissions with different populations. However the non-LTE forward model is able to incorporate this, and it was finally possible to retrieve $\mathrm{CO}$ abundance and temperatures simultaneously (Gilli 2012; Gilli et al. 2015), although with typical error bars of 30 K. Figure 14 shows an example of the temperatures obtained with this method.

The results are even noisier than the retrievals of the $\mathrm{CO}$ density shown before, but these results concern layers difficult to sound during daytime otherwise, and therefore supply a valuable dataset to study the thermal and dynamical structure of the Venus thermosphere. The selected results presented here show an agreement between VIRTIS and the VTGCM at most altitudes, within large error bars, but with a poorer match at equatorial latitudes below 120-130 km. Here, significantly colder upper mesosphere temperatures persist in the VIRTIS data. This is a surprising result, especially where the model predicts a local maximum in temperature. Also simpler 1-D models of radiative equilibrium predict a warm or mesopeak layer at these altitudes. One important aspect is that both $\mathrm{CO}$ and temperature come from the same VIRTIS dataset and should be explained simultaneously, which currently represents a challenge. The CO global results, at least below $130 \mathrm{~km}$, are consistent with a CO distribution controlled by dynamics, with a strong sub-solar to anti-solar gradient. Similar variations are found with the VTGCM although the VIRTIS CO values are systematically larger. However, the VIRTIS temperature structure in the subsolar point does not resemble the VTGCM. Gilli et al. (2015) explored various explanations but none seems to be satisfactory. Whether the two data-model discrepancies (CO diurnal variations and equatorial temperatures) are somehow related or they point to deficiencies in the data and/or in the models has not been determined. Gilli et al. also pointed out that other VIRTIS datasets remain unexploited, which could give new temperature results in this altitude range. The first
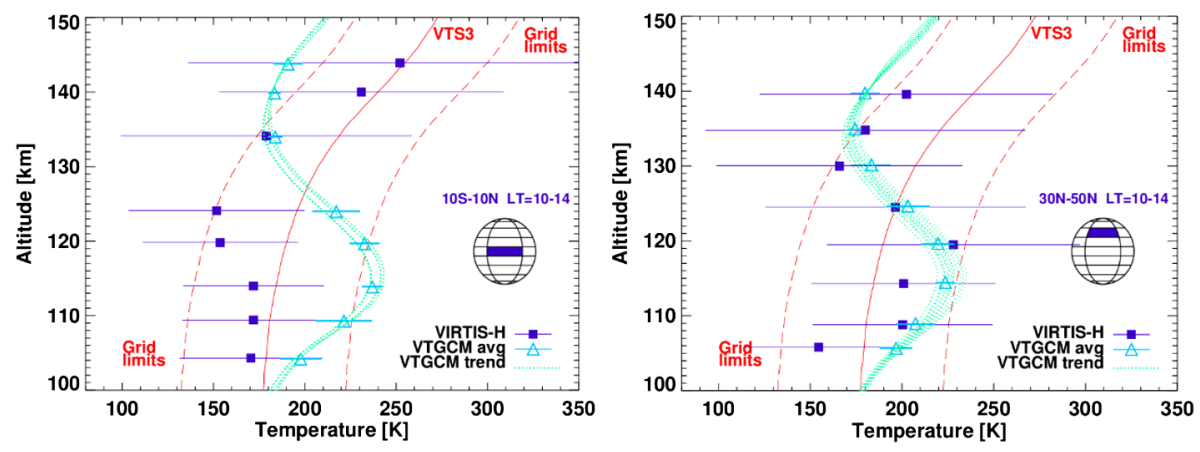

Fig. 14 Retrieval of the Venus kinetic temperature at noon (10:00-14:00 LT) in two latitude bands, as indicated, from averaged spectra of the VIRTIS-H $4.7 \mu \mathrm{m}$ non-LTE limb measurements. Light-blue triangles and lines: VTGCM values averaged for the same latitude and local time boxes. Individual profiles from the GCM within the box are also shown (labeled "trend"). The VTS3 reference profile is shown for comparison (from Gilli et al. 2015) 
of them are the limb $\mathrm{CO}_{2}$ emissions at $4.3 \mu \mathrm{m}$ mentioned above. The second focuses upon nadir emissions at $4.3 \mu \mathrm{m}$.

Regarding ground-based measurements, and as mentioned above, the $\mathrm{CO}(2-1)$ band airglow was recently used by Krasnopolsky (2014a) to explore the thermal structure in the Venus upper atmosphere. Krasnopolsky (2014a) derived rotational temperatures from only four adjacent rotational transitions. The procedure is sound but contains many uncertainties and assumptions, as discussed before in Sect. 2.7.3. He found a very small or negligible latitudinal variation around $110 \mathrm{~km}$ (claiming slightly higher values on the Southern Hemisphere are well within error bars) and a mean value of $203 \pm 9 \mathrm{~K}$. This is significantly warmer than the $189 \pm 8 \mathrm{~K}$ obtained at the $100-110 \mathrm{~km}$ range from the whole $2-1$ band by Crovisier et al. (2006), which were already $10 \mathrm{~K}$ warmer than VIRA at that altitude. This means a $20 \mathrm{~K}$ difference with respect to VIRA. Still, the known dispersion in previous determinations and in model predictions (Krasnopolsky 2014a; Gilli et al. 2015) is larger than those differences, which means that our understanding of the thermal structure and its variability at these altitudes is not complete yet.

\subsubsection{Nadir Mapping of $\mathrm{CO}_{2}$ Solar Fluorescence at $4.3 \mu \mathrm{m}$}

The $\mathrm{CO}_{2}$ system of ro-vibrational bands at $4.3 \mu \mathrm{m}$ is so optically thick in the Venus atmosphere that in a nadir geometry the atmospheric emission originates from very high in the atmosphere, around the mesopause and above, where the solar fluorescence is already very strong. This situation is well known and common to both $\mathrm{CO}_{2}$ atmospheric planets, Venus (López-Valverde et al. 2007) and Mars (López-Valverde et al. 2005). While the VIRTIS limb observations have not been exploited for this purpose, the nadir emission has recently been studied to derive temperatures in that atmospheric region by Peralta et al. (2016).

Figure 15 shows a map of VENUS thermospheric temperatures after gathering a set of observations of VIRTIS-H spanning the whole Venus Express mission. The actual atmospheric region examined extends from 100 to $150 \mathrm{~km}$, which is very broad and this needs to be taken into account when comparing models and data. Peralta et al. (2016) actually performed such a comparison with a careful extraction of results of the Venus Thermospheric
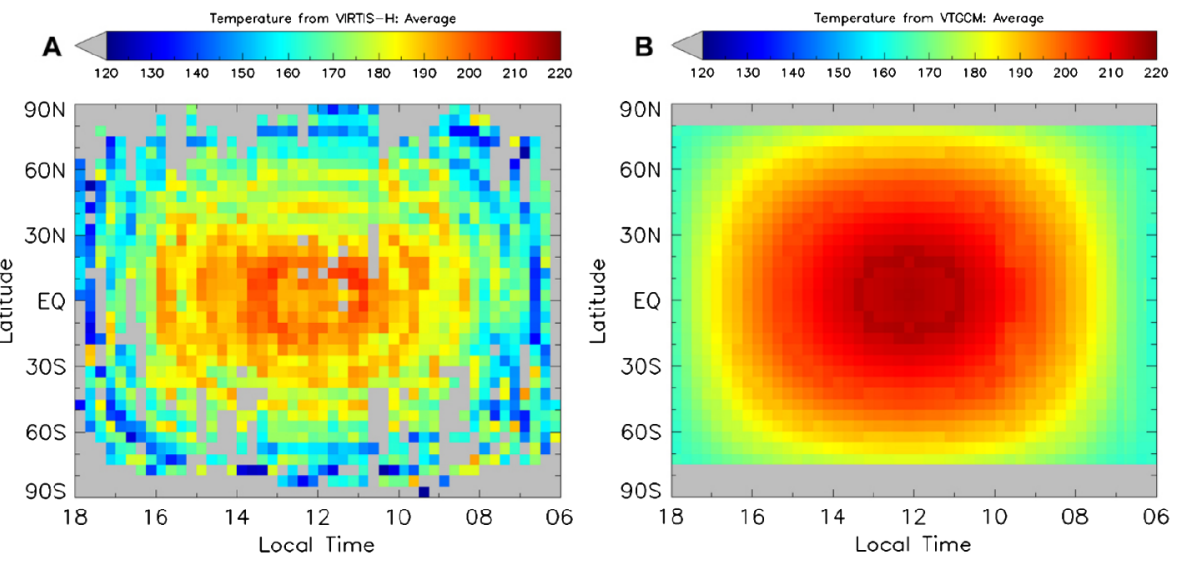

Fig. 15 Left panel: Atmospheric temperature in the upper mesosphere and lower thermosphere of Venus $\left(0.01-10^{-5} \mathrm{mb}\right)$ during daytime, as obtained from the $\mathrm{CO}_{2}$ NLTE nadir spectra taken by VEx/VIRTIS-H. Right panel: dayside temperatures from the VTGCM by Brecht and Bougher (2012) and averaged for the same pressure interval. After Peralta et al. (2016) 
GCM (Brecht and Bougher 2012). The global view is a very good agreement in the latitudinal and local time variations, although the observed temperatures seem systematically colder than the model.

\section{Dynamics and Transport in the Upper Atmosphere}

A "first-order" picture of the Venus upper atmosphere circulation and the resulting transport of species have been gleaned from numerous remote and in situ datasets collected mostly at the planet. Recent progress in our understanding of the Venus dynamics have been reviewed by Sánchez-Lavega et al. (2017). However, the underlying physical processes maintaining this mean circulation, and driving its large variations, are poorly understood. In fact, this large variability of the upper atmosphere global winds is one of the outstanding questions regarding Venus' circulation (Bougher et al. 2006; Schubert et al. 2007). Direct wind measurements are provided by ground-based observations; Venus spacecraft measurements yield proxies that are typically used to derive the magnitude and variability of these mesospherethermosphere winds.

\subsection{Observations of Night Airglow Emissions in the Mesosphere-Thermosphere Transition Region}

Extensive measurements of the $\mathrm{O}_{2}\left(\mathrm{a}^{1} \Delta_{\mathrm{g}}\right)$ and NO nightglows at the nadir were assembled to produce global statistical maps of the horizontal distribution of the two emissions. Figure 16 summarizes the result of these syntheses. The $\mathrm{O}_{2}$ airglow distribution is characterized by a bright area of emission centered near the antisolar point. (Gérard et al. 2008c; Piccioni et al. 2009; Soret et al. 2012a). The contrast between the intensity in this region and the weaker airglow is about a factor of 5. Following correction for the thermal background emission, the hemispheric area-weighted intensity is $\sim 500 \mathrm{kR}$. Shakun et al. (2010) obtained a mean intensity of $1.0 \pm 0.4 \mathrm{MR}$ after applying corrections for the Venus topography and radiative transfer. They found a secondary bright region at latitudes between $30^{\circ}$ and $60^{\circ}$ in the southern hemisphere near 23:00 LT. By contrast, the NO night airglow maximum intensity is shifted by about 3 hours toward the dawn meridian, with a hemispheric average total intensity of $1.9 \mathrm{kR}$. The two emissions originate from different altitudes. The NO band volume emission rate peaks at $115 \pm 7 \mathrm{~km}$ (Gérard et al. 2008b; Stiepen et al. 2013), in close agreement with previous limb observations near periapsis with PV-OUVS (Gérard et al. 1981). The $\mathrm{O}_{2}$ emission at $1.27 \mu \mathrm{m}$ shows a maximum volume emission rate at 99.2 $\pm 1.0 \mathrm{~km}$ for $\mathrm{O}_{2}\left(\mathrm{a}^{1} \Delta_{\mathrm{g}}\right.$ ) (Soret et al. 2012a), thus statistically located $16 \mathrm{~km}$ below the NO airglow peak.

Differences of global morphology between the two emissions have been interpreted as resulting from a different global circulation pattern between the altitudes of the two emissions. The symmetry around the subsolar point suggests that, in the $99 \mathrm{~km}$ region, the zonal wind is statistically quite weak, while its picks up speed again at higher altitudes where it displaces the NO emission towards the morning sector. The reason for this unexpected increase of the zonal wind velocity is still largely debated. One possibility is that $\mathrm{O}^{+}$ions moving westward (in the superrotation direction) on the nightside (Lundin et al. 2013) exchange momentum with the ambient neutral gas down to the lower thermosphere and cause the observed displacement of the NO airglow.

In any case, individual snapshot images of the $\mathrm{O}_{2}\left(\mathrm{a}^{1} \Delta_{\mathrm{g}}\right)$ airglow distribution in the nadir and limb directions have shown that the airglow is patchy with bright regions observed at 


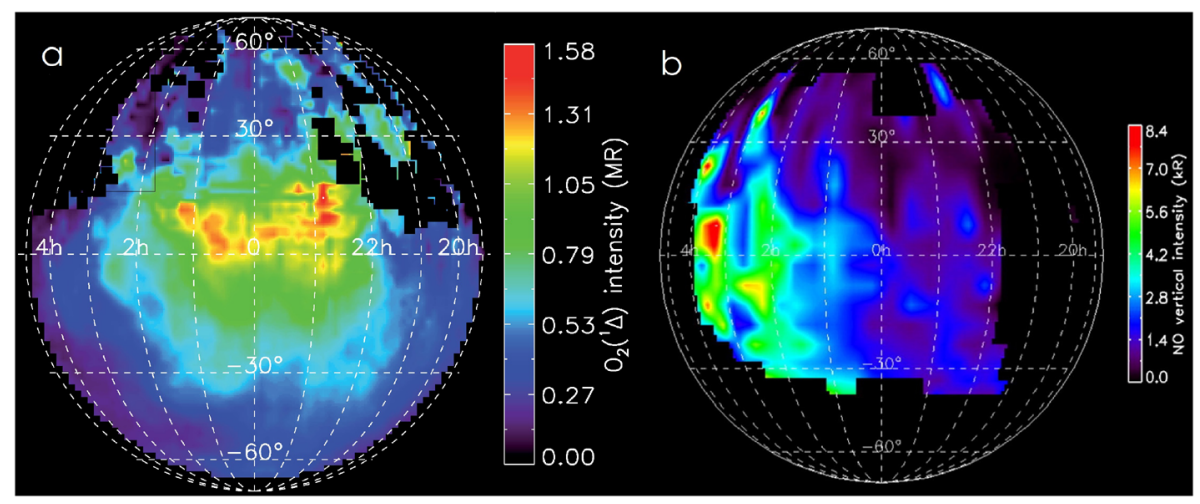

Fig. 16 Maps of the statistical distribution of the $\mathrm{O}_{2}$ and NO nightglow intensity. The center of the circle corresponds to the antisolar point. The difference between the bright spot location of $\mathrm{O}_{2}$ and NO emission suggests two different dominant wind regimes at 99 and $115 \mathrm{~km}$, with zonal wind speeds dropping to low values in the upper mesosphere, picking up speed again at higher altitude (see Brecht et al. 2011) (from Soret et al. 2012a and Stiepen et al. 2013)

different local times and latitudes (Hueso et al. 2008; Piccioni et al. 2009; Gérard et al. 2009a; Soret et al. 2014). This result is reminiscent of the scattered distribution of bright patches in the NO FUV nightglow (Bougher et al. 2006). The quasi-symmetry shown in Fig. 16a only appears when a large enough number of individual images are summed up (Soret et al. 2014).

Simultaneous nadir observations of the latitudinal intensity distribution of the $\mathrm{O}_{2}\left(\mathrm{a}^{1} \Delta_{\mathrm{g}}\right)$ with VIRTIS and NO airglow with SPICAV by Gerard et al. (2009b) have shown that the two emissions are not spatially correlated. This result confirms that the dynamical regimes controlling the $\mathrm{O}$ density at $99 \mathrm{~km}$ and the $\mathrm{N}$ density at $115 \mathrm{~km}$ may be quite different. The time required for $\mathrm{O}$ atoms to move down between the two emission levels depends on the strength of the vertical velocity component but is probably on the order of an Earth day. When combined with a typical horizontal velocity of $50 \mathrm{~m} \mathrm{~s}^{-1}$, the atoms are expected to travel horizontally over long distances. These characteristics probably explain the lack of correlation between bright spots originating from the two atmospheric levels, especially in the presence of a wind shear. Numerical simulations with a two-dimensional model by Collet et al. (2010) demonstrated the large horizontal distance between enhanced regions of $\mathrm{O}$ and $\mathrm{N}$ densities at the two levels following an injection of a blob of $\mathrm{O}$ and $\mathrm{N}$ atoms in the presence of a horizontal wind of several tens of $\mathrm{m} \mathrm{s}^{-1}$.

\subsection{Airglow Morphology and Variability}

\subsubsection{Dayside}

As discussed in Sect. 2.3, Masunaga et al. (2015) obtained three time sequences of brightness variations of the Venus dayglow with the EXCEED telescope. They found a 4.5-day modulation in all three emissions and secondary periodicities during the first time interval. Periodicities of 1.1-day in the second interval, and 1.0 and 11-day during the third interval were also identified. The amplitudes at $135.6 \mathrm{~nm}$ were $6.6-10 \%$ and suggested that these modulations are caused by density oscillations of oxygen atoms or photoelectrons in the thermosphere. They argued that planetary-scale waves and/or gravity waves propagating 
from the middle atmosphere, and/or minor periodic variations of the solar EUV radiation flux can play a role. They also investigated possible effects of the solar wind parameters on the dayglow variations using the ASPERA-4 and magnetometer instruments on board Venus Express. They found no clear correlation with the dayglow variations but claimed that their minor periodicities are similar to the dayglow periodicities. We note that the periods they identified are shorter than the $30-50 \%$ thermospheric density periodicities detected by Forbes and Konopliv (2007) from radar tracking of the Magellan spacecraft.

\subsubsection{Nightside}

Statistical views of the global NO and $\mathrm{O}_{2}\left(\mathrm{a}^{1} \Delta_{\mathrm{g}}\right)$ airglow morphology are useful as they provide datasets which are directly comparable to steady state simulations made with general circulation models. However, they considerably differ from the instantaneous morphology that would be obtained if global snapshots can be obtained. The statistical distribution obtained after averaging a large number of individual observations is somewhat misleading as it masks out the strong variability of these airglow structures. By contrast, snapshot nadir or limb observations of both the $\mathrm{O}_{2}$ IR and the NO UV emissions indicate that the distribution described in Fig. 9 is actually rarely observed. Therefore, averaged global maps do not reflect the short-term variability that is an important component of the dynamics of the Venus upper atmosphere. This aspect was analyzed using the $\mathrm{O}_{2}\left(\mathrm{a}^{1} \Delta_{\mathrm{g}}\right)$ limb and nadir observations and, to a lesser extent, the NO observations with SPICAV.

Soret et al. (2014) analyzed the evolution of regions of enhanced $1.27 \mu \mathrm{m}$ airglow extracted from nadir VIRTIS images. They showed that the regions of bright $\mathrm{O}_{2}\left(\mathrm{a}^{1} \Delta_{\mathrm{g}}\right)$ airglow may be located almost anywhere on the nightside and move in latitude and local time over period of a few hours, even though the brightest spots tend to occur near the antisolar point. An organized structure similar to Fig. 16 only appears following addition of a large number of VIRTIS nadir spectral images collected over periods of a few months. They followed the intensity and the displacement of bright patches over time to determine apparent wind velocities and characteristic decay or rise times. They derived speeds of the displacements varying from 0 to $213 \mathrm{~m} \mathrm{~s}^{-1}$, with a mean value of $54 \mathrm{~m} \mathrm{~s}^{-1}$ (Fig. 17). The high variability of both the direction and the absolute value of the horizontal velocity vector associated with the displacements made it impossible to extract a picture of the global circulation in the 95$100 \mathrm{~km}$ regime. The mean measured decay time of $\sim 750 \mathrm{~min}$ is considerably longer than the 75 -min lifetime of the metastable $\mathrm{a}^{1} \Delta_{\mathrm{g}}$ state. It is not too different from the $\sim 35$ hours

Fig. 17 Apparent horizontal wind vectors on the Venus nightside (dashed area) derived from the displacement of bright patches of $1.27 \mu \mathrm{m}$ airglow. The plot shows a very large variability in space and time (from Soret et al. 2014)

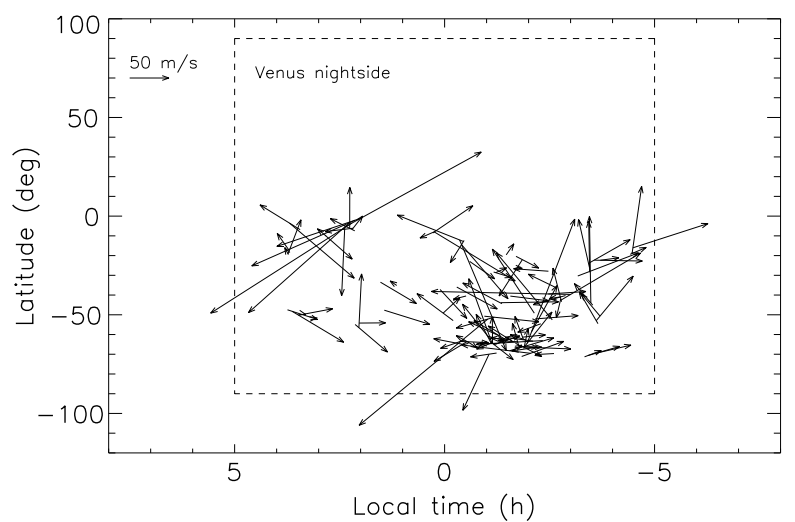


obtained in the two-dimensional simulation by Collet et al. (2010). Soret et al. (2014) explained it as a combination of the excited $\mathrm{O}_{2}$ radiative decay time and the chemical lifetime of the $\mathrm{O}$ atoms supplied by the global circulation and its vertical transport efficiency. The vertical-latitudinal brightness distribution is equally variable as was shown by Gérard et al. (2009a, 2014). Bright spots of $\mathrm{O}_{2}\left(\mathrm{a}^{1} \Delta_{\mathrm{g}}\right)$ emission correspond to regions of enhanced supply of oxygen atoms. However, at this point, it is difficult to specify whether these are signatures of perturbations of the SSAS general circulation caused by propagating gravity waves or if increased local vertical transport strength acts to move these atoms downward.

Another potential source of variability is the solar EUV flux that dissociates $\mathrm{CO}_{2}$ on the dayside and is therefore expected to control the total amount of $\mathrm{O}$ atoms carried to the nightside. The EUV solar flux between 0.1 and $50 \mathrm{~nm}$ measured by the SEM index only varied by $14 \%$ during the period of VIRTIS observations. However, Soret and Gérard (2015) did not observe any correlation between the $\mathrm{O}_{2}$ nightglow intensity and the solar EUV flux during solar minimum conditions. They concluded that internal factors such as wave activity mask out the solar control of the oxygen night airglow variability. The intensity and the latitudinal distribution of the nitric oxide night airglow is also highly time variable within a limited local time sector. Stiepen et al. (2013) illustrated sequences of NO measurements along the SPICAV instrument line of sight on the atmosphere They showed examples of NO airglow nadir brightness measured along three latitudinal cuts, all made between 02:00 and 03:00 LT in the sector of the statistical bright spot. The three intensity curves clearly exhibit drastically different intensities and latitudinal variations. These observations are in agreement with the day-to-day variability of the NO airglow in images taken 24 hours apart with PV-OUVS by Stewart et al. (1980).

\subsection{Wind Measurements (and Wind Proxies) in the Thermosphere and Mesosphere}

From past observations it has been noted that Venus has a unique two-part circulation pattern within the upper atmosphere (See Fig. 18) (e.g., Bougher et al. 1997, 2006; Lellouch et al. 1997; Schubert et al. 2007). One part occurs in the region between the surface and the top of the cloud deck $(\sim 70 \mathrm{~km})$. This region is dominated by a stable wind pattern flowing in the direction of the planet's spin and is faster than Venus' rotation. This flow is known as a retrograde superrotating zonal flow (RSZ). The second part occurs above $\sim 120 \mathrm{~km}$ and is a relatively stable mean subsolar-to-antisolar flow (SS-AS). Venus has inhomogeneous heating in the upper atmosphere by solar radiation (EUV, UV, IR) thus providing huge pressure gradients to generate this dominant SS-AS flow pattern (e.g. Schubert et al. 2007; Bougher et al. 1997, 2006). In the altitude range of $\sim 70-120 \mathrm{~km}$ (i.e. the transition region), the two major flow patterns are superimposed, often with large variations on short timescales.

This "first-order" picture of Venus' upper atmosphere winds has been gleaned from a number of remote and in-situ datasets collected at the planet. A thorough examination of Pioneer Venus Orbiter (PVO) neutral density (e.g., $\mathrm{CO}_{2}, \mathrm{O}, \mathrm{He}$, and $\mathrm{H}$ ) and temperature distributions above $\sim 130 \mathrm{~km}$, as well as ultraviolet (UV) NO nightglow and $\mathrm{O}$ dayglow distributions, has been used to constrain general circulation model (GCM) simulations, from which SS-AS and RSZ wind magnitudes can be extracted (see reviews by Bougher et al. 1997, 2006). In addition, visible and infrared $\mathrm{O}_{2}$ nightglow distributions from Venera 9 and 10, Galileo, PVO and the ground, along with minor species distributions (especially $\mathrm{CO})$ have also been used to constrain upper mesospheric wind patterns $(\sim 80-110 \mathrm{~km})($ e.g. Lellouch et al. 1997; Schubert et al. 2007). Ground-based measurements have also been used to monitor these time variable winds above the cloud tops (Clancy and Muhleman 1991; 
Fig. 18 Illustration of the major components of the global circulation of the Venus upper atmosphere. SS-AS (subsolar-to-antisolar), RSZ (retrograde superrotating zonal), ET (evening terminator), MT (morning terminator) (from Brecht et al. 2011)

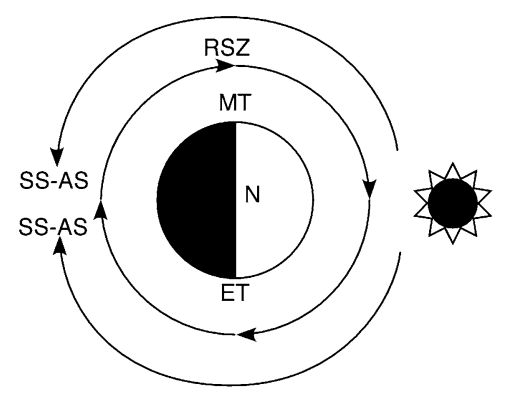

Table 1 Venus Middle-Upper Atmosphere Wind Proxies (Adapted from Schubert et al. 2007)

\begin{tabular}{|c|c|c|c|}
\hline Species/Emissions/Temperatures & $\begin{array}{l}\text { Altitude } \\
(\mathrm{km})\end{array}$ & $\begin{array}{l}\text { SS-AS Winds } \\
(\mathrm{m} / \mathrm{sec})\end{array}$ & $\begin{array}{l}\text { RSZ Winds } \\
(\mathrm{m} / \mathrm{sec})\end{array}$ \\
\hline Visible Spectroscopy (Solar Radiation) ${ }^{\mathrm{a}}$ & $\sim 70$ & & $83 \pm 27$ \\
\hline Temperatures (IR and Occultation) ${ }^{\mathrm{b}}$ & $70-90$ & & Variable (weak) \\
\hline $\mathrm{CO} \mathrm{mm}, \mathrm{CO}$ distribution $^{\mathrm{c}}$ & $\sim 80-100$ & Present & Variable \\
\hline $\mathrm{CO} \mathrm{mm}$, winds ${ }^{\mathrm{d}}$ & $\sim 90-105$ & $\leq 40-322 \pm 25$ & $35-147 \pm 3$ (variable) \\
\hline 10-micron, $\mathrm{CO}_{2}$ heterodyne $\mathrm{e}^{\mathrm{e}}$ & $109 \pm 10$ & $\leq 35-129 \pm 1$ & $3 \pm 7-40 \pm 3$ (weak) \\
\hline $\mathrm{O}_{2} \operatorname{IR}(1.27 \text { microns })^{\mathrm{f}}$ & $90-110$ & & Variable $(\sim 10-50)$ \\
\hline $\mathrm{O}_{2}$ Visible $(400-800 \mathrm{~nm})^{\mathrm{g}}$ & $100-130$ & & Weak $(\leq 30)$ \\
\hline CO 4.7-micron, winds ${ }^{\mathrm{h}}$ & $125-145$ & Sum $=200 \pm 50$ & \\
\hline NO nightglow (UV) ${ }^{\mathrm{i}}$ & $115-150$ & $\sim 200$ & $40-60$ \\
\hline O dayglow $(130 \mathrm{~nm})^{\mathrm{j}}$ & $130-250$ & & Eddy diffusion constraint \\
\hline Temperatures (night) ${ }^{\mathrm{k}}$ & Above 150 & $\sim 200$ & $\sim 50-100$ \\
\hline H dayglow $(121.6 \mathrm{~nm})^{1}$ & Above 150 & & $\sim 45-90$ \\
\hline $\mathrm{H}$ and $\mathrm{He}$ densities ${ }^{\mathrm{m}}$ & Above 150 & & $\sim 45-90$ \\
\hline
\end{tabular}

References: (a) Widemann et al. 2007; (b) Taylor et al. 1980; Schafer et al. 1990; Roos-Serote et al. 1995; Kliore 1985; (c) Gulkis et al. 1977; Schloerb et al. 1980; Clancy and Muhleman 1985, 1991; Gurwell et al. 1995; Lellouch et al. 1994; Rosenqvist et al. 1995; (d) Shah et al. 1991; Lellouch et al. 1994; Rosenqvist et al. 1995; Clancy et al. 2008; Lellouch et al. 2008; Rengel et al. 2008; (e) Goldstein et al. 1991; Schmülling et al. 2000; Sornig et al. 2008; (f) Crisp et al. 1996; Bougher et al. 1997; (g) Krasnopolsky 1983; Bougher and Borucki 1994; (h) Crovisier et al. 2006; (i) Stewart et al. 1980; Bougher et al. 1990; Gérard et al. 1981; (j) Alexander et al. 1993; (k) Niemann et al. 1980; Mayr et al. 1980; Mengel et al. 1989; Keating et al. 1980; Bougher et al. 1986, 1997; (l) Paxton 1985; Paxton et al., 1988a, 1988b; (m) Niemann et al. 1979; Brinton et al. 1980.

Lellouch et al. 1997; Schubert et al. 2007; Clancy et al. 2008). Many of these datasets, and their implications for global winds, are summarized in Table 1.

More recently, departures from this two-component wind model (residuals) are becoming more apparent, as ground-based and VEx measurements are being combined to confirm: (a) significant nightglow $\left(\mathrm{O}_{2}{ }^{1} \Delta \mathrm{g}\right.$ IR and VEx NO UV) temporal and spatial variability, and (b) a substantial warming (up to $\sim 180-195 \mathrm{~K}$ ) of mean temperatures near $\sim 95 \mathrm{~km}$ often associated with these $\mathrm{O}_{2}\left({ }^{1} \Delta_{\mathrm{g}}\right)$ nightglow patches of peak emission (e.g. Bertaux et al. 2007; Drossart et al. 2007; Bailey et al. 2008a; Ohtsuki et al. 2005, 2008). Systematic monitoring by other VEx instruments (since 2006) also provides confirmation of this proxy wind record, making use of vertical structure measurements. For example, VeRA and VIRTIS observations also address upper atmosphere dynamics by measuring the 3-D temperatures and de- 
riving the thermal wind fields ( 40-90 km) (Pätzold et al. 2007; Piccialli et al. 2008, 2012). Furthermore, SPICAV provides repeated measurements of vertical profiles of atmospheric density (and inferred temperatures) over $\sim 80-150 \mathrm{~km}$ (nightside) via stellar occultations (e.g. Bertaux et al. 2007; Piccialli et al. 2015). These combined nightglow and vertical structure measurements are generally consistent with weak RSZ winds (on average) above $\sim 80-90 \mathrm{~km}$, with RSZ winds increasing in magnitude (on average) above $\sim 115-120 \mathrm{~km}$.

The large variability of the RSZ winds between $\sim 80$ and $110 \mathrm{~km}$ has been the subject of considerable debate. Some investigators have attributed this variability to the changing nature of gravity wave breaking in this region (Alexander 1992; Zhang et al. 1996; Bougher et al. 1997, 2006; Zalucha et al. 2013). Alternatively, upward propagating tides and planetary scale waves may serve to significantly modify the density and wind structure of the Venus upper atmosphere, analogous to that which has been observed at Mars (Forbes and Hagan 2000; Forbes and Konopliv 2007). Nevertheless, the processes responsible for maintaining (and driving variations in) the SS-AS and RSZ winds in the Venus upper atmosphere are still not well understood or quantified (e.g. Bougher et al. 2006).

\subsection{Variability in the Upper Mesosphere from NLTE Observations}

The key transition region between the upper mesosphere and the thermosphere is traditionally characterized by the coldest atmospheric region, the mesopause, and in the case of Venus, with a nearby feature sometimes called "mesopeak" (see Fig. 14), whose observational characterization is far from complete (Clancy et al. 2003). Since the early non-LTE works of Dickinson in the early 70s this warm layer is expected to respond to a direct solar absorption by $\mathrm{CO}_{2}$ in the near-IR, i.e. it is a specific daytime feature (Dickinson 1972; Bougher and Roble 1991; Roldan et al. 2000). Information about the strength and variability of this warming is still needed in order to clarify the role of dynamics at these altitudes and validate the non-LTE models.

Unfortunately, the temperatures from retrievals and models presented in Sect. 3.6 do not completely clarify this issue. They show an agreement between VIRTIS observations and the VTGCM at high latitudes, with a clear mesopeak around $115 \mathrm{~km}$, but a significantly colder upper mesosphere in the VIRTIS data than in the model, with no mesopeak (within the limitations imposed by the large VIRTIS error bars). This seems to be in contradiction with the GCM and with the simpler 1-D radiative equilibrium models' predictions of a local maximum in temperature around the subsolar point (Bougher and Roble 1991; Roldan et al. 2000). One important aspect is that both $\mathrm{CO}$ and temperature come from the same VIRTIS dataset and should be explained simultaneously, which currently represents a challenge. The global CO results from VIRTIS, however, are consistent with a CO distribution controlled by dynamics below about $130 \mathrm{~km}$ at least, and similar sub-solar to anti-solar variations are found with the VTGCM although the VIRTIS CO densities are systematically larger. Whether the two discrepancies, in temperature and in $\mathrm{CO}$, are somehow related or they point to deficiencies in the data and/or in the models has not been determined.

\section{Three-Dimensional Modeling}

Three-dimensional modeling tools continue to be used to study the Venus upper atmosphere circulation and the resulting tracer emissions, species, and temperature distributions (see reviews by Bougher et al. 1997, 2006; more recently see Brecht et al. 2011; Tingle 2011; Brecht and Bougher 2012; Hoshino et al. 2012). GCMs are crucial to synthesize the various 
density, temperature, and airglow datasets for extracting SS-AS and RSZ wind components above the cloud tops. Nevertheless, GCM models to date are generally designed as climate models, and are best compared with statistically averaged maps of nightglow and species distributions, as well as averaged temperature structures. Furthermore, existing GCMs are presently unable to reproduce the observed large density, temperature, and airglow variability (especially over $\sim 70-120 \mathrm{~km}$ ). Recent GCM studies employing gravity, tidal, and planetary wave forcing schemes are beginning to address this variability.

The Venus Thermospheric General Circulation Model (VTGCM) is one such upper atmosphere GCM that will be described in detail below. The strategy for use of the VTGCM has been twofold: (a) to compare the climate averaged circulation and resulting density, nightglow and temperature distributions with statistically averaged maps of corresponding measured quantities of the mesosphere-thermosphere of Venus; and (b) to subsequently drive variations in the VTGCM structure and winds using existing gravity wave, tidal, and planetary wave forcing schemes. This two-fold strategy is designed to capture the statistically average behavior of the global wind patterns first (and the corresponding density, temperature and nightglow distributions), before attempting to simulate the variability that is strongly evident in these same fields over $\sim 70-120 \mathrm{~km}$. This strategy offers a systematic method to progress beyond a climate model treatment of the Venus upper atmosphere, and thereby uncover the responsible processes controlling the upper atmosphere structure and global circulation patterns and their variability.

\subsection{VTGCM Framework, Inputs, and Tunable Parameters}

The VTGCM is a 3-D finite difference hydrodynamic model of Venus' upper atmosphere (e.g. Bougher et al. 1988) which is based on the National Center for Atmospheric Research terrestrial Thermospheric General Circulation Model (TGCM). The VTGCM has been thoroughly documented as it has been revised and improved over the last two decades; presented in this section is an overview of the model as implemented recently in the revised version of Brecht et al. (2011) and Brecht and Bougher (2012). The VTGCM solves the time-dependent primitive equations for the neutral upper atmosphere. The diagnostic equations (hydrostatic and continuity) provide geopotential and vertical motion fields. Additionally, the prognostic equations (thermodynamic, eastward and northward momentum, composition) are typically solved for steady-state solutions for the temperature, zonal and meridional velocity, and the mass mixing ratios of specific species. The VTGCM model domain covers a $5^{\circ}$ by $5^{\circ}$ latitude-longitude grid, with 69 evenly spaced log-pressure levels in the vertical, extending from $\sim 70$ to $300 \mathrm{~km}(\sim 70$ to $200 \mathrm{~km}$ ) at local noon (midnight). This altitude range ensures that all dynamical influences contributing to the nightglow layers (i.e. $\mathrm{NO}, \mathrm{O}_{2}, \mathrm{OH}$ ) can be captured, and wave propagation above the cloud tops can be addressed. The lower boundary of the VTGCM has recently been improved to include the self-consistent latitude and local time variation of temperatures, zonal and meridional winds, and heights near $\sim 69 \mathrm{~km}$, in accord with current Venus lower atmosphere General Circulation Models and available VIRA datasets (Kliore et al. 1985).

The VTGCM includes parameterizations for $\mathrm{CO}_{2} 15-\mu \mathrm{m}$ cooling, infrared (IR) heating, and extreme ultraviolet (EUV) heating. Reference $\mathrm{CO}_{2} 15-\mu \mathrm{m}$ cooling rates for a given temperature and composition profile are taken from Roldan et al. (2000); cooling rates for the simulated VTGCM temperatures and species abundances are calculated (from these reference rates) based upon a slight modification of the parameterization scheme described previously (e.g. Bougher et al. 1986). The near-IR heating term is incorporated in the VTGCM using an online simulated look-up table (organized as a function of pressure and SZA), and 
updated recently using Roldan et al. (2000) rates. The VTGCM can capture the full range of EUV-UV flux conditions. The latest ion-neutral reactions and rates being used in the VTGCM are largely taken from Fox and Sung (2001).

Sub-grid scale wave effects cannot be captured by the VTGCM directly. Instead, wave drag is presently being prescribed as a Rayleigh friction term within the VTGCM in order to mimic first order wave-drag effects on the mean flow (Bougher et al. 1999). Detailed gravity wave momentum deposition schemes have been tested for replacement of Rayleigh friction within the VTGCM code (e.g. Zalucha et al. 2013). However, such schemes are not presently validated for dedicated usage in VTGCM simulations. The eddy diffusion coefficient is prescribed using a common aeronomical formulation (see von Zahn et al. 1979). The nightside eddy diffusion coefficient has a prescribed maximum value of $\sim 1.0 \times 10^{7} \mathrm{~cm}^{2} \mathrm{~s}^{-1}$ (see Brecht et al. 2011), approximately 5-10 times the dayside value typically used. This nightside value is commonly varied in sensitivity studies.

\subsection{VTGCM Outputs and Comparisons with Various Vex Datasets}

It is noteworthy that the VTGCM has largely been run as a climate model thus far. This means that model adjustable parameters have been specified to enable time and spatially averaged $\mathrm{VEx} \mathrm{O}_{2}$ and NO nightglow distributions (Brecht et al. 2011), VEx atomic O density distributions (Brecht et al. 2012), VEx and ground-based mean dayside temperatures (Brecht and Bougher 2012), and time-averaged terminator temperature profiles (Bougher et al. 2015) to be reproduced by corresponding VTGCM outputs. Nevertheless, the changing SS-AS and RSZ wind components of the real atmosphere provide significant variations of these densities, temperatures, and nightglow distributions over time, for which the climate model capability of the VTGCM is not adequate to address.

Figure 19 illustrates the VTGCM "best case" neutral temperatures near the equator for solar minimum conditions (appropriate to early Venus Express sampling). These results (and those presented in Figs. 20, 21, 23, and 24) were obtained following the model run described in Bougher et al. (2015). Dayside temperatures approach $\sim 240-245 \mathrm{~K}$ above $\sim 160 \mathrm{~km}$, in accord with previous estimates for solar minimum conditions (e.g. Kasprzak et al. 1997). A mesopeak of warm temperatures is also simulated near $\sim 115 \mathrm{~km}$, in accord with previous 1-D radiative equilibrium calculations (Roldan et al. 2000) and ground-based measurements at $\sim 110 \mathrm{~km}$ (Sonnabend et al. 2010). The VTGCM calculation also shows a peak nightside temperature of $\sim 195 \mathrm{~K}$ at $\sim 103 \mathrm{~km}$. This warm temperature, absent in earlier versions of the VTGCM, is directly connected with the more realistic $4.3-\mu \mathrm{m}$ heating on the dayside, and the resultant enhancement of day-to-night winds in the lower thermosphere. The stronger winds create a larger thermal advection and result in increased adiabatic heating near midnight. The VTGCM nightside temperature of $\sim 195 \mathrm{~K}$ near $\sim 100 \mathrm{~km}$ corresponds to mean wind conditions, in accord with statistically averaged VEx spacecraft and groundbased observations (e.g. Bertaux et al. 2007; Bailey et al. 2008a, 2008b; Clancy et al. 2008; Brecht and Bougher 2012).

Figure 20 illustrates 1-D heat balance terms for an overhead sun $\left(\mathrm{SZA} \sim 0^{\circ}\right)$ from the same VTGCM "best case". Notice that at the peak of EUV heating $(\sim 150-160 \mathrm{~km})$, the primary cooling agent is $\mathrm{CO}_{2} 15-\mu \mathrm{m}$ cooling. This balance determines that the solar cycle variation of topside temperatures for Venus is rather small $(\sim 60-70 \mathrm{~K})$ (see Kasprzak et al. 1997). Below $140 \mathrm{~km}$, near IR heating is primarily balanced by $\mathrm{CO}_{2} 15-\mu \mathrm{m}$ cooling. Dynamical terms appear to play a small role on Venus dayside thermosphere (i.e. a nearly radiative balance is achieved).

Figure 21 shows zonal wind profiles corresponding to the same "best case" simulation at the equator for VTGCM morning terminator (MT) and evening (ET) terminator winds. 


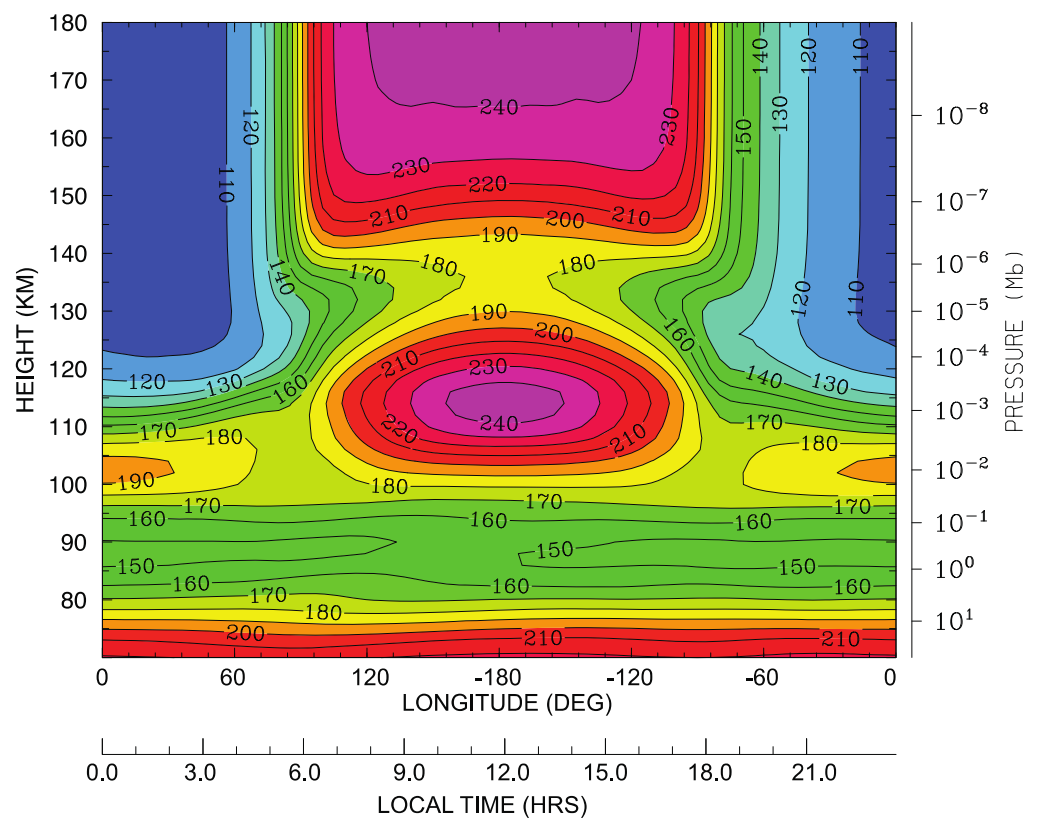

Fig. 19 VTGCM slice (70-180 km) near the equator for neutral temperatures (K) (altitude vs. LT). Solar minimum conditions (F10.7 70 at Earth) are utilized (from Bougher et al. 2013)

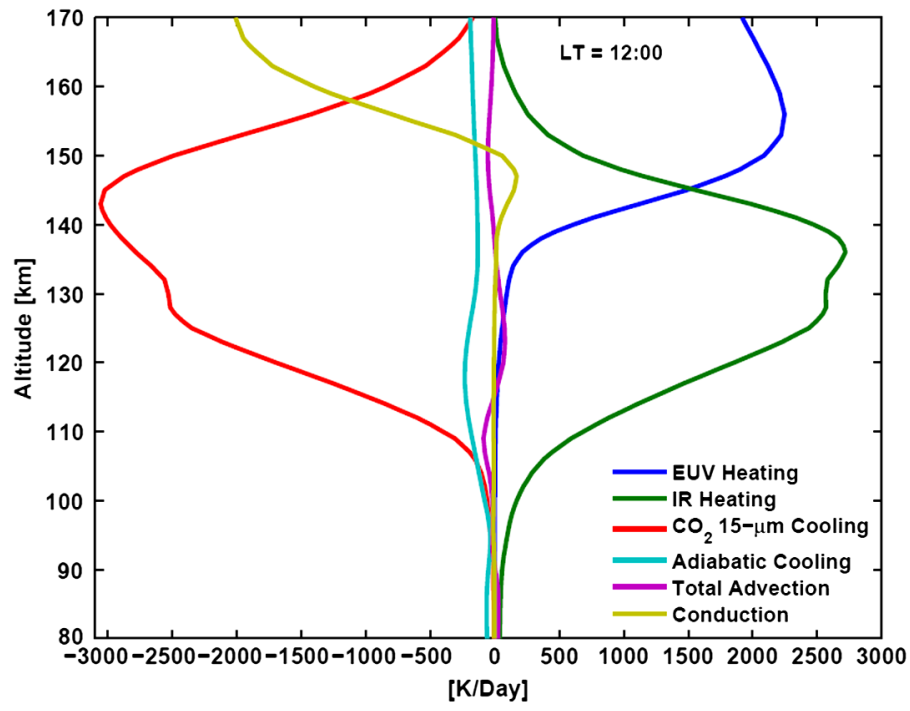

Fig. 20 VTGCM heating and cooling rates (K/day) for solar minimum conditions at SZA $\sim 0^{\circ}$ (on the equator at noon) (from Brecht and Bougher 2012)

These panels clearly indicate that symmetric (SS-AS only) winds have equal (but opposite signed) magnitudes at the ET and MT locations, while asymmetric (SS-AS + RSZ) winds do not. The difference between these two components is plotted in the last panel, illustrating 
Fig. 21 VTGCM profiles at the equator for morning (MT) and evening (ET) terminator winds $(\mathrm{m} / \mathrm{s})$. Symmetric winds (dotted lines) have no RSZ component, while asymmetric winds (solid lines) do. Winds are positive from West to East (from Brecht and Bougher 2012 and Bougher et al. 2013)

Fig. 22 Morning terminator (top) and evening terminator (bottom) panels illustrate temperature profiles from both VEx SOIR measurements and various VTGCM simulations for the $30-60^{\circ} \mathrm{N}$ latitude bin. Simulations refer to: (a) solar minimum (SMIN) conditions (blue), (b) solar moderate (SMOD) conditions (green), (c) SMIN with maximum wave drag (red), and (d) SMIN with minimum wave drag (light blue). The SOIR VAST averaged profile is shown for comparison (purple). The left vertical axis throughout is given in mbars (from Bougher et al. 2015)
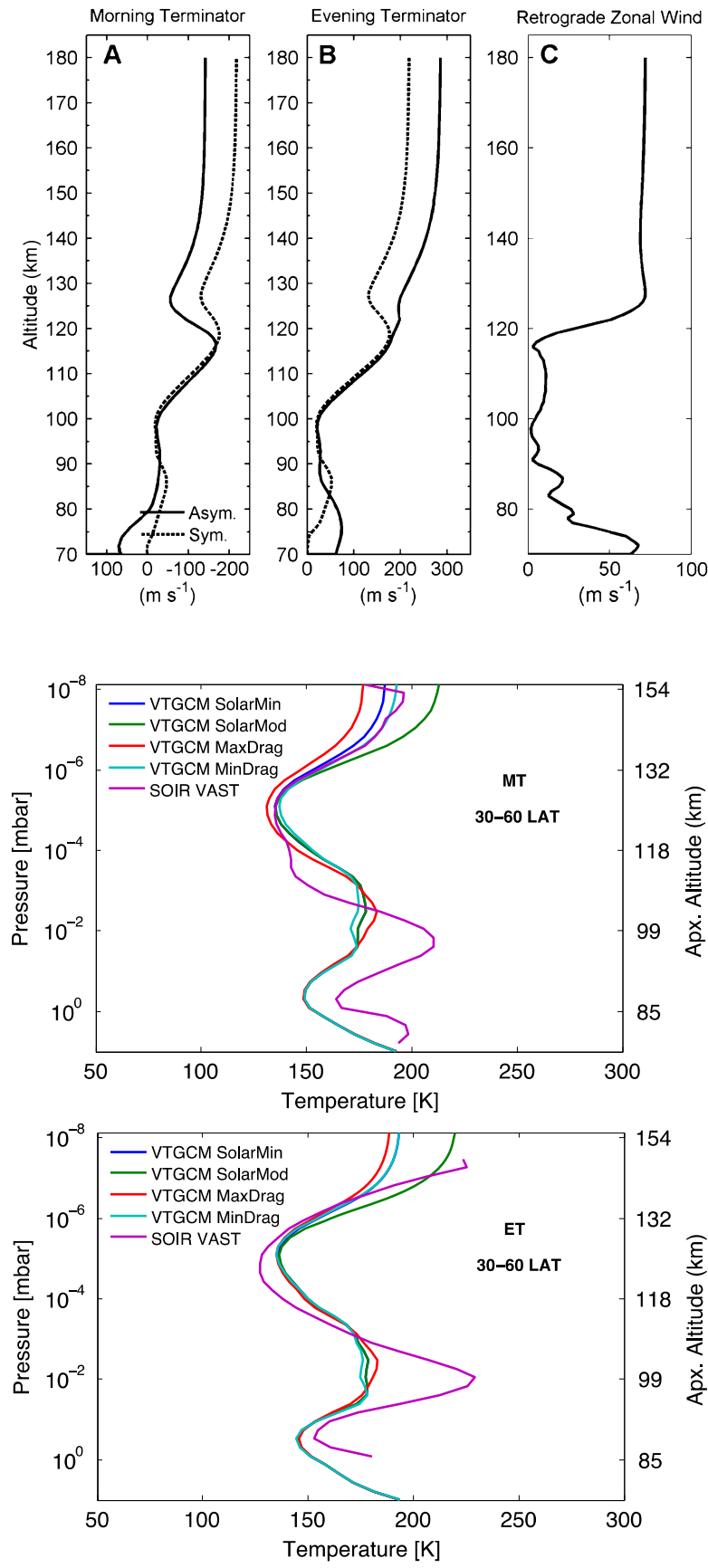

the fact that VTGCM prescribed RSZ winds are very weak from $\sim 90 \mathrm{~km}$ to $115 \mathrm{~km}$. This prescribed behavior is physically based upon statistically averaged VEx measurements and their implications for altitude varying zonal winds. Above $\sim 115 \mathrm{~km}, \mathrm{RSZ}$ winds are increasing yielding values that approach $\sim 70-80 \mathrm{~m} \mathrm{~s}^{-1}$ (see Brecht and Bougher 2012 and Bougher 

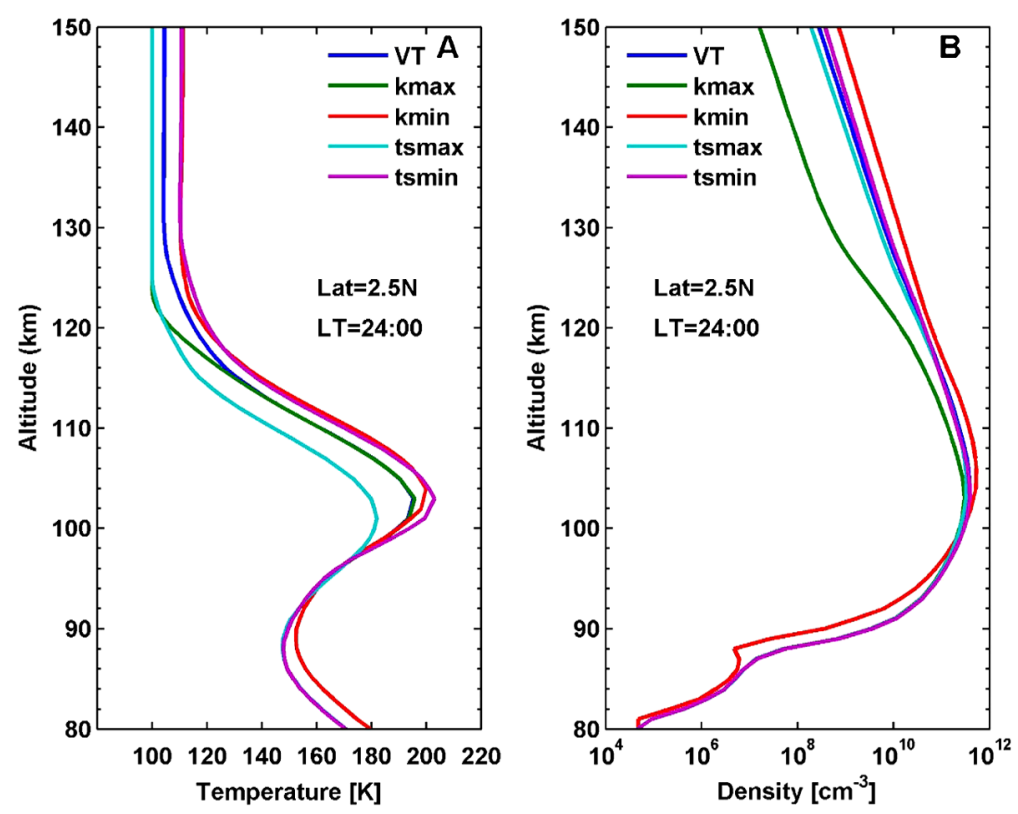

Fig. 23 VTGCM temperature and O density profiles (midnight at the equator) for standard (blue curve) and extreme values of the nightside eddy diffusion coefficient (kmax, kmin) and wave drag parameter (tsmax, tsmin). Variations in temperatures at 100-105 km are large (180-205 K). Corresponding variations in $\mathrm{O}$ densities are large above $\sim 110 \mathrm{~km}$ (from Bougher et al. 2013). Only one variable at a time is varied for each simulated profile (the other being held fixed)

et al. 2013). These RSZ wind magnitudes above $\sim 130 \mathrm{~km}$ are consistent with NO nightglow statistically averaged distributions gleaned from existing PVO and VEx measurements.

The dayside to nightside transition at the ET and MT should yield temperature profiles that are consistent with the combined SS-AS and RSZ circulation patterns; see the zonal wind profiles shown in Fig. 21. A comparison of terminator temperature profiles is given in Fig. 22, for which VEx temperature profiles deduced from SOIR are plotted against various model runs of the VTGCM. VTGCM simulated temperatures reasonably match SOIR profiles above $\sim 105-110 \mathrm{~km}$; below, simulated temperature values are much cooler, indicating a missing VTGCM heating source, especially at low latitudes (Bougher et al. 2015). MT profiles match best above $\sim 100 \mathrm{~km}$; however, at lower altitudes, SOIR temperatures are much warmer than VTGCM simulations.

Sensitivity tests were also performed to investigate the role of eddy diffusion and winds in driving variability in the VTGCM (e.g. Brecht et al. 2011). These tests revealed the wave drag parameter (impacting wind magnitudes) controls the intensity of the nightglow emissions, while eddy diffusion (impacting vertical mixing) controls the altitude of the nightglow layers. Figure 22 shows midnight profiles of: (a) temperatures and (b) $\mathrm{O}$ densities for the "best case" VTGCM simulation, along with profiles for extreme values of the nightside eddy coefficient $\left(\mathrm{K}_{\min }=0.1 \times 10^{7} \mathrm{~cm}^{2} \mathrm{~s}^{-1}\right.$ and $\left.\mathrm{K}_{\max }=8 \times 10^{7} \mathrm{~cm}^{2} \mathrm{~s}^{-1}\right)$ and the wave drag parameter (Rayleigh friction timescale: $\tau_{\min }=0.7 \times 10^{-4} \mathrm{~s}^{-1}$ and $\tau_{\max }=2.0 \times 10^{-4} \mathrm{~s}^{-1}$ ). The large variations in $\mathrm{O}$ densities give rise to a factor of $\sim 2.5$ change in the peak $\mathrm{O}_{2}$ IR nightglow intensities ( $\sim 1.4$ to $3.3 \mathrm{MR})$. Temperatures also change dramatically near $\sim 100$ $105 \mathrm{~km}$, in accord with VEx and ground-based measurements (e.g. Bertaux et al. 2007; Bailey et al. 2008a; Ohtsuki et al. 2008). 


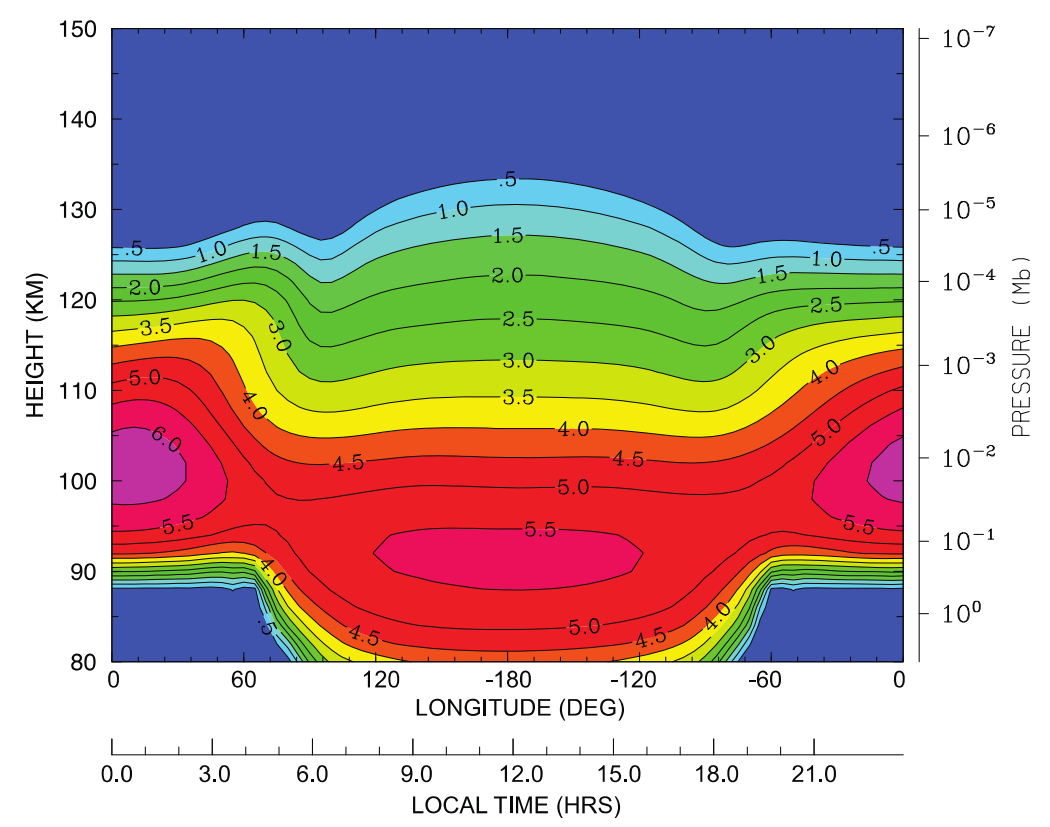

Fig. 24 The $\mathrm{O}_{2}$ nightglow peak volume emission rate $\left(\log 10, \mathrm{ph} / \mathrm{cm}^{3} \mathrm{~s}^{-1}\right)$ at the equator spanning 80-180 km. The VTGCM produces $\mathrm{O}_{2}$ IR nightglow peak intensities within the observational ranges provided by VEx as described in Gérard et al. (2008b, 2009a), and is in accord with hemispheric mean values derived in those studies (from Bougher et al. 2013)

The VTGCM "best case" also produces $\mathrm{O}_{2}$ IR nightglow peak intensities within the observational ranges provided by VEx as described in Gérard et al. (2008c, 2009b), and is in accord with hemispheric mean values derived in those studies. The $\mathrm{O}_{2}$ nightglow peak volume emission rate (Fig. 24) produced by the VTGCM is $\sim 2.1 \times 10^{6}$ photon $\mathrm{cm}^{-3} \mathrm{~s}^{-1}$ and is located near the equator at 00:00 LT at an altitude of $\sim 101 \mathrm{~km}$ (close to the warm region in Fig. 23). The corresponding $\mathrm{O}_{2}$ nightglow peak vertical intensity was calculated to be $\sim 2.12 \mathrm{MR}$. Optical depth effects are not important for this nightside emission. Statistically averaged observations reveal the mean peak intensity to be $\sim 1.9 \mathrm{MR}$ at $96 \pm 2.7 \mathrm{~km}$ and on average appearing near midnight (Gérard et al. 2008c; Soret et al. 2012a). The VTGCM produces a hemispheric average intensity of $\sim 0.53 \mathrm{MR}$, which is in excellent agreement with the similarly calculated VEx value of $\sim 0.50 \mathrm{MR}$ (e.g. Soret et al. 2012a). The corresponding nightside VTGCM O density map (Brecht et al. 2012) is also in accord with the VEx deduced map (Soret et al. 2012a) within $\sim 30-45^{\circ}$ of the anti-solar point.

The VTGCM produces NO UV nightglow (both peak and hemispheric mean) vertical intensities slightly smaller than the observational ranges provided by VEx as described in Gérard et al. (2008b), Cox et al. (2010) and Stiepen et al. (2013). The NO nightglow peak volume emission rate (Fig. 25) produced by the VTGCM is $\sim 1.4 \times 10^{3}$ photon $\mathrm{cm}^{-3} \mathrm{~s}^{-1}$ and is located near the equator at 01:00 LT at an altitude of $\sim 108-109 \mathrm{~km}$. The corresponding NO nightglow peak vertical intensity was calculated to be $\sim 2.2 \mathrm{kR}$. Statistically averaged observations reveal the mean peak intensity to be $\sim 4.0 \mathrm{kR}$ at $115 \pm 7 \mathrm{~km}$ and on average appearing near 2-3 AM (Stewart et al. 1980; Stiepen et al. 2012, 2013). The VTGCM produces a hemispheric average intensity of $\sim 1.0 \mathrm{kR}$, which is about $50 \%$ of the similarly 


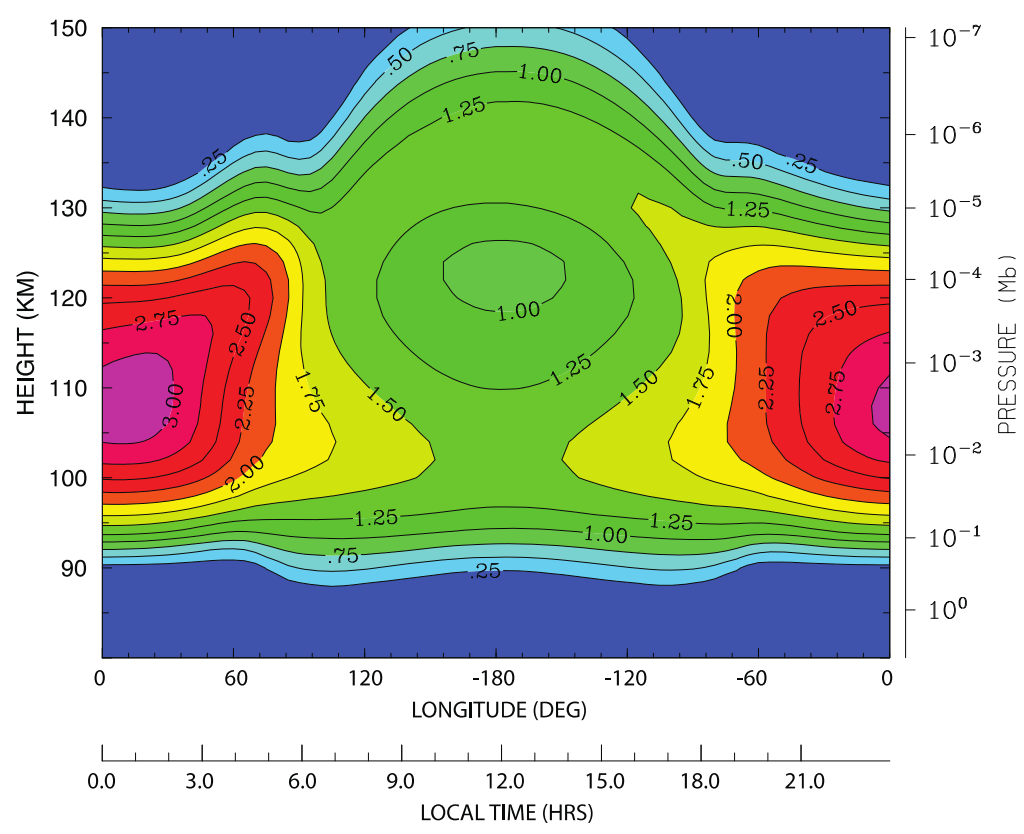

Fig. 25 The NO nightglow peak volume emission rate $\left(\log 10 \mathrm{ph} / \mathrm{cm}^{3} / \mathrm{s}\right)$ at the equator spanning $80-160 \mathrm{~km}$. The simulated NO UV nightglow peak volume emission rate is $\sim 1.4 \times 10^{3}$ photon $\mathrm{cm}^{-3} \mathrm{~s}^{-1}$ and is located near the equator at 01:00 LT at an altitude of $\sim 108-109 \mathrm{~km}$. The corresponding NO nightglow peak vertical intensity was calculated to be $\sim 2.2 \mathrm{kR}$ (from Bougher et al. 2013)

calculated VEx value of $\sim 1.9 \mathrm{kR}$ (e.g. Stiepen et al. 2013). In addition, the VTGCM simulations of the NO nightglow layer provide peak intensities ( $\sim 50 \%$ of those observed) and peak layer altitudes $(\sim 6 \mathrm{~km}$ lower than observed) that are not consistent with corresponding 1-D models (e.g. Stiepen et al. 2013). Differences in 1-D versus 3-D model treatments of prescribed eddy diffusion coefficients are being investigated to potentially raise the simulated airglow layer, increase the resulting vertical intensities toward observed values, and move the location of the statistical peak emission toward 3 AM.

\subsection{LMD-VGCM Model and O Density}

The LMD Venus General Circulation Model (LMD-VGCM) (Lebonnois et al. 2010) has been extended by Gilli et al. (2017) to include thermospheric dynamics and photochemistry. The model extends from the ground up to the $\sim 150 \mathrm{~km}$ level and solves the primitive equations of hydrodynamics. The photochemical module includes 34 species providing a full description of the chemistry of carbon dioxide, oxygen, hydrogen, chlorine and sulfur compounds implying 102 reactions. Non-orographic gravity waves are generated above the middle cloud region, propagate upward and break in the thermosphere. They described the processes occurring in the 'transition' region between the zonal circulation and the subsolar to antisolar flow. The atomic oxygen concentrations predicted by the LMD-VGCM peak around $100 \mathrm{~km}$ at nighttime. They were compared with the $\mathrm{O}$ density profile derived by Gérard et al. (2009a) and Soret et al. (2012a) from the VIRTIS $\mathrm{O}_{2} 1.27 \mu \mathrm{m}$ nightglow observations, the VTGCM results by Brecht et al. (2012) and the photochemical model by Krasnopolsky (2013). The agreement was generally good except near the anti-solar point 
where the LMD-VGCM densities are larger than observed by a factor $\sim 2$. Initial comparisons of LMD-VGCM and VTGCM upper atmosphere fields have been conducted, most recently making use of VIRTIS CO density and temperature profiles spanning $\sim 100$ to $150 \mathrm{~km}$ (Gilli et al. 2015). See Sect. 3.6.1.

\subsection{Gravity Waves: Airglow and Other Signatures}

There is abundant evidence for waves near Venus cloud tops (e.g. Schubert 1983; Gierasch et al. 1997) and at thermosphere heights (e.g. Kasprzak et al. 1993; Bougher et al. 1997). For instance, UV features interpreted as convective cells (Del Genio and Rossow 1982; Titov et al. 2012) with horizontal scales of hundreds of $\mathrm{km}$ (gravity waves) have been observed. The VEGA balloons in 1985 (Linkin et al. 1986) probed the unstable layer at about $50-55 \mathrm{~km}$ and found vertical velocities of $\sim 1 \mathrm{~m} / \mathrm{s}$ associated with convective motion. Recently, perturbations in density, temperature, airglow, and cloud structures were observed by VEx, putatively caused by gravity waves in Venus' upper atmosphere. For example, VIRTIS has detected perturbations in $\mathrm{CO}_{2}$ NLTE $4.3 \mu \mathrm{m}$ emissions (Garcia et al. 2009), and both VIRTIS and VMC observed visible trains of oscillating cloud brightness in the UV for an upper cloud layer $(\sim 66 \mathrm{~km})$ on the dayside and from thermal radiation from the lower cloud layer on the nightside (e.g. Peralta et al. 2008; Titov et al. 2012; Piccialli et al. 2015). The VeRa Radio Science Experiment also provides wave information that is extracted from temperature fluctuations (Tellmann et al. 2012). Lastly, current efforts are focusing upon characterizing gravity waves from VIRTIS observations of $\mathrm{O}_{2}$ IR nightglow (Altieri et al. 2014).

The importance of planetary scale waves in the Venus atmosphere at/above the cloud tops has also been investigated for many years (see Schubert 1983; Schubert et al. 2007). For instance, Del Genio and Rossow (1990) analyzed Pioneer Venus cloud brightness data and showed the dominance of a wave that rotates around Venus in the equatorial region with a period of about 4 days. This wave was identified as a Kelvin wave. Del Genio and Rossow (1990) also reported the dominance of the 5-day period wave at mid-latitudes $\left(\sim 45^{\circ}\right.$ latitude) at the cloud tops, and suggested that this 5-day wave would be a Rossby wave. Furthermore, Rossow et al. (1990) determined that the zonal wind fluctuations associated with diurnal and semi-diurnal tides are approximately $10 \mathrm{~m} / \mathrm{s}$ at the cloud tops. Pechmann and Ingersoll (1984) estimated the vertical wavelengths of these upward propagating tides to be approximately 7 and $30 \mathrm{~km}$, respectively. Forbes and Konopliv (2007) suggested that planetary scale waves originating in the cloud layer would propagate to the thermosphere, based on an analysis of data obtained by Magellan. Recent GCM calculations by Hoshino et al. (2012) and Nakagawa et al. (2013) further suggest that only Kelvin waves propagate up to thermospheric altitudes (up to $\sim 130 \mathrm{~km}$ ) with significant amplitudes, and not tides. Rossby waves have small amplitudes at $\sim 130 \mathrm{~km}$. The presence of these planetary waves at the cloud level was also observed using VEx instruments (e.g. Titov et al. 2012).

\section{Hot Atoms and Escape Processes}

Evidence for the presence of hot $\mathrm{H}$ and $\mathrm{O}$ atoms in the upper thermosphere, their sources and their escape processes have been reviewed by Nagy et al. (1990), Shizgal and Arkos (1996), Fox and Hać (1997), Lichtenegger et al. (2009) and Gröller et al. (2010). The presence of hot $\mathrm{H}$ atoms in the Venus exosphere was discovered during the Mariner-5 and 10 missions (Anderson 1976) and the existence of a hot O population (Nagy et al. 1981) was identified 
with the PV-OUVS instrument. More recently, observations of hot $\mathrm{H}$ have been made with SPICAV. In this section, we first review recent results related to observations and modeling of hot $\mathrm{H}$ and $\mathrm{O}$ atoms. We then summarize the processes leading to escape of $\mathrm{H}$ and $\mathrm{O}$ atoms.

\subsection{Sources and Distribution of Hot $\mathrm{H}$ and $\mathrm{O}$ Atoms}

In the Venus upper atmosphere, nonthermal (hot) atoms are created by exothermic ionneutral reactions. Unlike in the lower thermosphere where newly created hot atoms are quickly thermalized by collisions, in the upper thermosphere and in the exosphere where collisions become less frequent, these hot atoms are not fully thermalized and form extended coronae of $\mathrm{H}$ and $\mathrm{O}$ atoms above the exobase located near $200 \mathrm{~km}$ where collisions become infrequent.

\subsubsection{Hot H Atoms}

Charge exchange and momentum transfer collisions between $\mathrm{H}$ and ionospheric $\mathrm{H}^{+}$and $\mathrm{O}^{+}$are probably a major sources of the hot hydrogen population (Hodges 1999) initially observed by Mariner 5 and 10:

$$
\begin{aligned}
& \mathrm{H}^{+}+\mathrm{H} \rightarrow \mathrm{H}^{*}+\mathrm{H}^{+} \\
& \mathrm{H}^{+}+\mathrm{O} \rightarrow \mathrm{H}^{*}+\mathrm{O}^{+} \\
& \mathrm{O}^{*}+\mathrm{H} \rightarrow \mathrm{H}^{*}+\mathrm{O}
\end{aligned}
$$

Consequently, a large fraction of the hot $\mathrm{H}$ atoms produced in these reactions can overcome the escape energy for atomic hydrogen of about $0.56 \mathrm{eV}$. Observations of the hydrogen corona made by SPICAV on the dayside at altitudes from $1000 \mathrm{~km}$ to $8000 \mathrm{~km}$ were analyzed by Chaufray et al. (2012b). The derived density of hot hydrogen was very sensitive to the subtraction of interplanetary emission. The Lyman- $\alpha$ brightness profiles were modeled by a two-component $\mathrm{H}$ atom population (Fig. 26). The first one is a cold hydrogen population which dominates below $\sim 2000 \mathrm{~km}$ and the second one a hot hydrogen population which is dominant above $\sim 4000 \mathrm{~km}$. The temperature and hydrogen density at $250 \mathrm{~km}$ near noon of the cold populations are $\sim 300 \mathrm{~K}$ and $\sim 1 \times 10^{5} \mathrm{~cm}^{-3}$ respectively, in good agreement with previous observations. The transition between the cold and the hot population is sharp but variable with local time. A larger exobase density on the dawn side than on the dusk side was observed, consistent with the asymmetry previously observed, but with a lower dawn/dusk contrast. The night/day ratio was estimated $\sim 20$, a value smaller than the one deduced from ions measurements by the Pioneer Venus Orbiter. This smaller ratio could indicate a lower day/night temperature ratio during Vex conditions than PVO conditions. The density of the hot population was well reproduced by the exospheric model from Hodges (1999).

\subsubsection{Hot O Atoms}

The presence of corona of hot $\mathrm{O}$ atoms has been established on the basis of the data collected with the PV-OUVS instrument (Nagy et al. 1981; Nagy and Cravens 1988) and the Venera 11 mission (Bertaux et al. 1981). They derived a hot $\mathrm{O}$ density on the order of $10^{4} \mathrm{~cm}^{-3}$ at $1000 \mathrm{~km}$ (Fig. 27). Exothermic reactions such as electron dissociative recombination of $\mathrm{O}_{2}^{+}$ions are the main source of suprathermal $\mathrm{O}$ atoms in the upper atmosphere of Venus (Nagy et al. 1981; McElroy et al. 1982; Krestyanikova and Shematovich 2006; Lichtenegger 
Fig. 26 Lyman- $\alpha$ intensity profile observed with SPICAV (stars) and comparison with the best fit profile ( $T=271 \mathrm{~K}$ ) to the measurements below $2000 \mathrm{~km}$ (solid line). Intensity profiles at $241 \mathrm{~K}$ and $315 \mathrm{~K}$ are shown in blue and red, respectively. The scale height of the observed profile is much brighter than the best fit above $4000 \mathrm{~km}$ as a consequence of the presence of hot $\mathrm{H}$ atoms (from Chaufray et al. 2012b)

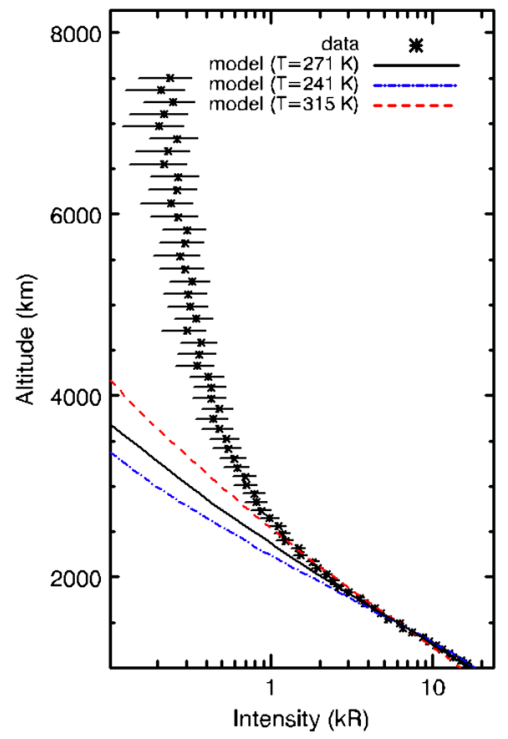

Fig. 27 density distribution of hot $\mathrm{O}$ atoms derived from the PV-OUVS observations and comparison with model calculations for low solar activity level (from Gröller et al. 2010)

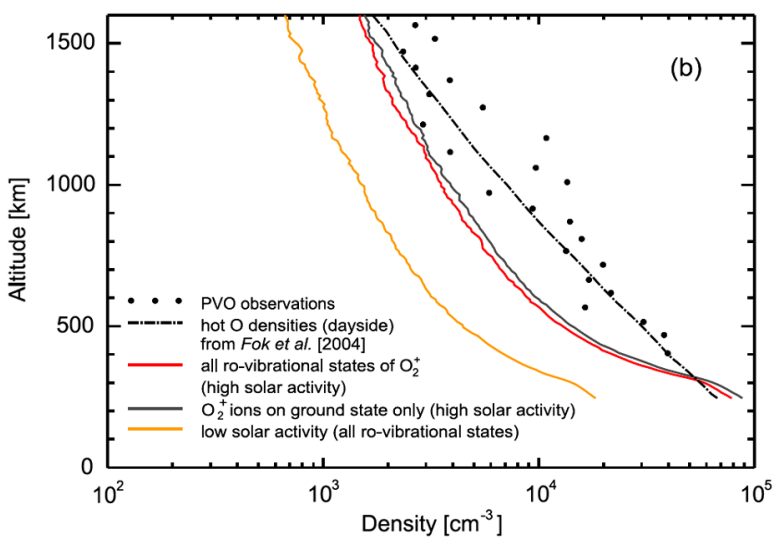

et al. 2009; Gröller et al. 2010, 2012). This process can proceed according to a several energetically allowed channels, with exothermicities:

$$
\begin{aligned}
& \mathrm{O}_{2}^{+}+\mathrm{e} \rightarrow \mathrm{O}\left({ }^{3} \mathrm{P}\right)+\mathrm{O}\left({ }^{3} \mathrm{P}\right)+6.99 \mathrm{eV} \\
& \mathrm{O}_{2}^{+}+\mathrm{e} \rightarrow \mathrm{O}\left({ }^{1} \mathrm{D}\right)+\mathrm{O}\left({ }^{3} \mathrm{P}\right)+5.02 \mathrm{eV} \\
& \mathrm{O}_{2}^{+}+\mathrm{e} \rightarrow \mathrm{O}\left({ }^{1} \mathrm{D}\right)+\mathrm{O}\left({ }^{1} \mathrm{D}\right)+3.06 \mathrm{eV} \\
& \mathrm{O}_{2}^{+}+\mathrm{e} \rightarrow \mathrm{O}\left({ }^{1} \mathrm{D}\right)+\mathrm{O}\left({ }^{1} \mathrm{~S}\right)+0.83 \mathrm{eV}
\end{aligned}
$$

where the branching ratios for the vibrational ground state $\mathrm{O}_{2}^{+}$at a zero collisional energy are also given in parentheses (Kella et al. 1997). Among these possible reaction paths, even the most exoenergetic channel provides only $63 \%$ of the escape velocity. Consequently, photochemical sources play an insignificant role in controlling the $\mathrm{O}$ escape rate from Venus. On the nightside, the $\mathrm{O}_{2}^{+}$ion density is low and hot atoms are also formed by charge exchange 
reactions:

$$
\begin{aligned}
& \mathrm{O}^{+}+\mathrm{H} \rightarrow \mathrm{O}+\mathrm{H}^{+} \\
& \mathrm{O}^{+}+\mathrm{O} \rightarrow \mathrm{O}^{+}+\mathrm{O}
\end{aligned}
$$

According to current models (Hodges 2000; Krestyanikova and Shematovich 2006; Gröller et al. 2010, 2012), these reactions produce a hot oxygen population. Stochastic models could reproduce the O density profiles reported by Nagy et al. (1981). These results depend on the input data, the numerical values of the parameters used and assumptions made in the calculations. Uncertainties in the parameters and simplifications can give rise to a wide range of possible results. Therefore, more realistic assumptions and accurate parameters may lead to lower densities than predicted by earlier models. Gröller et al. (2010) found that the hot $\mathrm{O}$ dayside densities inferred from the Pioneer observations during high solar activity could only be reproduced on the basis of a forward scattering model but disregarding inelastic and quenching collisions. A similar result was obtained by Gröller et al. (2012) for the nightside, who calculated a nonthermal $\mathrm{O}$ density about an order of magnitude less than on the dayside.

Observations from missions preceding Venus Express have been summarized by Fox and Bougher (1991). Measurements with SPICAV could not confirm Pioneer Venus observations, although SPICAV should have been able to detect the hot O population around Venus if the number density was as indicated by the Pioneer Venus observations. Similarly, the ASPERA-4 instrument on board Venus Express could not measure a clear ENA signal from oxygen. According to recent model studies, the higher O ENA intensities derived by the pre-Venus Express studies were most likely based on too dense exospheric densities (Gröller et al. 2010). In addition, the density at low solar activity during the Venus Express era was up to a factor of 5 less dense than for the solar maximum conditions prevailing during Pioneer Venus

\subsection{Escape Processes}

Processes leading to escape of hydrogen and oxygen atoms may be grouped into four categories:

- thermal (or evaporative) escape;

- hydrodynamic escape;

- collisional nonthermal processes;

- solar wind induced escape.

A previous review of escape processes was given by Donahue et al. (1997) in relation to the evolution of water. A detailed discussion of the relative importance of these processes was given by Lammer et al. (2006) and modeling methods to evaluate the escape flux were described by Kallio et al. (2011). Atmospheric escape of hydrogen and oxygen from Venus is mainly influenced by the interaction of solar radiation and particle flux with the Venus unprotected planetary environment. Using a gas dynamic test particle model, they estimated the hydrogen pick rates and obtained an average loss rates for $\mathrm{H}^{+}$of about $1 \times 10^{25} \mathrm{~s}^{-1}$. They estimated the ion loss rates due to detached plasma clouds $\sim 0.5-1 \times 10^{25} \mathrm{~s}^{-1}$. Photochemically produced hot hydrogen atoms is a much more efficient loss mechanism for hydrogen with a global average total loss rate of $\sim 3.8 \times 10^{25} \mathrm{~s}^{-1}$. The $\mathrm{H}^{+}$ion pick up rate was estimated $1 \times 10^{25} \mathrm{~s}^{-1}$, much higher than the thermal escape rate of about $2.5 \times 10^{19} \mathrm{~s}^{-1}$. 
Thermal escape processes of oxygen atoms yield a negligible loss rate of about $10 \mathrm{~cm}^{-2} \mathrm{~s}^{-1}$ while hot atoms produced by $\mathrm{O}_{2}^{+}$recombination do not reach the escape energy for atomic oxygen of about $9 \mathrm{eV}$. Therefore, this particle population is gravitationally bounded to the planet but produces a hot oxygen corona. The loss of $\mathrm{O}^{+}$ions by solar wind pick up is about $1.6 \times 10^{25} \mathrm{~s}^{-1}$. Atmospheric sputtering by incident pick up $\mathrm{O}^{+}$ions give $\mathrm{O}$ atom loss rates in the order of about $6 \times 10^{24} \mathrm{~s}^{-1}$. Lammer et al.'s (2006) study concluded that the most efficient atmospheric escape processes for oxygen involve ions and are caused by the interaction with the solar wind. The results obtained indicate that the ratio between $\mathrm{H} / \mathrm{O}$ escape to space from the Venusian upper atmosphere is about 4, and closer to the stoichiometric $\mathrm{H} / \mathrm{O}$ escape ratio of 2:1. A detailed description of the escape rate caused by solar wind interaction with the Venus atmosphere is given by Futaana et al. (2017).

\section{The Ionosphere}

\subsection{The Venus Ionosphere in the Pre-VEX Era}

First observations of the electron density distribution in the Venus ionosphere by radio sounding as a measure of the total plasma density distribution were obtained during the flyby of Mariner 5 in 1967 (Mariner Stanford Group 1967; Kliore et al. 1967; Fjeldbo and Eshleman 1969). Many other US and soviet missions followed among these Mariner 10 (Howard et al. 1974; Fjeldbo et al. 1975), Venera-9 and -10 (Vasilev et al. 1980), Venera-15 and -16 and Magellan (Steffes et al. 1994; Jenkins et al. 1994). The largest volume of ionospheric radio sounding observations of the pre-VEX era, however, was collected by the Orbiter Radio Occultation (ORO) investigation on-board of Pioneer Venus Orbiter (PVO) from 1978 to 1989 (Kliore et al. 1979a, 1979b, 1991; Kliore and Luhmann 1991; Woo and Kliore 1991).

Constraints on the ionospheric composition and energetics came from PVO in-situ observations during low pericenter passes at $190 \mathrm{~km}$ altitude through the ionosphere from the Ion Mass Spectrometer OIMS (Taylor et al. 1979a, 1979b), the Retarding Potential Analyser ORPA (Knudsen et al. 1979a, 1979b), and the Electron Temperature Probe OETP (Brace et al. 1979). A summary of in-situ observations can be found in Brace et al. (1983). Brace and Kliore (1991) have written a thorough review and comparison of remote sensing and in-situ observations.

\subsection{Formation and General Structure of the Venus Ionosphere}

Figure 28 shows exemplarily the general structure of a quiet Venus ionospheric electron density distribution. The two-layer structure of the lower ionosphere with the lower "V1"1 layer at an radius of $6180 \mathrm{~km}^{2}$ (altitude of $130 \mathrm{~km}$ ) and the main "V2" layer at a radius of $6190 \mathrm{~km}$ (altitude $140 \mathrm{~km}$ ) is prominent (Pätzold et al. 2007; Girazian et al. 2015). This specific electron density profile was observed on DOY017, 2008, by the Venus Express

\footnotetext{
${ }^{1}$ Other researchers call the lower and the main layer E and F1 layer, respectively, in order to compare with similar formations in the Earth ionosphere (e.g. Bauer et al. 1977; Banks and Kockarts 2013; Fox 2007). We adopt the notation of Rishbeth and Mendillo (2004) for the Mars ionosphere and call the layers V1 and V2 where "V" stands for Venus. This avoids confusion when comparing planetary ionospheres.

${ }^{2}$ The processing of radio occultation data yields the refractivity as a function of radius with respect to the center of planet. The altitude assignments are principally relative to the Venus geoid which is realized by the mean planetary radius of $6050.8 \mathrm{~km}$ because of the non-oblate, spherical shape of the planetary body.
} 
Fig. 28 Quiet electron density profile of the Venus ionosphere derived from VEX-VeRa radio occultation on DOY 017, 2008, during egress at $\mathrm{X}$-band (open circles) and differential Doppler (filled circles). The electron density distribution is called "quiet" because both profiles derived from the X-band and the differential Doppler agree very well and the distribution is not perturbed by other effects. Panel (a): full profile from $6150 \mathrm{~km}$ radius (about $100 \mathrm{~km}$ altitude) to $6450 \mathrm{~km}$ radius $(400 \mathrm{~km}$ altitude). The mean noise level of the differential Doppler profile is at about $2,300 \times 10^{6} \mathrm{~m}^{-3}$. The ionopause (a strong gradient towards the noise level) is at $6300 \mathrm{~km}$ radius; panel (b): is a zoom of (a): the lower ionosphere is dominated by the main layer V2 and the lower layer V1 at $6190 \mathrm{~km}(140 \mathrm{~km}$ altitude) and $6180 \mathrm{~km}(130 \mathrm{~km}$ altitude), respectively. The base of the ionosphere is at about $6160 \mathrm{~km}$ (110 km altitude). The left dash-dotted vertical lines are the 1- $\sigma$ noise level, the right dash-dotted lines are the 3- $\sigma$ noise level. Figure courtesy VEX-VeRa team
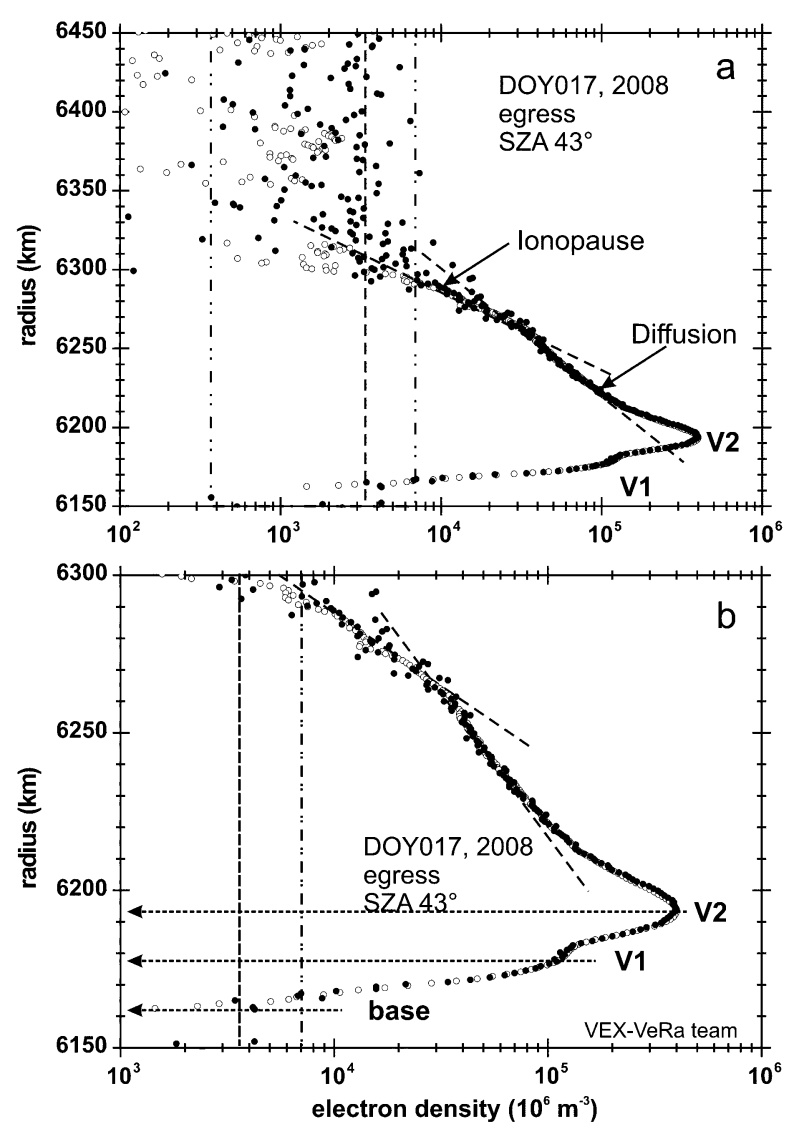

Radio Science Experiment VeRa (Häusler et al. 2006) by radio occultation at X-band and at simultaneous S-band frequencies (differential Doppler).

Solar radiation at EUV (15-100 nm) and soft X-ray $(1-15 \mathrm{~nm})$ ionize the $\mathrm{CO}_{2}$ molecule which is the main species of the neutral atmosphere up to $200 \mathrm{~km}$ altitude. Rapid molecular reactions with neutral atomic oxygen form $\mathrm{O}_{2}^{+}$via (Schunk and Nagy 1980)

$$
\mathrm{CO}_{2}^{+}+\mathrm{O}_{2} \rightarrow \mathrm{O}_{2}^{+}+\mathrm{CO}
$$

The $\mathrm{O}_{2}^{+}$ion is the dominant ion in the ionosphere up to $200 \mathrm{~km}$, the original ion $\mathrm{CO}_{2}^{+}$is a minor species of $1 \%$ to $10 \%$ by volume.

Another ion produced by chemical reactions (7.1) is $\mathrm{O}^{+}$which is also transformed to $\mathrm{O}_{2}^{+}$ by chemical reactions with $\mathrm{CO}_{2}$ below $200 \mathrm{~km}$ (see (7.1)) but is produced above $200 \mathrm{~km}$ by photoionization and becoming there the dominant ion.

The general layered structure of the lower ionosphere below $200 \mathrm{~km}$ is maintained by the photo-chemical equilibrium balance of production and loss. In this photo-chemical equilibrium region the $\mathrm{O}_{2}^{+}$ions and electrons are lost by dissociative recombination (see (7.1)).

The V2 layer is formed primarily by photo-ionization in the EUV spectral range and by only a small fraction by impact ionization of energetic electrons. The contribution from secondary ionization is in the order of $10 \%$ as shown by Peter et al. (2014) by comparisons between model calculations and VeRa electron density measurements. 
The primary photo-ionization by X-rays cannot account for the observed V1 peak electron densities. The secondary impact ionization by energetic electrons is dominant (Peter et al. 2014).

\subsection{VEX-VeRa Observations, Detailed Structures and Layers}

The full structure of the ionosphere can only be assessed by remote sensing radio occultation methods. This reveals the electron density distribution as a function of radius with respect to the center of the planet or altitude when applying the mean planetary Venus radius of $6050.8 \mathrm{~km}$. As a matter of fact, however, the electron density distribution stands for the total ion or plasma distribution. The composition can be assessed in-situ during low (<190 km) pericenter passes which was done primarily by Pioneer Venus Orbiter (PVO) (Bauer et al. 1977; Brace et al. 1983; Bougher et al. 1989). Figure 29 shows a number of ionospheric electron density profiles observed by VeRa. VeRa used two coherent one-way downlink frequencies at X-band $(8.4 \mathrm{GHz})$ and S-band $(2.3 \mathrm{GHz})$ stabilized by an Ultrastable Oscillator (USO) (Häusler et al. 2006) for the radio sounding of the neutral atmosphere (Tellmann et al. 2009, 2012) and the ionosphere (Pätzold et al. 2007, 2009; Peter et al. 2014). Figure 29 also shows the great advantage by using two coherent downlink frequencies (differential Doppler). The latter allows the derivation of the true plasma distribution in the ionosphere in particular when compared to the profile derived from a single radio frequency (at $\mathrm{X}$ band). Certain variations in the electron density profile derived from the X-band are caused by small-scale spacecraft bus variations or other vibrational disturbances at the ground station which produce small-scale Doppler velocity contributions along the line-of-sight which are translated into electron density. These Doppler velocity contributions are compensated by using two frequencies.

Figure 29a shows the full profile from the base at $6150 \mathrm{~km}(100 \mathrm{~km}$ altitude) to the ionopause. The ionopause feature itself is a strong gradient of electron density over a short altitude range (30-50 km). The absolute altitude may vary strongly (Fig. 29) between $6300 \mathrm{~km}$ to and about $6450 \mathrm{~km}$ radius (250 km to $400 \mathrm{~km}$ altitude towards the noise level). at about $6350 \mathrm{~km}$ radius (300 km altitude towards the noise level. The ionopause altitude varies depending on the kinetic state of the solar wind and the solar activity (Fig. 29). The electron density noise level of the profile in Fig. 28 is about 2,300 $\times 10^{6} \mathrm{~m}^{-3}$ at differential Doppler in Fig. 28a, somewhat higher than the noise level in the profile derived from single $\mathrm{X}$-band frequency. The electron density noise level strongly depends on the turbulence of the solar wind (the radio signal must propagate through interplanetary space before being received at the ground station on Earth) and the elongation angle of Venus relative to the solar disk as seen from the Earth. The electron density noise increases with decreasing elongation angle. Therefore, the observed electron density noise is not necessarily a property of the ionosphere and does not describe the inherent ionospheric turbulence but is dominated by the radio propagation through the turbulent solar wind outside of the ionosphere. A noise level of $1,000 \times 10^{6} \mathrm{~m}^{-3}$ is a typical mean value for the entire VEX mission. One may encounter a few $100 \times 10^{6} \mathrm{~m}^{-3}$ during inferior conjunction at X-band or extremely high levels during solar conjunction which are then a description of the plasma turbulence within the solar corona. The electron density $n_{e}$ decreases between the topside above typically $6250 \mathrm{~km}$ radius ( $200 \mathrm{~km}$ altitude) to the ionopause altitude exponentially

$$
n_{2}=n_{0} \exp \left\{-\frac{z-z_{0}}{H_{p}}\right\}
$$




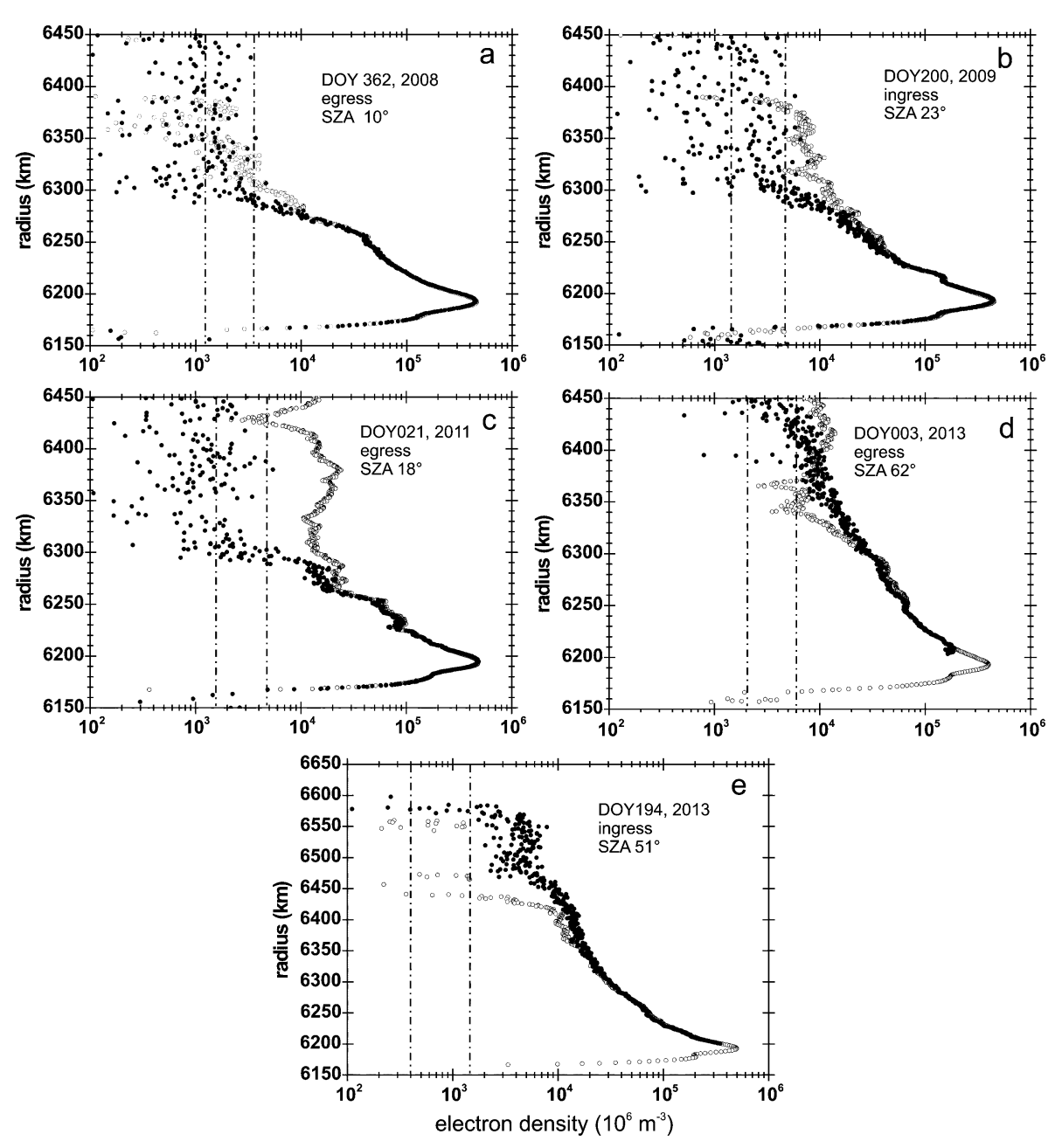

Fig. 29 VeRa electron density profiles showing the variability over the mission time. Profiles were derived from single frequency at X-band (open circles) and dual-frequency differential Doppler (filled circles). The true plasma distribution is displayed by the differential Doppler profile. The deviations of the X-band profile are caused by small-scale spacecraft bus vibrations or other vibrational disturbances at the ground station which produce an extra Doppler contribution along the line-of-sight and perturb the derived electron density profile. The left dash-dotted vertical line in all panels is the 1- $\sigma$ noise level, the right dash-dotted line is the 3- $\sigma$ noise level. Figure courtesy VEX-VeRa team

where $H_{p}$ is the plasma scale height and $n_{0}$ is the electron density at altitude $z_{0}$. The plasma scale height and therefore the plasma temperature defines the slope of the decrease in density and may vary over wide ranges from occultation to occultation. Values between $20 \mathrm{~km}<H_{p}<120 \mathrm{~km}$ have been observed during the VEX mission. Many electron density profiles show a bulge in the topside (Pätzold et al. 2007) between $6200 \mathrm{~km}(150 \mathrm{~km}$ altitude) and $6250 \mathrm{~km}(200 \mathrm{~km})$ (Fig. 29). Simulations and models have shown that this bulge is not formed by photo-ionization (Peter et al. 2014). The bulge is also not present in ionospheric standard models (e.g. in Fox 2007, although this model is constrained to very high 


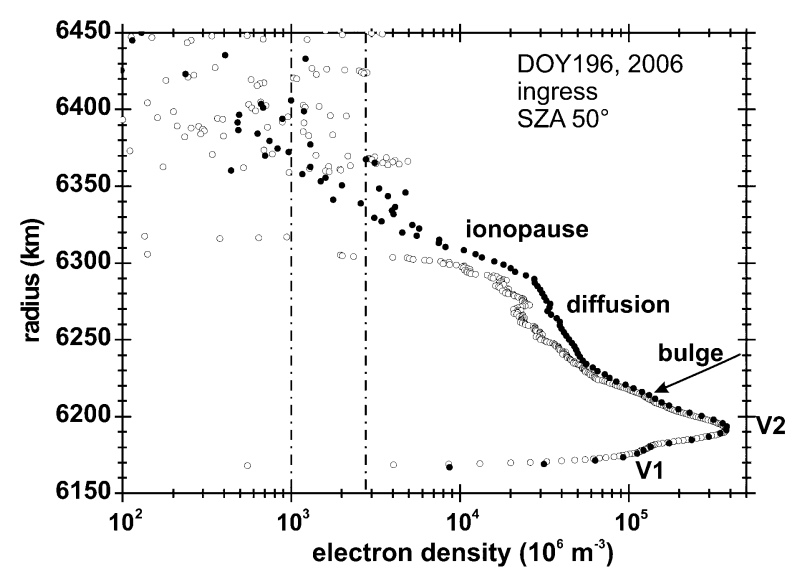

Fig. 30 VeRa electron density profile from DOY 196, 2006, derived from single frequency X-band (open circles) and simultaneous dual-frequency recordings (closed circles) which represent the true plasma distribution. The V1 and V2 layers are well resolved, the diffusion region starts at $6235 \mathrm{~km}$ radius (185 $\mathrm{km}$ altitude), followed by the ionopause at $6290 \mathrm{~km}(240 \mathrm{~km}$ altitude). There is a "bulge" between the V2 peak altitude at $6195 \mathrm{~km}$ (145 km altitude) and the diffusion region (arrow) which is not formed by photoionization as emphasized by a comparison with a Chapman function (solid line) for V2. Figure redrawn from Pätzold et al. (2007)

solar zenith angles). There are also no reports from earlier radio sounding experiments, in particular PVO. There is currently no definite solution on the formation of the bulge.

The two prominent features of the electron density distribution are the two layers V1 and V2 (Figs. 28 and 30). The lower V1 layer is a common feature in the Venus ionosphere, formed by solar X-ray and secondary ionization, showing its shape more or less pronounced as a function of solar zenith angle (SZA) and solar activity (Girazian et al. 2015). The V1 layer was seldom described in past missions. Breus et al. (1985) reported its existence from Venera-15 occultations; Kliore et al. (1979b) reported only once about the V1 layer for high solar zenith angles; Cravens et al. (1981) called it a "ledge" below the V2 layer. Comparing VeRa electron density profiles from solar minimum and solar maximum, the V1 layer is in particular strong and pronounced at solar maximum (Figs. 29 and 31) when the solar X-ray flux is higher than at solar minimum.

The V1 layer appears in models based on photo-ionization and secondary ionization (Fox and Sung 2001; Fox 2007; Peter et al. 2014). Although these models were all based on inadequate models of the background neutral atmosphere at ionospheric altitudes (the Hedin model for the models by Fox (2007); VENUSGram and VIRA for the model by Peter et al. (2014)), it is clear that secondary ionization by impacts of energetic electrons formed by solar soft X-rays is the dominating ionization process up to ten times stronger than the primary ionization by solar photons.

Distinct, low-lying layers of electron density below the base of the ionosphere were detected by VeRa (Pätzold et al. 2009). The layers appear sporadically and occasionally. They could be identified on the dayside only and appear to be geographically localized as they usually occur either on the northern or southern hemisphere of the same VEX orbit. The low electron density layers are detected at all latitudes but only for $55^{\circ} \leq \mathrm{SZA} \leq 90^{\circ}$ (Pätzold et al. 2009). Withers et al. (2013) found some evidence for the appearance of lower layers in electron density profiles from earlier Venus missions as Mariner-10, Venera-9 and -10 and Pioneer Venus Orbiter. 


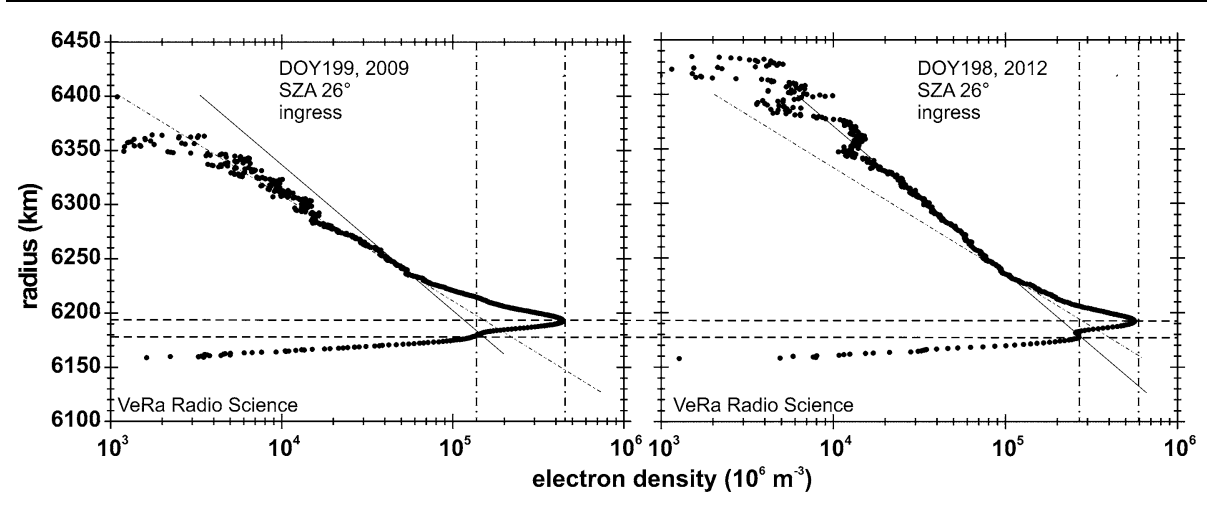

Fig. 31 Two VeRa electron density profiles observed at solar minimum (panel a) and solar maximum (panel b). Both layers V1 and V2 appear stronger and more pronounced at solar maximum than at solar minimum (dashed-dotted vertical lines). There is no obvious change in the altitudes of the V1 and V2 layers (dashed horizontal lines). The slope of the diffusion region is also steeper at solar maximum (solid line) then at solar minimum (dash-dotted inclined line). Figure courtesy VEX VeRa team

Figure 32 shows three examples of extra electron density layers (Pätzold et al. 2009): panel (a) a fully detached layer well below the base of V1, panel (b) a layer merged with the base of V1 and panel (c) two distinct layers, one partially merged, the other one well detached and at very low altitudes.

In fact, sporadic plasma layers were expected and predicted (Pesnell and Grebowsky 2000; Grebowsky et al. 2002; Pesnell et al. 2004; Molina-Cuberos et al. 2008) and believed to be caused by the surface ablation of metallic atoms from incident meteors and subsequent ionization. Such a "meteor layer" contains metallic ions, namely iron and magnesium. A well-known phenomenon in the Earth ionosphere, it was also predicted for the Mars ionosphere (Pesnell and Grebowsky 2000; Molina-Cuberos et al. 2008) and discovered by the MaRS radio science team on Mars Express (Pätzold et al. 2005; Withers et al. 2013).

The VeRa data (Pätzold et al. 2009) suggest that the "meteor layer" phenomenon is sporadic and not a permanent dayside feature with regard to the sensitivity of the radio science method. The existence of a night-side "meteor layer" is difficult to identify because of the unstructured night-time ionization (Pätzold et al. 2007).

There is convincing evidence that the lower layers below V1 are formed by incident meteors. There are, however, results from modeling which show a $\mathrm{NO}^{+}$layer or ledge below V1 at altitudes where meteor layers are observed (Fox and Sung 2001). This "NO $\mathrm{NO}^{+}$layer" is formed by quite a number of chemical reactions between the basic neutral and ionized species of the atmosphere and ionosphere. Such a lower layer should therefore be a standard feature of the ionosphere. The potential ion densities (or equivalent electron densities) are above typical electron density noise levels of radio occultation experiments in particular at solar maximum. There is, however, no strong observational evidence for such a lower layer in the "everyday-day" ionosphere.

\subsection{Variability of the Venus Ionosphere}

\subsubsection{Solar Flux and Solar Activity}

Both, the V1 and the main layer V2 react strongly to changes in solar flux in the X-ray and EUV wavelength ranges, respectively (Kliore and Luhmann 1991). Changes in solar flux are 

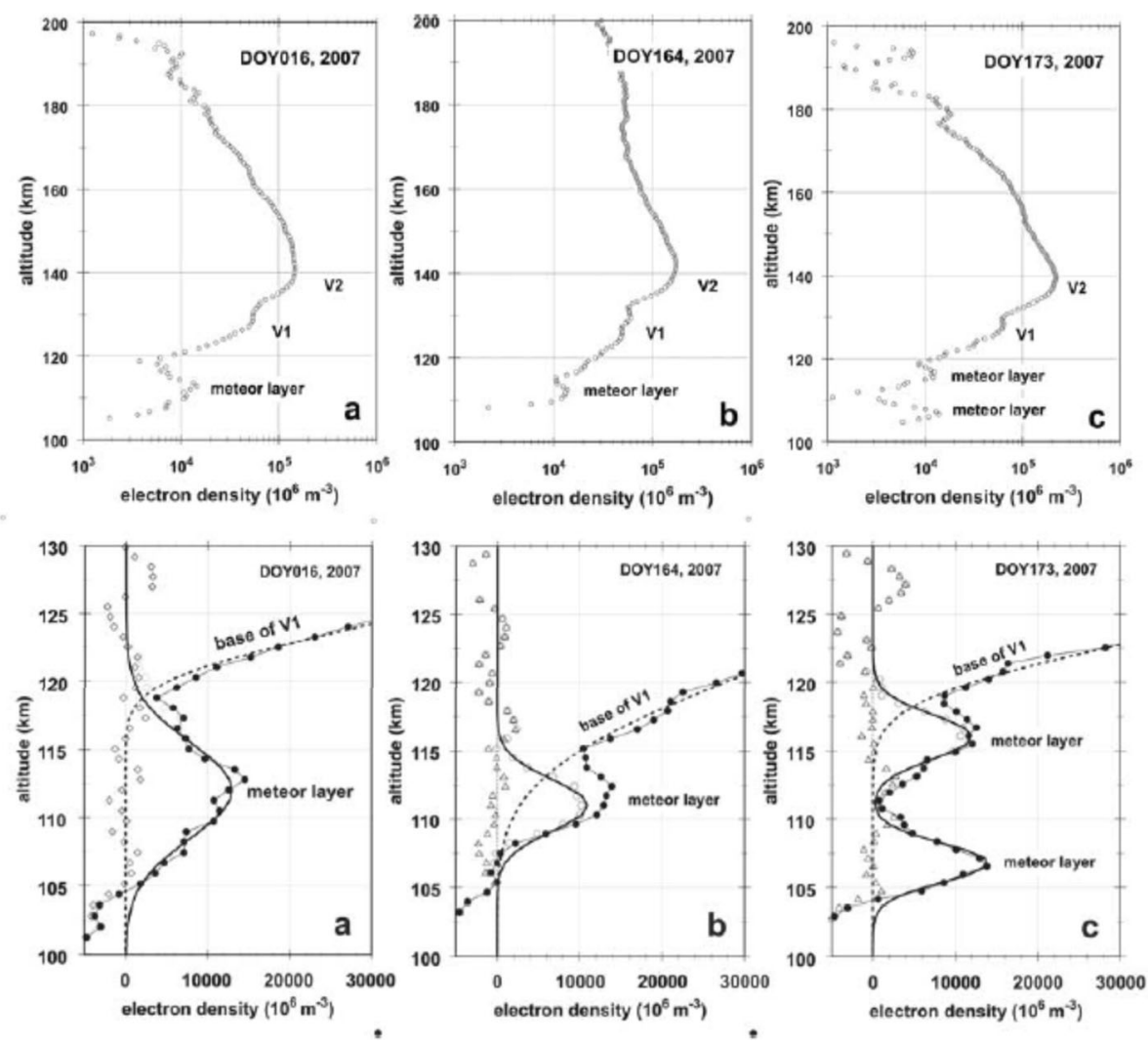

Fig. 32 Three examples for "meteor layers" below $120 \mathrm{~km}$ (6170 km radius), the altitude of the lower layer $\mathrm{V} 1$, in the Venus ionosphere as observed by VeRa. The upper row shows the full electron density profiles; lower row shows the altitude range between $100 \mathrm{~km}$ and $130 \mathrm{~km}$ altitude $(6250 \mathrm{~km}$ to $6180 \mathrm{~km}$ radius). Filled circles are the observed electron density profile; open circles are the difference between the observation and a fit on the base of V1 (dashed line) and are supposed to represent the "meteor layer" density distribution; the solid line is a Gauss fit on the open circles and the open triangles are the residual differences between the open circles and the Gauss fit. Panel (a): meteor layer detached from the V1 layer; panel (b): meteor layer merged with the base of the V1 layer; panel (c): double meteor layer, one detached and one merged with V1. Figure taken from Pätzold et al. (2009)

caused by solar flaring (strong at X-ray), solar rotation and solar cycle (both wavelengths but stronger at EUV). An orbital effect is not present with Venus because of the near-circular orbit in contrast to Mars (Peter et al. 2014).

Figure 33 shows the integrated solar flux at the position of Venus over the VEX mission time (taken from Peter et al. 2014). The pronounced deep solar minimum from 2007 to the end of 2009 is clearly visible. There is a change in flux between solar minimum and maximum by $25 \%$. Short-period peaks during the declining and raising phase of solar cycles 23 and 24, respectively, are caused by the solar rotation. Note that the flux during solar minimum was nearly constant without pronounced peaks. Figure 31 compares two electron density profiles from solar minimum (DOY 199, 2009) and solar maximum (DOY 198, 2012) for the same SZA. The V1 and the V2 peaks are more pronounced at solar maximum than at solar minimum. The higher V2 peak density at solar maximum is caused by the higher solar flux. 


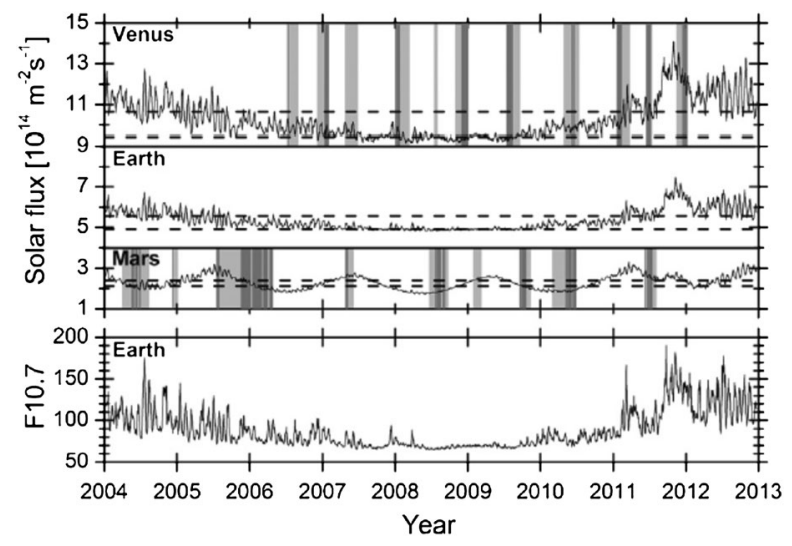

Fig. 33 Integrated solar flux between $0.1 \mathrm{~nm}$ and $95 \mathrm{~nm}$ (X-ray to EUV) at the position of Earth (Tobiska et al. 2000) and calculated for the positions of Venus and Mars (from 2004) solar cycle 23 to 2012 (shortly after the start of solar cycle 24). Spikes are flux changes caused by the solar rotation; the large-scale variation at Mars is caused by the elliptical Mars orbit. Clearly seen is the deep solar minimum from 2007 to the end of 2009 and the rise of activity at the start of the new solar cycle in 2010. The light and dark grey bars are the occultation seasons. The dark bars are nighttime observations. The lower panel shows the F10.7 solar radio flux as a proxy for solar activity (from Peter et al. 2014)

Short-term changes of solar flux at the position of Venus like solar flaring (few hours duration) and even changes caused by solar rotation are then contained in the scatter of the peak densities for a given solar cycle phase (Fig. 34a).

\subsubsection{Solar Zenith Angle}

Figure 34 is the typical "Chapman Plot" of V2 peak electron density and V2 peak altitude as a function of Solar Zenith Angle (SZA). Chapman theory predicts for a one-species atmosphere at constant temperature ionized by a single wavelength radiation that the peak density $n_{e}$ varies as

$$
n_{e}(S Z A)=n_{e, 0}(S Z A=0) \cdot \cos ^{0.5}(S Z A)
$$

and the peak altitude $h$ as

$$
h(S Z A)=h_{0}(S Z A=0)-H \cdot \ln (\cos (S Z A))
$$

where $H$ is the neutral scale height.

Figure 34a shows the V2 peak density observed by VeRa as a function of SZA. The branching of the peak density values for small SZA depending on the solar flux is clearly resolved. Both the solar minimum and maximum peak density distributions in Fig. 34a follow well the ideal Chapman relation (7.3). Comparing Fig. 33a with the similar Figure in Cravens et al. (1981) (Fig. 35) reveals that the VeRa solar minimum V2 peak density follows exactly the Venera solar minimum densities. The VeRa solar maximum V2 peak densities are well below the PVO V2 peak densities. This is easily explained by the strength of the solar cycles 21 with its maximum in 1980 and cycle 24 with its maximum in 2014. Cycle 21 appears much stronger than cycle 24 (Fig. 36). The V2 peak density is therefore clearly under solar control. 
Fig. 34 Panel (a): V2 peak density as a function of solar zenith angle as observed by VeRa from 2007 to 2014. Filled circles are data from solar minimum (2007 to 2010) and open circles are data from solar maximum (2010 to 2014). The solid and dashed curves are ideal Chapman relations according to Eq. (7.3). The vertical dashed line marks the terminator. Panel (b): V2 peak altitude as observed by VeRa. The peak altitude remains constant at $140.7 \mathrm{~km} \pm 2.1 \mathrm{~km}$ $(3 \sigma)$ for $\mathrm{SZA} \leq 80^{\circ}$ (middle dashed dotted line). Although the scatter increases for increasing SZA, the altitude remains constant on average. The expected increase is seen for $\mathrm{SZA} \geq 80^{\circ}$ and decreasing for $90^{\circ} \leq \mathrm{SZA} \leq 100^{\circ}$. Altitudes for $\mathrm{SZA} \geq 100^{\circ}$ are uncorrelated according to a random nighttime structure. The upper and lower dashed dotted horizontal lines indicate the $3 \sigma$ noise level.

Figure courtesy VEX VeRa team

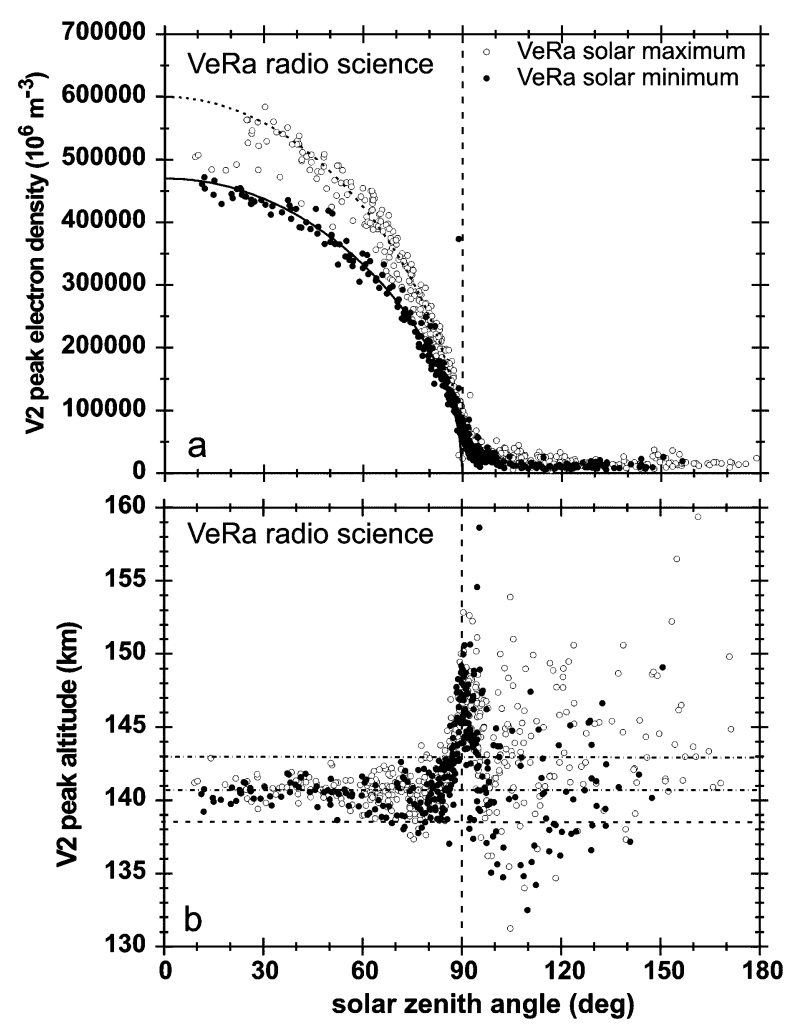

Figure 34b shows the V2 peak altitude observed by VeRa as a function of SZA. The peak altitude is constant at $140.7 \pm 0.75 \mathrm{~km}$ for $10^{\circ}<\mathrm{SZA}<60^{\circ}$ as already reported from PVO data (Cravens et al. 1981). Cravens et al. (1981), however, observed a decrease in altitude for $60^{\circ}<\mathrm{SZA} \leq 85^{\circ}$ while the peak altitude increased strongly again near the terminator. The drop in altitude in the PVO V2 altitudes was explained as the cooling of the atmosphere for large SZA. The VeRa observations are in contrast to the PVO observations by Cravens et al. (1981). VeRa observed a non-varying thermal structure towards the terminator (Fig. 34). VeRa data fill the range of $140.7 \pm 2.1(3 \sigma) \mathrm{km}$ uniformly in contrast to the PVO data for $60^{\circ}<\mathrm{SZA}<85^{\circ}$. The discrepancy might at least partially be explained by the statistical difference in the PVO and VeRa data sets. The number of VeRa occultations on the dayside is more than three times larger than those from PVO. There is also no identifiable solar cycle effect in the VeRa altitude data towards the terminator. Solar maximum and solar minimum peak altitudes are evenly distributed over the entire range of SZA.

Girazian et al. (2015) studied the behavior of the V1 layer based on 200 VeRa electron density profiles. The V1 layer was characterized with respect to peak density, peak altitude and morphology as a response to changes in the SZA and solar activity. The V1 peak altitude does not change as much as the V2 altitude that is also explained as a constant thermal structure of the neutral atmosphere at ionospheric altitudes towards the terminator. The solar X-ray flux changes stronger than EUV with increasing solar activity and the V1 shape appears more pronounced at solar maximum than $\mathrm{V} 2$. 
Fig. 35 V2 peak electron density as a function of SZA (taken from Cravens et al. 1981). Venera 9 and 10 peak densities from solar minimum (open circles) and PVO peak densities from solar maximum 1980 (stars, filled points, open circles with points) follow the Chapman relation (7.3) shown as dashed curves. The VeRa solar minimum peak densities from Fig. 34 follow the Venera solar minimum densities; the VeRa solar maximum peak densities from Fig. 34 are in between the Venera and $\mathrm{PVO}$ peak densities in this Figure

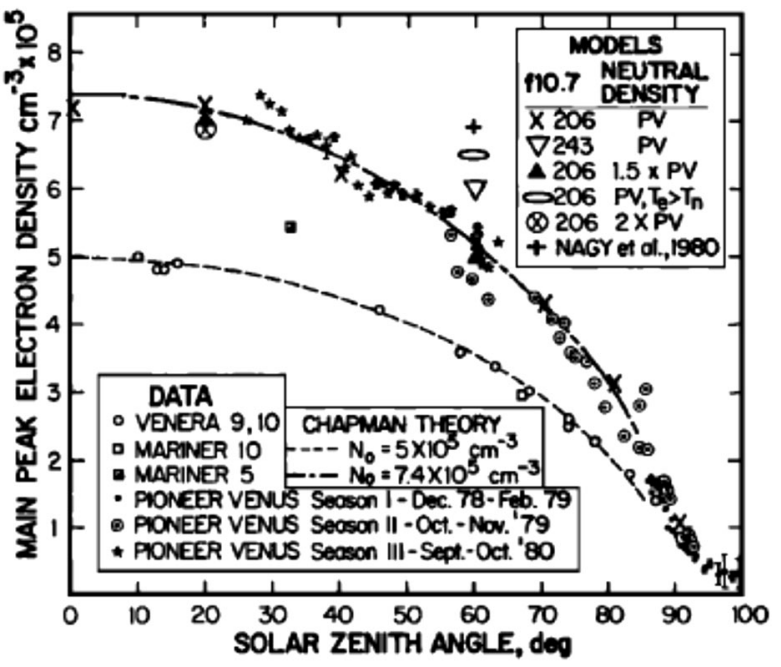

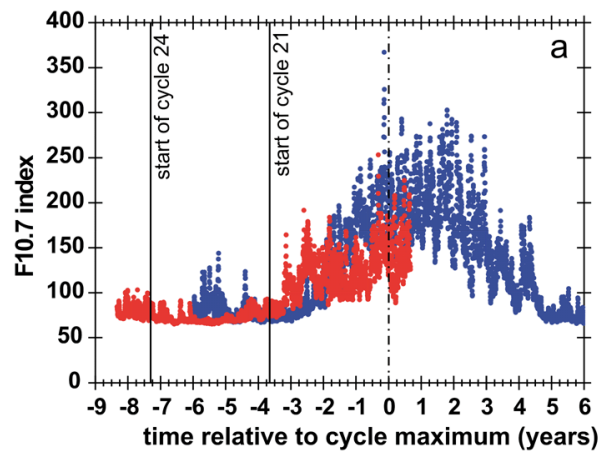

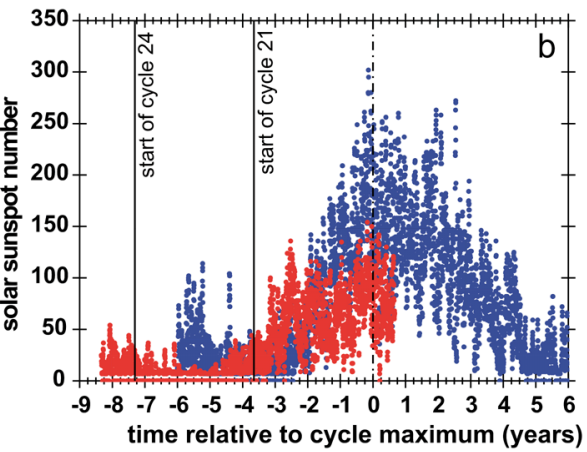

Fig. 36 Panel (a): solar F10.7 index for solar cycle 21 (blue dots) and solar cycle 24 (red dots) relative to the time of the respective solar maximum (dash-dotted line). Note that the maximum of solar cycle 24 is about half the strength of the maximum of cycle 21. Note also the pronounced weak solar minimum of cycle 24 . The starts of cycle 21 and 24 are indicated by the solid vertical line. Panel (b): same as panel (a) but as solar sunspot number. The data points in both panels are daily averaged values

\subsubsection{Ionopause Altitude}

The ionopause is the boundary between the kinetic pressure of the solar wind hitting the upper atmosphere and the plasma pressure of the ionosphere. The ionopause altitude at Venus was defined the PVO in-situ instruments as that altitude where the plasma density drops below $100 \times 10^{6} \mathrm{~m}^{-3}$ at a steep gradient (Brace et al. 1983). The Venus express magnetometer MAG observed a drop in magnetic field strength at that ionopause altitude (Zhang et al. 2008). The noise level of VeRa (but also PVO ORO) radio occultation electron density profiles is much higher, $1,000 \times 10^{6} \mathrm{~m}^{-3}$ on average depending on the turbulent state of the solar wind. Nevertheless, a steep electron density gradient is mostly observed on top of the diffusion region. Kliore and Luhmann (1991) defined the ionopause altitude for PVO ORO radio occultation electron density profiles when the electron density falls below $500 \times 10^{6} \mathrm{~m}^{-3}$ regardless whether there is a steep or gradual (even directly from the diffusion region) drop of electron density (Fig. 30). If the electron density noise level is high 
Fig. 37 Venus ionopause altitudes as a function of SZA as observed by VeRa from 2007 to 2014. There is a clear separation of the ionopause altitudes for solar minimum or maximum conditions. Figure courtesy VEX VeRa team

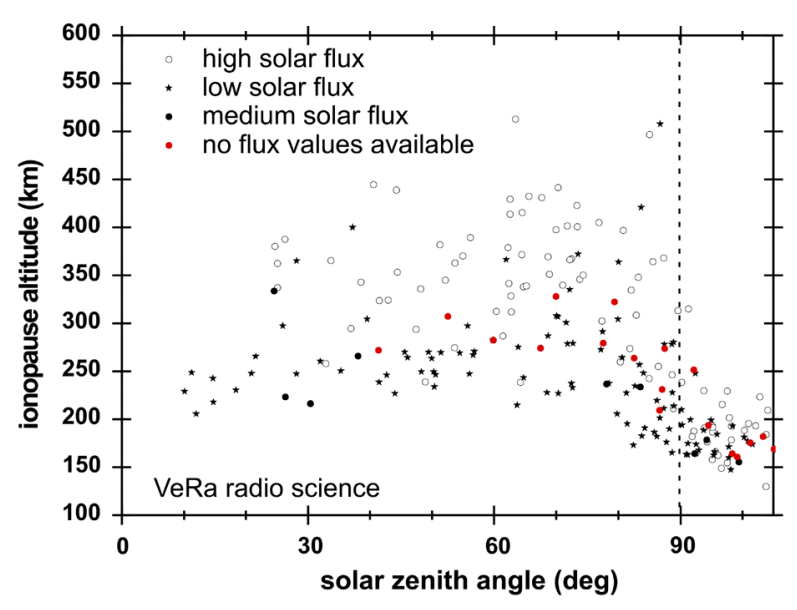

and the density never drops below $500 \times 10^{6} \mathrm{~m}^{-3}$, the altitude was chosen where the profile merged with the noise level.

VeRa profiles derived from differential Doppler rarely showed a noise level less than $1,000 \times 10^{6} \mathrm{~m}^{-3}$ but show mostly a strong gradient starting from the top of the diffusion region towards the noise level over a few $10 \mathrm{~km}$ altitude range (Figs. 28, 29 and 30). Following the definition by Kliore and Luhmann (1991), Fig. 37 shows the ionopause altitudes for low, medium and high solar flux as a function of SZA. Solar minimum ionopause altitudes are fairly constant between $200 \mathrm{~km}$ and $275 \mathrm{~km}$ over the entire range of SZA. Solar maximum ionopause altitudes are mostly higher than $275 \mathrm{~km}$, indicating a reduced pressure from the solar wind and/or increased ionospheric plasma pressure from increased ionization by the solar maximum flux.

Both solar minimum and maximum ionopause altitudes drop below $200 \mathrm{~km}$ and $275 \mathrm{~km}$, respectively, for $\mathrm{SZA}>80^{\circ}$. This corresponds mostly with PVO observations although the drop for SZA $>80^{\circ}$ was not observed (Kliore 1992).

\section{Summary and Perspectives}

In this section, we summarize some of the major advances in aeronomy of the Venus upper atmosphere during the last 20 years and address some of the remaining questions and perspectives.

The SPICAV and VIRTIS remote sensing instruments on board Venus Express have provided a wealth of new results and have significantly improved knowledge of Venus' upper atmosphere aeronomy. Multispectral observations of day and night side airglow have proven to be a powerful tool in the fields of thermal structure, chemical composition, ionospheric structure, transport and neutral escape.

Analysis of VIRTIS-H high-resolution spectra of the dayside non-thermal emission of $\mathrm{CO}$ has provided temperature determinations in the 100 to $150 \mathrm{~km}$ range that complement other daytime measurements and SPICAV-UV nightside temperature profiles derived from stellar occultations.

Ground-based spectroscopy of the $\mathrm{O}_{2}$ band at 1.27 micron has provided reliable and accurate temperature determination of the nightside temperature at the altitude of the emission layer. VIRTIS-M limb images have shown that the peak altitude of this strong nightglow emission is located between 97 and $100 \mathrm{~km}$, putting a strong constraint on models of 
the thermal structure and less accurate measurements. An exhaustive compilation of dayside and nightside temperature measurements based on different observational approaches is given by Limaye et al. (2017). This determination of the altitude of the airglow layer now makes it possible to assign the temperature derived from ground-based spectroscopy to a narrow range of altitudes in the Venus mesosphere.

The neutral composition in the mesosphere-thermosphere transition region was largely unexplored by previous space missions. Significant progress has been made in the recent years both by Venus Express remote sensing instruments and ground-based spectral observations. Combination of UVIS-Cassini spectral observations during the Venus Cassini flyby with the SPICAV-UV and VIRTIS-M and ground-based telescopes have generally confirmed the validity of empirical and three-dimensional models that were mostly based on measurements collected during the Venus Express mission. The atomic oxygen density distribution is now fairly well determined based on analysis of the $\mathrm{O}_{2} 1.27 \mu \mathrm{m}$ emission on the night side and the ultraviolet airglow on the dayside. The detection of ozone near $100 \mathrm{~km}$ with SPICAV-UV has prompted an upward revision of the amount of chlorine in the nighttime transition region. It confirms the role played by ozone in the excitation of the Meinel bands in the hydroxyl nightglow through the $\mathrm{O}_{3}+\mathrm{H}$ reaction. The similarity between the morphology of the vibrationally excited $\mathrm{OH}$ and the $\mathrm{O}_{2}{ }^{1} \Delta$ nightglows observed with VIRTIS strongly argues in favor of $\mathrm{O}$ atoms as a common precursor to both emissions.

By contrast, statistical and simultaneous mapping of the $\mathrm{O}_{2}{ }^{1} \Delta$ and the NO UV emissions exhibit clear spatial morphological differences. This lack of spatial correlation clearly indicates that these two nightglow layers, although vertically separated by less than $20 \mathrm{~km}$, have a different response to the varying dynamical and photochemical conditions. Unfortunately, simultaneous global imaging of the two airglow emissions is still missing and a full understanding of the relative contribution of the subsolar to antisolar circulation and the zonal transport is missing. Similarly, $\mathrm{CO}$ molecules are carried by the subsolar to antisolar global circulation. Analysis of the $\mathrm{CO} 4.7 \mu \mathrm{m}$ dayside emission observed with VIRTIS-H argues for a $\mathrm{CO}$ distribution controlled by dynamics in the lower thermosphere, with dominant subsolar to antisolar transport similar to the $\mathrm{O}$ and $\mathrm{N}$ atoms. Imaging of the $\mathrm{O}_{2} \mathrm{IR}$ nightglow and measurements of UV airglow have revealed a high level of variability in the morphology and brightness within period of days or hours. The sources of this variability are still largely unknown and attempts to include them in 3-D models have not provided conclusive results so far.

Ground-based measurements of auroral green line emission in relation with solar events such as coronal mass ejections confirm that Venus is also responsive to solar activity. Other auroral emissions (OI $130.4 \mathrm{~nm}$, CO Cameron bands) were also occasionally observed with the PV-OUVS spectrometer, but the relations with solar activity could not be directly demonstrated.

Extensive remote sensing coverage of the electron density in the Venus ionosphere has been performed during the full Venus Express mission. The VeRa measurements confirmed the presence of a "meteor layer" phenomenon, which is a sporadic and not a permanent dayside feature. The altitude of the ionopause was shown to vary with solar activity.

The distribution of thermal and hot hydrogen atoms has been determined from Lyman- $\alpha$ limb scans. They confirmed the presence of a dominant population of nonthermal $\mathrm{H}$ atoms at high altitude and showed that the transition between the hot and cold populations depends on local time.

A series of questions remain unanswered. Others have been raised on the basis of Venus Express and recent ground-based observations and model developments. 
- What are the sources of the variability observed in the airglow emissions on the nightside? The $\mathrm{NO}, \mathrm{O}_{2}$ and $\mathrm{OH}$ emissions all involve recombination of species created on the dayside and transported on the nightside by the global circulation where they chemically recombine and generate airglow photons. Observations indicate variations in solar flux only play a minor role in the rapid changes observed in the nightglow morphological distribution. These time variations and the separation observed between regions of enhanced nitric oxide and oxygen nightglow suggest a non-steady transport. Gravity waves have been suggested as a source of variability but have not been demonstrated to provide the required amplitude. A future mission combining global viewing of several emissions involving different constituents would help to clarify this issue.

- The importance of orography on the analysis of the airglow brightness and morphology is still unclear in spite of efforts to account for changes in optical thickness related to the airglow altitude relative to the surface.

- The difference observed in global and local morphology of the $\mathrm{NO}$ and $\mathrm{O}_{2}$ emissions was largely unexpected. It probably stems from a different statistical wind regime prevailing between 97 and $115 \mathrm{~km}$. In particular, the observed shift of the NO nightglow peak intensity toward the dawn terminator compared to the statistical near midnight $\mathrm{O}_{2}$ statistical maximum is still unexplained. It may imply that the zonal wind component decreases above the cloud top to become quite small near $95 \mathrm{~km}$, but picks up speed at higher altitude. Simultaneous global imaging of the two nightside emissions in a future space mission would provide key clues to understand the changing wind pattern in the mesosphere-thermosphere region.

- Although analysis of the limb CO emission near 4.7 microns has yielded information for dayside temperatures and $\mathrm{CO}$ vertical distributions, the $\mathrm{CO}_{2}$ emissions at 4.3 microns have as yet only been analyzed in the nadir geometry. The large dataset of VIRTIS limb observations of the $\mathrm{CO}_{2}$ band has not yet been exploited to determine temperature profiles, $\mathrm{CO}_{2}$ densities and variability. The large optical thickness in this geometry, relevant for many $\mathrm{CO}_{2} 4.3 \mu \mathrm{m}$ bands up in the thermosphere, considerably complicates model calculations. Current ongoing projects in the analysis of similar emissions from Mars Express (Piccialli et al. 2016; http://www.upwards-mars.eu/) should lead to a more operational tool to study the Venus thermosphere up to at least $160 \mathrm{~km}$.

- The characteristics and trajectories of the charged particle producing aurora on the Venus nightside are still largely unexplored. The relation between the oxygen green line enhancement and the UV has not been firmly established and the impact of charged particle deposition on the local or global thermal balance still needs to be evaluated.

- Further results are expected from the cameras isolating different spectral windows on board the Akatsuki climate orbiter (Nakamura et al. 2011). Most instrumentation is designed to map infrared emissions from the lower atmosphere. Although not specifically intended to study the upper atmosphere, the Lightning and Airglow Camera (LAC) may provide observations of OI $557.7 \mathrm{~nm}$ and $\mathrm{O}_{2}$ Herzberg II nightside emissions.

- Developments of General Circulation Models extending from the ground to the upper atmosphere are underway. Once they reach an adequate level of reliability, they will remove the limitations set by the need for boundary questions that influence the dynamical and photochemical distribution of the atmospheric constituents.

Acknowledgements JCG thanks L. Soret for her help during the preparation of the manuscript. MALV was funded by the Spanish Ministry of Economy and Innovation and by the FEDER funds under grant ESP201565064-C2-1-P (MINECO/FEDER) as well as by CSIC “intramural” project 01450E022. The research effort of S. Bougher was funded in part by the NASA Venus Express Participating Scientist program through a subcontract from Southwest Research Institute (SwRI) to the University of Michigan. In addition, S. Bougher 
was also supported by the NASA Solar System Working Program through a subcontract from Bay Area Environmental Research Institute (BAERI) to the University of Michigan. This work was partly supported by the SCOOP/BRAIN research contract from the Belgian Federal Science Policy Office (BELSPO).

\section{References}

M.J. Alexander, A mechanism for the Venus thermospheric superrotation. Geophys. Res. Lett. 19, 2207-2210 (1992)

M.J. Alexander, A.I.F. Stewart, S.C. Solomon, S.W. Bougher, Local time asymmetries in the Venus thermosphere. J. Geophys. Res. 98, 10849-10871 (1993)

F. Altieri, F. Migliorini, L. Zasova, A. Shakun, G. Piccioni, G. Bellucci, Modeling VIRTIS/VEX O $2\left(a^{1} \Delta_{g}\right)$ nightglow profiles affected by the propagation of gravity waves in the Venus upper mesosphere. J. Geophys. Res., Planets 119, 2300-2316 (2014). doi:10.1002/2013JE004585

D.E. Anderson, The Mariner 5 ultraviolet photometer experiment: analysis of hydrogen Lyman alpha data. J. Geophys. Res. 81, 1213 (1976)

J. Bailey, S. Chamberlain, D. Crisp, V.S. Meadows, Near infrared imaging spectroscopy of Venus with the Anglo-Australian Telescope. Planet. Space Sci. 56, 1385-1390 (2008b)

J. Bailey, V.S. Meadows, S. Chamberlain, D. Crisp, The temperature of the Venus mesosphere from $\mathrm{O}_{2}\left(\mathrm{a}^{1} \Delta\right)$ airglow observations. Icarus 197, 247-259 (2008a)

P.M. Banks, G. Kockarts, Aeronomy (Elsevier, Amsterdam, 2013)

S.J. Bauer, L.H. Brace, D.M. Hunten, D.S. Intriligator, W.C. Knudsen, A.F. Nagy, C.T. Russell, F.L. Scarf, J.H. Wolfe, The Venus ionosphere and solar wind interaction. Space Sci. Rev. 20, 413-430 (1977)

J.L. Bertaux, J.E. Blamont, V.M. Lepine, V.G. Kurt, N.N. Romanova, A.S. Smirnov, Venera 11 and Venera 12 observations of e.u.v. emissions from the upper atmosphere of Venus. Planet. Space Sci. 29, 149-166 (1981). doi:10.1016/0032-0633(81)90029-5

J.-L. Bertaux, D. Nevejans, O. Korablev, E. Villard, E. Quémerais, E. Neefs, F. Montmessin, F. Leblanc, J.P. Dubois, E. Dimarellis, A. Hauchecorne, F. Lefèvre, P. Rannou, J.Y. Chaufray, M. Cabane, G. Cernogora, G. Souchon, F. Semelin, A. Reberac, E. Van Ransbeek, S. Berkenbosch, R. Clairquin, C. Muller, F. Forget, F. Hourdin, O. Talagrand, A. Rodin, A. Fedorova, A. Stepanov, I. Vinogradov, A. Kiselev, Y. Kalinnikov, G. Durry, B. Sandel, A. Stern, J.C. Gérard, SPICAV on Venus Express: three spectrometers to study the global structure and composition of the Venus atmosphere. Planet. Space Sci. 55, 12 (2007)

A. Bhardwaj, S.K. Jain, Calculations of $\mathrm{N}_{2}$ triplet states vibrational populations and band emissions in Venusian dayglow. Icarus 217, 2752-2758 (2012). doi:10.1016/j.icarus.2011.05.026

A. Bhardwaj, S.K. Jain, CO Cameron band and $\mathrm{CO}_{2}^{+} \mathrm{UV}$ doublet emissions in the dayglow of Venus: role of CO in the Cameron band production. J. Geophys. Res. 118, 3660-3671 (2013). doi:10.1002/jgra.50345

S.W. Bougher, W.J. Borucki, Venus $\mathrm{O}_{2}$ visible and IR nightglow: implications for lower thermosphere dynamics and chemistry. J. Geophys. Res. 99, 3759-3776 (1994)

S.W. Bougher, R.G. Roble, Comparative terrestrial planet thermospheres: 1. Solar cycle variation of global mean temperatures. J. Geophys. Res. 96, 11045-11055 (1991). doi:10.1029/91JA01162

S.W. Bougher, R.E. Dickinson, E.C. Ridley, R.G. Roble, A.F. Nagy, T. Cravens, Venus mesosphere and thermosphere II. Global circulation, temperature, and density variations. Icarus 68, 284-312 (1986)

S.W. Bougher, R.E. Dickinson, E.C. Ridley, R.G. Roble, Venus mesosphere and thermosphere III. Threedimensional general circulation with coupled dynamics and composition. Icarus 73, 545-573 (1988)

S.W. Bougher, D.M. Hunten, R.J. Phillips, Venus II (University of Arizona Press, Tucson, 1989). ISBN 9780-8165-1830-2

S.W. Bougher, J.C. Gérard, A.I.F. Stewart, C.G. Fesen, The Venus nitric oxide night airglow: model calculations based on the Venus Thermospheric General Circulation Model. J. Geophys. Res. 95(A5), 6271-6284 (1990)

S.W. Bougher, M.J. Alexander, H.G. Mayr, Upper Atmosphere Dynamics: Global Circulation and Gravity Waves, Venus II, Ch. 2.4 (U. of Arizona Press, Tucson, 1997), pp. 259-292

S.W. Bougher, S. Engel, R.G. Roble, B. Foster, Comparative terrestrial planet thermospheres: 2. Solar cycle variation of global structure and winds at equinox. J. Geophys. Res. 104, 16591-16611 (1999)

S.W. Bougher, S. Rafkin, P. Drossart, Dynamics of the Venus upper atmosphere: outstanding problems and new constraints expected from Venus Express. Planet. Space Sci. 54, 13-14 (2006). doi:10.1016/ j.pss.2006.04.023

S.W. Bougher, C.D. Parkinson, A.S. Brecht, J.-L. Fischer, A.-C. Vandaele, V. Wilquet, A. Mahieux, in Temperatures in Venus' Lower Thermosphere: Comparison of VTGCM and SOIR Profiles at the Terminator, SPICAM/SPICAV Team Meeting, Catania, Italy, 16-19 June (2013) 
S.W. Bougher, A.S. Brecht, R. Schulte, J.-L. Fischer, C.D. Parkinson, A. Mahieux, V. Wilquet, A.-C. Vandaele, Upper atmosphere temperature structure at the Venusian terminators: a comparison of SOIR and VTGCM results. Planet. Space Sci. (2015). doi:10.1016/j.pss.2015.01.012

L.H. Brace, A.J. Kliore, The structure of the Venus ionosphere. Space Sci. Rev. 55, 81-163 (1991)

L.H. Brace, J.P. Krehbiel, A.F. Nagy, T.M. Donahue, M.B. McElroy, A. Pedersen, R.F. Theis, Electron temperatures and densities in the Venus ionosphere-Pioneer Venus orbiter electron temperature probe results. Science 203, 763-765 (1979)

L.H. Brace, H.A. Taylor Jr., T.I. Gombosi, A.J. Kliore, W.C. Knudsen, A.F. Nagy, The ionosphere of Venus observations and their interpretation, in Venus, ed. by D.M. Hunten, L. Colin, T.M. Donahue, V.I. Moroz (1983), pp. 779-840

A.S. Brecht, S.W. Bougher, Dayside Thermal Structure of Venus' Upper Atmosphere Characterized by a Global Model. J. Geophys. Res. 117 (2012). doi:10.1029/2012JE004079

A.S. Brecht, S.W. Bougher, J.-C. Gérard, C.D. Parkinson, S. Rafkin, B. Foster, Understanding the variability of nightside temperatures, NO UV and $\mathrm{O}_{2}$ IR nightglow emissions in the Venus upper atmosphere. J. Geophys. Res. 116, E8004 (2011). doi:10.1029/2010je003770

A.S. Brecht, S.W. Bougher, J.-C. Gérard, L. Soret, Atomic oxygen distributions in the Venus thermosphere: comparisons between Venus Express observations and global model simulations. Icarus 217, 759-766 (2012). doi:10.1016/j.icarus.2011.06.033

T.K. Breus, K.I. Gringauz, M.I. Verigin, On the properties and origin of the Venus ionosphere. Adv. Space Res. 5, 145-156 (1985)

H.C. Brinton, H.A. Taylor Jr., H.B. Niemann, H.G. Mayr, A.F. Nagy, T.E. Cravens, D.F. Strobel, Venus nighttime hydrogen bulge. Geophys. Res. Lett. 7, 865-868 (1980)

E. Chassefiere, J.L. Bertaux, V.G. Kurt, A.S. Smirnov, Venus e.u.v. measurements of helium at $58.4 \mathrm{~nm}$ from Venera 11 and Venera 12 and implications for the outgassing history. Planet. Space Sci. 34, 585-602 (1986). doi:10.1016/0032-0633(86)90037-1

J.Y. Chaufray, J.-L. Bertaux, F. Leblanc, First observation of the Venus UV dayglow at limb from SPICAV/VEX. Geophys. Res. Lett. 39, L20201. (2012a). doi:10.1029/2012GL053626

J.Y. Chaufray, J.-L. Bertaux, E. Quémerais, E. Villard, F. Leblanc, Hydrogen density in the dayside Venusian exosphere derived from Lyman- $\alpha$ observations by SPICAV on Venus Express. Icarus 217, 767-778 (2012b)

R.T. Clancy, D.O. Muhleman, Diurnal CO variations in the Venus mesosphere from CO microwave spectra. Icarus 64(2), 157-182 (1985)

R.T. Clancy, D.O. Muhleman, Long-term (1979-1990) changes in the thermal, dynamical, and compositional structure of the Venus mesosphere as inferred from microwave spectral line observations of C-12O, C13O, and CO-18. Icarus 89, 129-146 (1991)

R.T. Clancy, B.J. Sandor, G.H. Moriarty-Schieven, Observational definition of the Venus mesopause: vertical structure, diurnal variation, and temporal instability. Icarus 161, 1-16 (2003). doi:10.1016/S00191035(02)00022-2

R.T. Clancy, B.J. Sandor, G.H. Moriarty-Schieven, Venus upper atmospheric CO, temperature, and winds across the afternoon/evening terminator from June 2007 JCMT sub-millimeter line observations. Planet. Space Sci. 56, 1344-1354 (2008). doi:10.1016/j.pss/2008.05.007

R.T. Clancy, B.J. Sandor, A. García-Muñoz, F. Lefèvre, M.D. Smith, M.J. Wolff, F. Montmessin, S.L. Murchie, H. Nair, First detection of Mars atmospheric hydroxyl: CRISM near-IR measurement versus LMD GCM simulation of $\mathrm{OH}$ Meinel band emission in the Mars polar winter atmosphere. Icarus 226, 272-281 (2013)

A. Collet, C. Cox, J.-C. Gérard, Two-dimensional time-dependent model of the transport of minor species in the Venus night side upper atmosphere. Planet. Space Sci. 58, 14-15 (2010). doi:10. 1016/j.pss.2010.08.016

P. Connes, J.F. Noxon, W.A. Traub, N.P. Carleton, $\mathrm{O}_{2}\left({ }^{1} \Delta\right)$ emission in the day and night airglow of Venus. Astrophys. J. 233, L29-L32 (1979)

C. Cox, J.-C. Gérard, B. Hubert, J.L. Bertaux, S.W. Bougher, The Mars ultraviolet dayglow variability: SPICAM observations and comparison with airglow model. J. Geophys. Res. 115, E04010 (2010). doi:10.1029/2009JE003504

T.E. Cravens, J.U. Kozyra, A.F. Nagy, A.J. Kliore, The ionospheric peak on the Venus dayside. J. Geophys. Res. 86, 11323-11329 (1981)

D. Crisp, V.S. Meadows, B. Bézard, C. deBergh, J.-P. Maillard, F.P. Mills, Ground-based near-infrared observations of the Venus nightside: $1.27-\mu \mathrm{m} \mathrm{O}_{2}\left(\mathrm{a}^{1} \Delta_{\mathrm{g}}\right)$ airglow from the upper atmosphere. J. Geophys. Res. 101, 4577-4594 (1996). doi:10.1029/95JE03136

J. Crovisier, E. Lellouch, C. deBergh, J.-P. Maillard, B.L. Lutz, B. Bézard, Carbon monoxide emissions at $4.7 \mu \mathrm{m}$ from Venus atmosphere. Planet. Space Sci. 54, 1398-1414 (2006) 
A.D. Del Genio, W.B. Rossow, Temporal variability of UV cloud features in the Venus stratosphere. Icarus 51, 391-415 (1982)

A.D. Del Genio, W.B. Rossow, Planetary-scale waves and the cyclic nature of cloud top dynamics on Venus. J. Atmos. Sci. 47, 293-318 (1990)

D. Deming et al., Observations of the 10- $\mu$ m natural laser emission from the mesospheres of Mars and Venus. Icarus 55, 347-355 (1983)

R.E. Dickinson, Infrared radiative HeatingCooling in the Venusian mesosphere. I: Global mean radiative equilibrium. J. Atmos. Sci. 29, 1531-1556 (1972). doi:10.1175/1520-0469

T.M. Donahue, D.H. Grinspoon, R.E. Hartle, R.R. Hodgee, Ion/neutral escape of hydrogen deuterium: evolution of water, in Venus H - Geology, Geophysics, Atmosphere, Solar Wind Environment, ed. by S.W. Bougher, D.M. Hunten, R.J. Philips (Univ. of Ariz. Press, Tuscon, 1997), pp. 385-414

P. Drossart et al., A dynamic upper atmosphere of Venus as revealed by VIRTIS on Venus Express. Nature 450, 7170 (2007). doi:10.1038/nature06140

P.D. Feldman, E.B. Burgh, S.T. Durrance, A.F. Davidsen, Far-ultraviolet spectroscopy of Venus and Mars at $4 \AA$ resolution with the Hopkins ultraviolet telescope on Astro-2. Astrophys. J. 538, 395 (2000). doi:10.1086/309125

G. Fjeldbo, V.R. Eshleman, Atmosphere of Venus as studied with the Mariner 5 dual radio-frequency occultation experiment. Radio Sci. 4, 879-897 (1969)

G. Fjeldbo, B. Seidel, D. Sweetnam, H.T. Howard, The Mariner 10 radio occultation measurements of the ionosphere of Venus. J. Atmos. Sci. 32, 1232-1236 (1975)

J.M. Forbes, M.E. Hagan, Diurnal Kelvin wave in the atmosphere of mars: towards an understanding of 'stationary' density structures observed by the MGS accelerometer. Geophys. Res. Lett. 27, 3563-3566 (2000)

J.M. Forbes, A. Konopliv, Oscillation of Venus' upper atmosphere. Geophys. Res. Lett. 34, L08202 (2007). doi:10.1029/2007GL029252

J.L. Fox, Airglow and Aurora in the atmospheres of Venus and Mars, in Venus and Mars: Atmospheres, Ionospheres, and Solar Wind Interactions, ed. by e.J.G. Luhmann, M. Tatrallyay, R.O. Pepin (American Geophysical Union, Washington, 1992). doi:10.1029/GM066p0191

J.L. Fox, Near-terminator Venus ionosphere: how Chapman-esque? J. Geophys. Res. 112, E04S02 (2007). doi:10.1029/2006JE002736

J.L. Fox, The ionospheric source of the red and green lines of atomic oxygen in the Venus nightglow. Icarus 221, 787-799 (2012)

J.L. Fox, S.W. Bougher, Structure, luminosity, and dynamics of the Venus thermosphere, in Venus Aeronomy (Springer, Netherlands, 1991), pp. 357-489

J.L. Fox, A. Dalgarno, Ionization, luminosity, and heating of the upper atmosphere of Mars. J. Geophys. Res. 84(A12), 7315-7333 (1979). doi:10.1029/JA084iA12p07315

J.L. Fox, A.B. Hać, Spectrum of hot O at the exobases of the terrestrial planets. J. Geophys. Res. 102(A11), 24005 (1997)

J.L. Fox, N.E.F. Hać, Intensities of the Venusian $\mathrm{N}_{2}$ electron-impact excited dayglow emissions. J. Geophys. Res. 118, 7850-7863 (2013). doi:10.1002/2013JA019277

J.L. Fox, L.J. Paxton, $\mathrm{CC}^{+}$in the Venusian thermosphere/ionosphere. J. Geophys. Res. 110, A01311 (2005). doi:10.1029/2004JA010813

J.L. Fox, A.I.F. Stewart, The Venus ultraviolet aurora: a soft electron source. J. Geophys. Res. 96, 9829-9832 (1991)

J.L. Fox, K.Y. Sung, Solar activity variations of the Venus thermosphere/ionosphere. J. Geophys. Res. 106, 21305-21336 (2001)

Y. Futaana, G.S. Wieser, S. Barabash, J.G. Luhmann, Solar wind interaction and impact on the Venus atmosphere. Space Sci. Rev. 1-57 (2017). doi:10.1007/s11214-017-0362-8

Y. Futaana, J.Y. Chaufray, H.T. Smith, P. Garnier, H. Lichtenegger, M. Delva, H. Gröller, A. Mura, Exospheres and energetic neutral atoms of Mars, Venus and Titan. Space Sci. Rev. 162, 213-266 (2011). doi:10.1007/s11214-011-9834-4

R.F. Garcia, P. Drossart, G. Piccioni, M. López-Valverde, G. Occhipinti, Gravity waves in the upper atmosphere of Venus revealed by $\mathrm{CO}_{2}$ nonlocal thermodynamic equilibrium emissions. J. Geophys. Res. 114, E00B32 (2009). doi:10.1029/2008JE003073

A. García-Muñoz, F.P. Mills, G. Piccioni, P. Drossart, The near-infrared nitric oxide nightglow in the upper atmosphere of Venus. Proc. Natl. Acad. Sci. USA 106, 985-988 (2009a)

A. Garcia-Muñoz, F.P. Mills, T.G. Slanger, G. Piccioni, P. Drossart, Visible and near-infrared nightglow of molecular oxygen in the atmosphere of Venus. J. Geophys. Res. 114, E12002 (2009b). doi:10.1029/ 2009JE003447

A. García-Muñoz, R. Hueso, A. Sánchez-Lavega, W.J. Markiewicz, D.V. Titov, O. Witasse, A. Opitz, Limb imaging of the Venus $\mathrm{O}_{2}$ visible nightglow with the Venus Monitoring Camera. Geophys. Res. Lett. 40, 2539-2543 (2013). doi:10.1002/grl.50553 
J.-C. Gérard, I.A.F. Stewart, S. Bougher, The altitude distribution of the Venus ultraviolet nightglow and its implications on vertical transport properties. Geophys. Res. Lett. 8, 633-636 (1981)

J.-C. Gérard, C. Cox, A. Saglam, J.L. Bertaux, E. Villard, C. Nehme, Limb observations of the ultraviolet nitric oxide nightglow with SPICAV on board Venus Express. J. Geophys. Res. 113, E00b03 (2008b). doi:10.1029/2008je003078

J.-C. Gérard, B. Hubert, V.I. Shematovich, D.V. Bisikalo, G.R. Gladstone, The Venus ultraviolet oxygen dayglow and aurora: model comparison with observations. Planet. Space Sci. 56, 542-552 (2008a). doi:10.1016/j.pss.2007.11.008

J.-C. Gérard, A. Saglam, G. Piccioni, P. Drossart, C. Cox, S. Erard, R. Hueso, A. Sanchez-Lavega, Distribution of the $\mathrm{O}_{2}$ infrared nightglow observed with VIRTIS on board Venus Express. Geophys. Res. Lett. 35, L02207 (2008c). doi:10.1029/2007g1032021

J.-C. Gérard, A. Saglam, G. Piccioni, P. Drossart, F. Montmessin, J.L. Bertaux, Atomic oxygen distribution in the Venus mesosphere from observations of $\mathrm{O}_{2}$ infrared airglow by VIRTIS-Venus Express. Icarus 199, 264-272 (2009a). doi:10.1016/j.icarus.2008.09.016

J.-C. Gérard, C. Cox, L. Soret, A. Saglam, G. Piccioni, J.-L. Bertaux, P. Drossart, Concurrent observations of the ultraviolet nitric oxide and infrared $\mathrm{O}_{2}$ nightglow emissions with Venus Express. J. Geophys. Res. 114, E00B44 (2009b). doi:10.1029/2009JE003371

J.-C. Gérard, L. Soret, A. Saglam, G. Piccioni, P. Drossart, The distributions of the OH Meinel and $\mathrm{O}_{2}\left(\mathrm{a}^{1} \Delta-\right.$ $\mathrm{X}^{3} \Sigma$ ) nightglow emissions in the Venus mesosphere based on VIRTIS observations. Adv. Space Res. 45, 1268-1275 (2010). doi:10.1016/j.asr.2010.01.022

J.-C. Gérard, B. Hubert, J. Gustin, V.I. Shematovich, D. Bisikalo, G.R. Gladstone, L.W. Esposito, EUV spectroscopy of the Venus dayglow with UVIS on Cassini. Icarus 211, 70-80 (2011a). doi:10.1016/ j.icarus.2010.09.020

J.-C. Gérard, J. Gustin, B. Hubert, G.R. Gladstone, L.W. Esposito, Measurements of the helium $584 \AA$ airglow during the Cassini flyby of Venus. Planet. Space Sci. 59, 1524-1528 (2011b)

J.-C. Gérard, L. Soret, G. Piccioni, P. Drossart, Spatial correlation of OH Meinel and $\mathrm{O}_{2}$ infrared atmospheric nightglow emissions observed with VIRTIS-M on board Venus Express. Icarus 217, 813-817 (2012). doi:10.1016/j.icarus.2011.09.010

J.-C. Gérard, L. Soret, A. Migliorini, G. Piccioni, Oxygen nightglow emissions of Venus: vertical distribution and collisional quenching. Icarus 223, 602-608 (2013). doi:10.1016/j.icarus.2012.11.019

J.-C. Gérard, L. Soret, G. Piccioni, P. Drossart, Latitudinal structure of the Venus $\mathrm{O}_{2}$ infrared airglow: a signature of small-scale dynamical processes in the upper atmosphere. Icarus 236, 93-103 (2014). doi:10.1016/j.icarus.2014.03.028

P.J. Gierasch, R.M. Goody, R.E. Young, D. Crisp, C. Edwards, R. Kahn, D. Rider, A. Del Genio, R. Greeley, A. Hou, C.B. Leovy, D. McCleese, M. Newman, The general circulation of the Venus atmosphere: an assessment, in Venus II-Geology, Geophysics, Atmosphere, and Solar Wind Environment, ed. by S.W. Bougher, D.M. Hunten, R.J. Phillips (Univ. of Ariz. Press, Tuscon, 1997), pp. 459-500

G. Gilli, Carbon monoxide and temperature in the upper atmosphere of Venus through the analysis of limb observations by VIRTIS/Venus Express. Ph.D thesis, Universidad de Granada, Spain (2012)

G. Gilli, M.A. López-Valverde, P. Drossart, G. Piccioni, S. Erard, A. Cardesín, Moinelo Limb observations of $\mathrm{CO}_{2}$ and $\mathrm{CO}$ non-LTE emissions in the Venus atmosphere by VIRTIS/Venus Express. J. Geophys. Res. 114, E00B29 (2009). doi:10.1029/2008JE003112

G. Gilli, M.A. López-Valverde, B. Funke, M. López-Puertas, P. Drossart, G. Piccioni, V. Formisano, NonLTE CO limb emission at $4.7 \mu \mathrm{m}$ in the upper atmosphere of Venus, Mars and Earth: observations and modeling. Planet. Space Sci. 59, 1010-1018 (2011). doi:10.1016/j.pss.2010.07.023

G. Gilli, M.A. López-Valverde, J. Peralta, S. Bougher, A. Brecht, P. Drossart, G. Piccioni, Carbon monoxide and temperature in the upper atmosphere of Venus from VIRTIS/Venus Express non-LTE limb measurements. Icarus 248, 478-498 (2015). doi:10.1016/j.icarus.2014.10.047

G. Gilli, S. Lebonnois, F. González-Galindo, M.A. López-Valverde, A. Stolzenbach, F. Lefèvre, J.Y. Chaufray, F. Lott, Thermal structure of the upper atmosphere of Venus simulated by a ground-tothermosphere GCM. Icarus 281, 55-72 (2017)

Z. Girazian, P. Withers, B. Häusler, M. Pätzold, S. Tellmann, K. Peter, Characterization of the lower layer in the dayside Venus ionosphere and comparisons with Mars. Planet. Space Sci. 117, 146-158 (2015)

J.J. Goldstein, M.J. Mumma, T. Kostiuk, D. Deming, F. Espenak, D. Zipoy, Absolute wind velocities in the lower thermosphere of Venus using infrared heterodyne spectroscopy. Icarus 94, 45-63 (1991)

B.F. Gordiets, V.I. Panchenko, Nonequilibrium infrared emission and the natural laser effect in the Venus and Mars atmospheres. Cosm. Res. 34, 721-734 (1983)

C.L. Gray, N.J. Chanover, T.G. Slanger, K. Molaverdikhani, The effect of solar flares, coronal mass ejections, and solar wind streams on Venus' $5577 \AA$ Å oxygen green line. Icarus 233, 342-347 (2014). doi:10.1016/ j.icarus.2014.01.029 
J.M. Grebowsky, J.I. Moses, W.D. Pesnell, Meteoric material, an important component of planetary atmospheres. Washington DC American Geophysical Union Geophysical Monograph Series 130, 235 (2002)

H. Gröller, V.I. Shematovich, H.I.M. Lichtenegger, H. Lammer, M. Pfleger, Y.N. Kulikov, W. Macher, U.V. Amerstorfer, H.K. Biernat, Venus' atomic hot oxygen environment. J. Geophys. Res. 115, E12017 (2010). doi:10.1029/2010JE003697

H. Gröller, H. Lammer, H.I.M. Lichtenegger, M. Pfleger, O. Dutuit, V.I. Shematovich, Y.N. Kulikov, H.K. Biernat, Hot oxygen atoms in the Venus nightside exosphere. Geophys. Res. Lett. 39, L03202 (2012). doi:10.1029/2011GL050421

G. Gronoff, J. Lilensten, C. Simon, M. Barthélemy, F. Leblanc, O. Dutuit, Modeling the Venus airglow. Astron. Astrophys. 482, 1015-1029 (2008)

S. Gulkis, R.K. Kakar, M.J. Klein, E.T. Olsen, W.J. Wilson, Venus: detection of variations in stratospheric carbon monoxide. in Planetary Atmospheres: Nineteenth Symposium of the Royal Society of Canada, ed. by A.V. Jones (Royal Society of Canada, Ottawa, 1977), p. 61

M.A. Gurwell, D.O. Muhleman, K.P. Shah, G.L. Berge, D.J. Rudy, A.W. Grossman, Observations of the CO bulge on Venus and implications for mesospheric winds. Icarus 115, 141-158 (1995)

B. Häusler, M. Pätzold, G.L. Tyler, R.A. Simpson, M.K. Bird, V. Dehant, J.P. Barriot, W. Eidel, R. Mattei, S. Remus, J. Selle, S. Tellmann, T. Imamura, Radio science investigations by VeRa onboard the Venus Express spacecraft. Planet. Space Sci. 54, 1315-1335 (2006)

A.E. Hedin, H.B. Niemann, W.T. Kasprzak, A. Seiff, Global empirical model of the Venus thermosphere. J. Geophys. Res. 88, A1 (1983)

R.R. Hodges, An exospheric perspective of isotopic fractionation of hydrogen on Venus. J. Geophys. Res. 104, 8463-8471 (1999)

R.R. Hodges, Distributions of hot oxygen for Venus and Mars. J. Geophys. Res. 105, 6971-6981 (2000). doi:10.1029/1999JE001138

C.W. Hord et al., Galileo ultraviolet spectrometer experiment: initial Venus and interplanetary cruise results. Science 253(5027), 1548-1550 (1991). doi:10.1126/science.253.5027.1548

N. Hoshino, H. Fujiwara, M. Takagi, Y. Takahashi, Y. Kasaba, Characteristics of planetary-scale waves simulated by a new Venusian mesosphere and thermosphere general circulation model. Icarus 217, 818-830 (2012). doi:10.1016/j.icarus.2011.06.039

H.T. Howard et al., Venus: Mass, gravity field, atmosphere, and ionosphere as measured by the Mariner 10 dual-frequency radio system. Science 183(4131), 1297-1301 (1974)

B. Hubert, J.C. Gérard, J. Gustin, V.I. Shematovich, D.V. Bisikalo, A.I. Stewart, G.R. Gladstone, UVIS observations of the FUV OI and CO 4P Venus dayglow during the Cassini flyby. Icarus 207, 549-557 (2010). doi:10.1016/j.icarus.2009.12.029

B. Hubert, J.-C. Gérard, J. Gustin, D.V. Bisikalo, V.I. Shematovich, G.R. Gladstone, Cassini-UVIS observation of dayglow FUV emissions of carbon in the thermosphere of Venus. Icarus 220, 635-646 (2012). doi:10.1016/j.icarus.2012.06.002

R.A. Hueso, A. Sánchez-Lavega, G. Piccioni, P. Drossart, J.-C. Gérard, I. Khatuntsev, L. Zasova, A. Migliorini, Morphology and dynamics of Venus oxygen airglow from Venus Express/Visible and Infrared Thermal Imaging Spectrometer observations. J. Geophys. Res. 113, E00B02 (2008). doi:10.1029/2008JE003081

D.L. Huestis, S.W. Bougher, J.L. Fox, M. Galand, R.E. Johnson, J.I. Moses, J.C. Pickering, Cross sections and reaction rates for comparative planetary aeronomy. Space Sci. Rev. 139, 63-105 (2008). doi:10.1007/s11214-008-9383-7

J.M. Jenkins, P.G. Steffes, D.P. Hinson, J.D. Twicken, G.L. Tyler, Radio occultation studies of the Venus atmosphere with the Magellan spacecraft 2: results from the October 1991 experiments. Icarus 110, 79-94 (1994)

M.A. Johnson, A.L. Betz, R.A. McLaren, C.H. Townes, E.C. Sutton, Nonthermal 10 micron $\mathrm{CO}_{2}$ emission lines in the atmospheres of Mars and Venus. Astrophys. J. 208, L145-L148 (1976). doi:10.1086/182252

H.U. Kaeufl, H. Rothermel, S. Drapatz, Investigation of the Martian atmosphere by 10 micron heterodyne spectroscopy. Astron. Astrophys. 136, 319-325 (1984)

E. Kallio, J.Y. Chaufray, R. Modolo, D. Snowden, R. Winglee, Modeling of Venus, Mars, and Titan. Space Sci. Rev. 162, 267-307 (2011). doi:10.1007/s11214-011-9814-8

W.T. Kasprzak, H.B. Niemann, A.E. Hedin, S.W. Bougher, Wave-like perturbations observed at low altitudes by the Pioneer Venus Orbiter Neutral Mass Spectrometer during Orbiter entry. Geophys. Res. Lett. 20, 2755-2758 (1993)

W.T. Kasprzak, G.M. Keating, N.C. Hsu, A.I.F. Stewart, W.B. Colwell, S.W. Bougher, Solar activity behavior of the thermosphere, in Venus II (U. of Arizona Press, Tucson, 1997), p. 225

G.M. Keating, J.Y. Nicholson III., L.R. Lake, Venus upper atmosphere structure. J. Geophys. Res. 85, 79417956 (1980) 
D. Kella, P.J. Johnson, H.B. Pedersen, L. Vejby-Christenson, L.H. Andersen, The source of green light emission determined from a heavy-ion storage ring experiment. Science 276, 1530-1533 (1997)

A.J. Kliore, Recent results on the Venus atmosphere from Pioneer Venus radio-occultations. Adv. Space Res. 5, 41-49 (1985)

A.J. Kliore, Radio occultation observations of the ionospheres of Mars and Venus, in Venus and Mars: Atmospheres, Ionospheres and Solar Wind Interaction. AGU Geophysical Monograph, vol. 66 (1992)

A.J. Kliore, J.G. Luhmann, Solar cycle effects on the structure of the electron density profiles in the dayside ionosphere of Venus. J. Geophys. Res. 96, 21281 (1991)

A.J. Kliore, G.S. Levy, D.L. Cain, G. Fjeldbo, S.I. Rasool, Atmosphere and ionosphere of Venus from the Mariner V S-band radio occultation measurement. Science 158, 1683-1688 (1967)

A.J. Kliore, I.R. Patel, A.F. Nagy, T.E. Cravens, T.I. Gombosi, Initial observations of the nightside ionosphere of Venus from Pioneer Venus Orbiter radio occultations. Science 205, 99-102 (1979a)

A.J. Kliore, R. Woo, J.W. Armstrong, I.R. Patel, T.A. Croft, The polar ionosphere of Venus near the terminator from early Pioneer Venus orbiter radio occultations. Science 203, 765-768 (1979b)

A.J. Kliore, V.I. Moroz, G.M. Keating, The Venus international reference atmosphere, in Adv. Space Res., vol. 5 (Pergamon Press, Elmsford, 1985), pp. 1-305

A.J. Kliore, J.G. Luhmann, M.H.G. Zhang, The effect of the solar cycle on the maintenance of the nightside ionosphere of Venus. J. Geophys. Res. 96, 11065 (1991)

W.C. Knudsen, K.L. Miller, Pioneer Venus suprathermal electron flux measurements in the Venus umbra. J. Geophys. Res. 90, 2695-2702 (1985). doi:10.1029/JA090iA03p02695

W.C. Knudsen, K. Spenner, R.C. Whitten, J.R. Spreiter, K.L. Miller, V. Novak, Thermal structure and major ion composition of the Venus ionosphere: First RPA results from Venus orbiter. Science 203, 757-763 (1979a)

W.C. Knudsen, K. Spenner, R.C. Whitten, J.R. Spreiter, K.L. Miller, V. Novak, Thermal structure and energy influx to the day-and nightside Venus ionosphere. Science 205, 105-107 (1979b)

V.A. Krasnopolsky, Venus spectroscopy in the 3000-8000 A region by Veneras 9 and 10, in Venus, ed. by D.M. Hunten et al. (The University of Arizona Press, Tucson, 1983), pp. 459-483

V.A. Krasnopolsky, Oxygen emissions in the night airglow of the Earth, Venus and Mars. Planet. Space Sci. 34, 511-518 (1986)

V.A. Krasnopolsky, Venus night airglow: ground-based detection of $\mathrm{OH}$, observations of $\mathrm{O}_{2}$ emissions, and photochemical model. Icarus 207, 17-27 (2010). doi:10.1016/j.icarus.2009.10.019

V.A. Krasnopolsky, Excitation of the oxygen nightglow on the terrestrial planets. Planet. Space Sci. 59, 754766 (2011)

V.A. Krasnopolsky, Nightside photochemical model and night airglow on Venus. Planet. Space Sci. 85, 78-88 (2013). doi:10.1016/j.pss.2013.05.022

V.A. Krasnopolsky, Observations of $\mathrm{CO}$ dayglow at $4.7 \mu \mathrm{m}, \mathrm{CO}$ mixing ratios, and temperatures at 74 and 104-111 km on Venus. Icarus 237, 340-349 (2014a). doi:10.1016/j.icarus.2014.04.043

V.A. Krasnopolsky, Observations of the $\mathrm{CO}$ dayglow at $4.7 \mu \mathrm{m}$ on Mars: variations of temperature and $\mathrm{CO}$ mixing ratio at $50 \mathrm{~km}$. Icarus 228, 189-196 (2014b)

V.A. Krasnopolsky, G.R. Gladstone, Helium on Mars and Venus: EUVE observations and modeling. Icarus 176(2), 395-407 (2005)

V.A. Krasnopolsky, V.A. Parshev, Photochemistry of the Venus atmosphere, in Venus, ed. by D.M. Hunten, L. Colin, T.M. Donahue, V.I. Moroz (The University of Arizona Press, Tucson, 1983), pp. 459-483

M.A. Krestyanikova, V.I. Shematovich, Stochastic models of hot planetary and satellite coronas: a hot oxygen corona of Mars. Sol. Syst. Res. 40, 384-392 (2006). doi:10.1134/S0038094606050030

S. Kumar, A.L. Broadfoot, He $584 \AA$ airglow emission from Venus: Mariner 10 observations. Geophys. Res. Lett. 2, 357-360 (1975). doi:10.1029/GL002i008p00357

H. Lammer et al., Loss of hydrogen and oxygen from the upper atmosphere of Venus. Planet. Space Sci. 54, 1445-1456 (2006). doi:10.1016/j.pss.2006.04.022

S. Lebonnois et al., Superrotation of Venus' atmosphere analysed with a full general circulation model. J. Geophys. Res. 115, E06006 (2010). doi:10.1029/2009JE003458

F. Leblanc, J.Y. Chaufray, J.L. Bertaux, On Martian nitrogen dayglow emission observed by SPICAM UV spectrograph/Mars Express. Geophys. Res. Lett. 34, L02206 (2007). doi:10.1029/2006GL028437

E. Lellouch, J.J. Goldstein, J. Rosenqvist, S.W. Bougher, G. Paubert, Global circulation, thermal structure, and carbon monoxide distribution in Venus' mesosphere in 1991. Icarus 110, 315-339 (1994)

E. Lellouch, R.T. Clancy, D. Crisp, A. Kliore, D. Titov, S.W. Bougher, Monitoring of mesospheric structure and dynamics, in Venus II (U. of Arizona Press, Tucson, 1997), pp. 295-324

E. Lellouch, G. Paubert, R. Moreno, A. Moullet, Monitoring Venus' mesospheric winds in support of Venus Express: IRAM 30-m and APEX observations. Planet. Space Sci. 56, 1355-1367 (2008). doi:10.1016/ j.pss.2008.06.010 
H.I.M. Lichtenegger, H. Gröller, H. Lammer, Yu.N. Kulikov, V.I. Shematovich, On the elusive hot oxygen corona of Venus. Geophys. Res. Lett. 36, L10204 (2009). doi:10.1029/2009GL037575

S. Limaye et al., The thermal structure of the Venus atmosphere: intercomparison of Venus Express and ground based observations of vertical temperature and density profiles. Icarus 294, 124-155 (2017)

V.M. Linkin et al., VEGA balloon dynamics and vertical winds in the Venus middle cloud region. Science 231, 1417-1419 (1986)

M.A. López-Puertas, F.W. Taylor, Non-LTE Radiative Transfer in the Atmosphere (World Scientific Publishing Co., Singapore, 2001)

M.A. López-Valverde, M. López-Puertas, J.J. López-Moreno, V. Formisano, D. Grassi, A. Maturilli, E. Lellouch, P. Drossart, Analysis of $\mathrm{CO}_{2}$ non-LTE emissions at $4.3 \mu \mathrm{m}$ in the Martian atmosphere as observed by PFS/Mars Express and SWS/ISO. Planet. Space Sci. 53, 1079-1087 (2005). doi:10.1016/j.pss.2005.03.007

M.A. López-Valverde, P. Drossart, R. Carlson, R. Mehlman, M. Roos-Serote, Non-LTE infrared observations at Venus: From NIMS/GALILEO to VIRTIS/Venus Express. Planet. Space Sci. 55, 1757-1771 (2007). doi:10.1016/j.pss.2007.01.008

M.A. López-Valverde, M. López-Puertas, B. Funke, G. Gilli, M. García-Comas, P. Drossart, G. Piccioni, V. Formisano, Modelling the atmospheric limb emission of $\mathrm{CO}_{2}$ at $4.3 \mu \mathrm{m}$ in the terrestrial planets. Planet. Space Sci. 59, 988-998 (2011a). doi:10.1016/j.pss.2010.02.001

M.A. López-Valverde, G. Sonnabend, M. Sornig, P. Kroetz, Modelling the atmospheric $\mathrm{CO}_{2}$ 10- $\mu$ m nonthermal emission in Mars and Venus at high spectral resolution. Planet. Space Sci. 59, 999-1009 (2011b). doi:10.1016/j.pss.2010.11.011

R. Lundin, S. Barabash, Y. Futaana, M. Holmström, H. Perez-de-Tejada, J.-A. Sauvaud, A large-scale flow vortex in the Venus plasma tail and its fluid dynamic interpretation. Geophys. Res. Lett. 40, 1273-1278 (2013). doi:10.1002/grl.50309

Mariner Stanford Group, Venus: IonosphereAtmosphere as measured by dual-frequency radio occultation of Mariner V. Science 158, 1678-1683 (1967)

S.T. Massie, D.M. Hunten, D.R. Sowell, Day and night models of the Venus thermosphere. J. Geophys. Res. 88, 3955-3969 (1983). doi:10.1029/JA088iA05p03955

K. Masunaga, K. Seki, N. Terada, F. Tsuchiya, T. Kimura, K. Yoshioka, G. Murakami, A. Yamazaki, M. Kagitani, C. Tao, A. Fedorov, Y. Futaana, T.L. Zhang, D. Shiota, F. Leblanc, J.-Y. Chaufray, I. Yoshikawa, Periodic variations of oxygen EUV dayglow in the upper atmosphere of Venus: Hisaki/EXCEED observations. J. Geophys. Res. 120, 2037 (2015). doi:10.1002/2015JE004849

K. Masunaga, K. Seki, N. Terada, F. Tsuchiya, T. Kimura, K. Yoshioka, G. Murakami, A. Yamazaki, C. Tao, F. Leblanc, I. Yoshikawa, Dawn-dusk difference of periodic oxygen EUV dayglow variations at Venus observed by Hisaki. Icarus 292, 102-110 (2017). doi:10.1016/j.icarus.2016.12.027

H.G. Mayr, I. Harris, H.B. Niemann, H.C. Brinton, N.W. Spencer, H.A. Taylor, R.E. Hartle, W.R. Hoegy, D.M. Hunten, Dynamic properties of the thermosphere inferred from Pioneer Venus mass spectrometer measurements. J. Geophys. Res. 85, 7841-7847 (1980). doi:10.1029/JA085i.A13p07841

M.B. McElroy, M.J. Prather, J.M. Rodriguez, Escape of hydrogen from Venus. Science 215, 1614-1615 (1982). doi:10.1126/science.215.4540.1614

J.G. Mengel, H.G. Mayr, I. Harris, D.R. Stevens-Rayburn, Non-linear three-dimensional spectral model of the Venusian thermosphere with superrotation: II. Temperature, composition, and winds. Planet. Space Sci. 37, 707-722 (1989)

A. Migliorini, G. Piccioni, A. Cardesín-Moinelo, P. Drossart, Hydroxyl airglow on Venus in comparison with Earth. Planet. Space Sci. 59, 974-980 (2011). doi:10.1016/j.pss.2010.05.004

A. Migliorini, G. Piccioni, J.C. Gérard, L. Soret, T.G. Slanger, R. Politi, M. Snels, P. Drossart, F. Nuccilli, The characteristics of the $\mathrm{O}_{2}$ Herzberg II and Chamberlain bands observed with VIRTIS/Venus Express. Icarus 223, 609-614 (2013a). doi:10.1016/j.icarus.2012.11.017

A. Migliorini, G. Piccioni, F. Capaccioni, G. Filacchione, F. Tosi, J.C. Gérard, Comparative analysis of airglow emissions in terrestrial planets, observed with VIRTIS-M instruments on board Rosetta and Venus Express. Icarus 226, 1115-1127 (2013b). doi:10.1016/j.icarus.2013.07.027

A. Migliorini, M. Snels, J.C. Gérard, L. Soret, G. Piccioni, P. Drossart, Temperature estimation from hydroxyl airglow emission in the Venus night side mesosphere. Icarus 300, 386-391 (2018). doi:10.1016/j.icarus.2017.09.026

J.G. Molina-Cuberos, J.J. López-Moreno, F. Arnold, Meteoric layers in planetary atmospheres, in Planetary Atmospheric Electricity (Springer, New York, 2008), pp. 175-191

F. Montmessin, J.L. Bertaux, F. Lefèvre, E. Marcq, D. Belyaev, J.C. Gérard, O. Korablev, A. Fedorova, V. Sarago, A.C. Vandaele, A layer of ozone detected in the nightside upper atmosphere of Venus. Icarus 216, 1 (2011). doi:10.1016/j.icarus.2011.08.010

M.J. Mumma, D. Buhl, G. Chin, D. Deming, F. Espenak, T. Kostiuk, D. Zipoy, Discovery of natural gain amplification in the 10-micrometer carbon dioxide laser bands on Mars: a natural laser. Science 212, 45-49 (1981). doi:10.1126/science.212.4490.45 
A.F. Nagy, T.E. Cravens, Hot oxygen atoms in the upper atmospheres of Venus and Mars. Geophys. Res. Lett. 15, 433-435 (1988)

A.F. Nagy, T.E. Cravens, J.H. Yee, A.I.F. Stewart, Hot oxygen atoms in the upper atmosphere of Venus. Geophys. Res. Lett. 8, 629-632 (1981)

A.F. Nagy, J. Kim, T.E. Cravens, Hot hydrogen and oxygen atoms in the upper atmospheres of Venus and Mars. Ann. Geophys. 8, 251-256 (1990)

H. Nakagawa, N. Hoshino, M. Sornig, Y. Kasaba, G. Sonnabend, D. Stupar, S. Aoki, I. Murata, Comparison of general circulation model atmospheric wave simulations with wind observations of Venusian mesosphere. Icarus 225, 840-849 (2013)

M. Nakamura et al., Overview of Venus orbiter Akatsuki. Earth Planets Space 63(5), 443-457 (2011). doi:10.5047/eps.2011.02.009

H.B. Niemann, R.E. Hartle, A.E. Hedin, W.T. Kasprzak, N.W. Spencer, D.M. Hunten, G.R. Carignan, Venus upper atmosphere neutral gas composition: first observations of the diurnal variations. Science $\mathbf{2 0 5}$, 54-56 (1979)

H.B. Niemann, W.T. Kasprzak, A.E. Hedin, D.M. Hunten, N.W. Spencer, Mass spectrometer measurements of the neutral gas composition of the thermosphere and exosphere of Venus. J. Geophys. Res. 85, 78177827 (1980). doi:10.1029/JA085iA13p07817

S. Ohtsuki, N. Iwagami, H. Sagawa, Y. Kasaba, M. Ueno, T. Imamura, Ground-based observation of the Venus 1.27- $\mathrm{m} \mathrm{O}_{2}$ airglow. Adv. Space Res. 36, 2038-2042 (2005). doi:10.1016/j.asr.2005.05.078

S. Ohtsuki, N. Iwagami, H. Sagawa, M. Ueno, Y. Kasaba, T. Imamura, K. Yanagisawa, E. Nishihara, Distributions of the Venus 1.27- $\mu \mathrm{m} \mathrm{O}_{2}$ airglow and rotational temperature. Planet. Space Sci. 56, 1391-1398 (2008). doi:10.1016/j.pss.2008.05.013

M. Pätzold, S. Tellmann, B. Häusler, D. Hinson, R. Schaa, G.L. Tyler, A sporadic third layer in the ionosphere of Mars. Science 310, 837-839 (2005)

M. Pätzold, B. Häusler, M.K. Bird, S. Tellmann, R. Mattei, S.W. Asmar, V. Dehant, W. Eidel, T. Imamura, R.A. Simpson, G.L. Tyler, The structure of Venus' middle atmosphere and ionosphere. Nature 450, 657-660 (2007). doi:10.1038/nature06239

M. Pätzold, S. Tellmann, B. Häusler, M.K. Bird, G.L. Tyler, A.A. Christou, P. Withers, A sporadic layer in the Venus lower ionosphere of meteoric origin. Geophys. Res. Lett. 36, 5203-5206 (2009)

L.J. Paxton, Pioneer Venus Orbiter Ultraviolet Spectrometer limb observations: Analysis and interpretation of the 166- and 156-nm data. J. Geophys. Res. 90, 5089-5096 (1985)

L.J. Paxton, D.E. Anderson, Far ultraviolet remote sensing of Venus and Mars, in Venus and Mars: Atmospheres, Ionospheres, and Solar Wind Interactions, ed. by J.G. Luhmann, M. Tatrallyay, R.O. Pepin. Geophys. Monogr., vol. 66 (AGU, Washington, 1992). doi:10.1029/GM066p0113

L.J. Paxton, D.E. Anderson, A.I.F. Stewart, Analysis of the Pioneer Venus Ultraviolet Spectrometer Lymanalpha data from near the subsolar region. J. Geophys. Res. 93, 1766-1772 (1988a)

L.J. Paxton, D.E. Anderson, A.I.F. Stewart, Correction to: analysis of the Pioneer Venus Ultraviolet Spectrometer Lyman-alpha data from near the subsolar region. J. Geophys. Res. 93, 11551 (1988b)

J.B. Pechmann, A.P. Ingersoll, Thermal tides in the atmosphere of Venus - comparison of model results with observations. J. Atmos. Sci. 41, 3290-3313 (1984)

J. Peralta, R. Hueson, A. Sánchez-Lavega, G. Piccioni, O. Lanciano, P. Drossart, Characterization of mesoscale gravity waves in the upper and lower clouds of Venus from VEX-VIRTIS images. J. Geophys. Res. 113, E12 (2008). doi:10.1029/2008JE003185

J. Peralta, M.A. López-Valverde, G. Gilli, A. Piccialli, Dayside temperatures in the Venus upper atmosphere from Venus Express/VIRTIS nadir measurements at $4.3 \mu \mathrm{m}$. Astron. Astrophys. 585, A53 (2016). doi:10.1051/0004-6361/201527191

W.D. Pesnell, J. Grebowsky, Meteoric magnesium ions in the Martian atmosphere. J. Geophys. Res. 105, 1695-1708 (2000)

W.D. Pesnell, J. Grebowsky, P.A. Webb, Meteoric Ions in Venus' Atmosphere, AGU Fall Meeting Abstracts, A238 (2004)

K. Peter, M. Pätzold, G. Molina-Cuberos, O. Witasse, F. Gonzalez-Galindo, P. Withers, M.K. Bird, B. Häusler, D.P. Hinson, S. Tellmann, G.L. Tyler, The dayside ionospheres of Mars and Venus: comparing a one-dimensional photochemical model with MaRS (Mars Express) and VeRa (Venus Express) observations. Icarus 233, 66-82 (2014)

J.L. Phillips, A.I.F. Stewart, J.G. Luhmann, The Venus ultraviolet aurora: observations at $130.4 \mathrm{~nm}$. Geophys. Res. Lett. 13, 1047-1050 (1986). doi:10.1029/GL013i010p01047

A. Piccialli et al., Cyclostrophic winds from the Visible and Infrared Thermal Imaging Spectrometer temperature sounding: A preliminary analysis. J. Geophys. Res. 113 (2008)

A. Piccialli, S. Tellmann, D.V. Titov, S.S. Limaye, I.V. Khatuntsev, M. Pätzold, B. Häusler, Dynamical properties of the Venus mesosphere from the radio-occultation experiment VeRa onboard Venus Express. Icarus 217, 669-681 (2012) 
A. Piccialli, F. Montmessin, D. Belyaev, A. Mahieux, A. Fedorova, E. Marcq, J.L. Bertaux, A.C. Vandaele, O. Korablev, Thermal structure of Venus upper atmosphere measured by stellar occultations with SPICAV/Venus Express. Planet. Space Sci. 113(144), 321-335 (2015). doi:10.1016/j.pss.2014.12.009

A. Piccialli et al., $\mathrm{CO}_{2}$ non-LTE limb emissions in Mars' atmosphere as observed by OMEGA/Mars Express. J. Geophys. Res. 121(6), 1066-1086 (2016). 2016. doi:10.1002/2015JE004981

G. Piccioni et al., First detection of hydroxyl in the atmosphere of Venus. Astron. Astrophys. 483, 3 (2008). doi:10.1051/0004-6361:200809761

G. Piccioni et al., Near-IR oxygen nightglow observed by VIRTIS in the Venus upper atmosphere. J. Geophys. Res. 114, E00b38 (2009). doi:10.1029/2008je003133

M. Rengel, P. Hartogh, D. Jarchow, Mesospheric vertical thermal structure and winds on Venus from HHSMT CO spectral-line observations. Planet. Space Sci. 56, 1368-1384 (2008). doi:10.1016/j.pss.2008.07.004

P.G. Richards, J.A. Fennelly, D.G. Torr, EUVAC: a solar EUV Flux Model for aeronomic calculations. J. Geophys. Res. 99(A5), 8981-8992 (1994). doi:10.1029/94JA00518

H. Rishbeth, M. Mendillo, Ionospheric layers of Mars and Earth. Planet. Space Sci. 52, 849-852 (2004)

A. Roldan, M.A. López-Valverde, M. Lopez-Puertas, D.P. Edwards, Non-LTE infrared emissions of $\mathrm{CO}_{2}$ in the atmosphere of Venus. Icarus 147, 11-25 (2000). doi:10.1006/icar.2000.6432

M. Roos-Serote, P. Drossart, Th. Encrenaz, E. Lellouch, R.W. Carlson, K.H. Baines, F.W. Taylor, The thermal structure of the middle Venusian atmosphere from the Galileo/NIMS spectra. Icarus 114, 300-309 (1995)

J. Rosenqvist, E. Lellouch, Th. Encrenaz, G. Pauber, Global circulation in Venus' mesosphere from IRAM CO observations (1991-1994): a tribute to Jan Rosenqvist. Bull. Am. Astron. Soc. 27, 1080 (1995)

W.B. Rossow, A.D. Del Genio, T. Eichler, Cloud-tracked winds from Pioneer Venus OCPP images. J. Atmos. Sci. 47, 2053-2084 (1990)

A. Sánchez-Lavega, S. Lebonnois, T. Imamura, P. Read, D. Luz, The atmospheric dynamics of Venus. Space Sci. Rev. 1-76 (2017). doi:10.1007/s11214-017-0389-x

K. Schafer et al., Infrared Fourier-Spectrometer experiment from Venera-15. Adv. Space Res. 10, 57-66 (1990)

F.P. Schloerb, S.E. Robinson, W.M. Irvine, Observations of CO in the stratosphere of Venus via its $\mathbf{J}=0-1$ rotational transition. Icarus 43, 121-127 (1980)

F. Schmülling, J. Goldstein, T. Kostiuk, T. Hewagama, D. Zipoy, High precision Wind measurements in the upper Venus atmosphere. Bull. Am. Astron. Soc. 32, 1121 (2000)

G. Schubert, General circulation and the dynamical state of the Venus atmosphere, in Venus, ed. by D.M. Hunten, L. Colin, T.M. Donahue, V.I. Moroz (U. Arizona Press, Tucson, 1983), pp. 681-765

G. Schubert, S.W. Bougher, C.C. Covey, A.D. Del Genio, A.S. Grossman, J.L. Hollingsworth, S.S. Limaye, R.E. Young, Venus atmosphere dynamics: a continuing enigma in Exploring Venus as a Terrestrial Planet. AGU Geophysical Monograph, vol. 176, 2007, pp. 101-120

R.W. Schunk, A.F. Nagy, Ionospheres of the terrestrial planets. Rev. Geophys. Space Phys. 18, 813-852 (1980)

K.P. Shah, D.O. Muhleman, G.L. Berge, Measurement of winds in Venus' upper mesosphere based on Doppler shifts of the 2.6-mm ${ }^{12}$ CO line. Icarus 93, 96-121 (1991)

A.V. Shakun, L.V. Zasova, G. Piccioni, P. Drossart, A. Migliorini, Investigation of oxygen $\mathrm{O}_{2}\left(\mathrm{a}^{1} \Delta_{\mathrm{g}}\right)$ emission on the nightside of Venus: nadir data of the VIRTIS-M experiment of the Venus Express mission. Cosm. Res. 48, 232-239 (2010). doi:10.1134/S0010952510030044

B.D. Shizgal, G.G. Arkos, Nonthermal escape of the atmospheres of Venus, Earth, and Mars. Rev. Geophys. 34, 483-505 (1996). doi:10.1029/96RG02213

T.G. Slanger, P.C. Cosby, D.L. Huestis, T.A. Bida, Discovery of the atomic oxygen green line in the Venus night airglow. Science 291, 463-465 (2001)

T.G. Slanger, D.L. Huestis, P.C. Cosby, N.J. Chanover, T.A. Bida, The Venus nightglow: ground-based observations and chemical mechanisms. Icarus 182, 1 (2006). doi:10.1016/j.icarus.2005.12.007

T.G. Slanger, N.J. Chanover, B.D. Sharpee, T.A. Bida, $\mathrm{O} / \mathrm{O}_{2}$ emissions in the Venus nightglow. Icarus 217, 845-848 (2012)

G. Sonnabend, M. Sornig, R. Schieder, T. Kostiuk, J. Delgado, Temperatures in Venus upper atmosphere from mid-infrared heterodyne spectroscopy of $\mathrm{CO}_{2}$ around $10 \mu \mathrm{m}$ wavelength. Planet. Space Sci. 56, 1407-1413 (2008). doi:10.1016/j.pss.2008.05.008

G. Sonnabend, P. Kroetz, M. Sornig, D. Stupar, Direct observations of Venus upper mesospheric temperatures from ground based spectroscopy of $\mathrm{CO}_{2}$. Geophys. Res. Lett. 37 (2010). doi:10.1029/2010GL043335

L. Soret, J.-C. Gérard, Is the $\mathrm{O}_{2}\left(\mathrm{a}^{1} \Delta_{\mathrm{g}}\right)$ Venus nightglow emission controlled by solar activity? Icarus $\mathbf{2 6 2}$, 170-172 (2015). doi:10.1016/j.icarus.2015.08.030

L. Soret, J.-C. Gérard, G. Piccioni, P. Drossart, Venus OH nightglow distribution based on VIRTIS limb observations from Venus Express. Geophys. Res. Lett. 37, L06805 (2010). doi:10.1029/2010GL042377 
L. Soret, J.-C. Gérard, F. Montmessin, G. Piccioni, P. Drossart, J.L. Bertaux, Atomic oxygen on the Venus nightside: global distribution deduced from airglow mapping. Icarus 217, 849-855 (2012a). doi:10.1016/j.icarus.2011.03.034

L. Soret, J.-C. Gérard, G. Piccioni, P. Drossart, The OH Venus nightglow spectrum: intensity and vibrational composition from VIRTIS-Venus Express observations. Planet. Space Sci. 73, 1 (2012b). doi:10.1016/j.pss.2012.07.027

L. Soret, J.-C. Gérard, G. Piccioni, P. Drossart, Time variations of $\mathrm{O}_{2}\left(\mathrm{a}^{1} \Delta\right)$ nightglow spots on the Venus nightside and dynamics of the upper mesosphere. Icarus 237, 306-314 (2014). doi:10.1016/ j.icarus.2014.03.034

M. Sornig, T. Livengood, G. Sonnabend, P. Kroetz, D. Stupar, T. Kostiuk, R. Schieder, Venus upper atmosphere winds from ground-based heterodyne spectroscopy of $\mathrm{CO}_{2}$ at $10 \mu \mathrm{m}$ wavelength. Planet. Space Sci. 56, 1399-1406 (2008). doi:10.1016/j.pss.2008.05.006

K. Spenner, W.C. Knudsen, W. Lotze, Suprathermal electron fluxes in the Venus nightside ionosphere at moderate and high solar activity. J. Geophys. Res. 101, 4557-4563 (1996). doi:10.1029/95JE03495

P.G. Steffes, J.M. Jenkins, R.S. Austin, S.W. Asmar, D.T. Lyons, E.H. Seale, G.L. Tyler, Radio occultation studies of the Venus atmosphere with the Magellan spacecraft. 1: Experimental description and performance. Icarus 110, 71-78 (1994)

G.I. Stepanova, G.M. Shved, Radiative transfer in the 4.3-micron $\mathrm{CO}_{2}$ and 4.7-micron $\mathrm{CO}$ bands in the nonLTE Venus and Mars atmospheres, Transformation of absorbed solar energy. Sov. Astron. 29, 528-531 (1985)

S.A. Stern, D.C. Slater, G.R. Gladstone, E. Wilkenson, W.C. Cash, J.C. Green, D.M. Hunten, T.C. Owen, L. Paxton, The 825-1110 ̊̊ EUV spectrum of Venus. Icarus 122, 200-204 (1996). doi:10.1006/ icar.1996.0119

M.H. Stevens et al., New observations of molecular nitrogen in the Martian upper atmosphere by IUVS on MAVEN. Geophys. Res. Lett. 42, 9050-9056 (2015). doi:10.1002/2015GL065319

A.I.F. Stewart, J.-C. Gérard, D.W. Rusch, S.W. Bougher, Morphology of the Venus ultraviolet night airglow. J. Geophys. Res. 85, 7861-7870 (1980). doi:10.1029/JA085iA13p07861

A. Stiepen, L. Soret, J.-C. Gérard, C. Cox, J.L. Bertaux, The vertical distribution of the Venus NO nightglow: limb profiles inversion and one-dimensional modeling. Icarus 220, 981-989 (2012). doi:10.1016/ j.icarus.2012.06.029

A. Stiepen, J.-C. Gérard, M. Dumont, C. Cox, J.L. Bertaux, Venus nitric oxide nightglow mapping from SPICAV nadir observations. Icarus 226, 428-436 (2013). doi:10.1016/j.icarus.2013.05.031

H.A. Taylor, H.C. Brinton, S.J. Bauer, R.E. Hartle, P.A. Cloutier, R.E. Daniell, T.M. Donahue, Ionosphere of Venus - first observations of day-night variations of the ion composition. Science 205, 96-99 (1979a)

F.W. Taylor, D.J. Diner, L.S. Elson, D.J. McCleese, J.V. Martonchik, J. Delderfield, S.P. Bradley, J.T. Schofield, J.C. Gille, M.T. Coffey, Temperature, cloud structure, and dynamics of Venus middle atmosphere by infrared remote sensing from Pioneer Orbiter. Science 205, 65-67 (1979b)

F.W. Taylor, R. Beer, M.T. Chahine, D.J. Diner, L.S. Elson, R.D. Haskins, D.J. McCleese, J.V. Martonchik, P.E. Reichley, S.P. Bradley, J. Delderfield, J.T. Schofield, C.B. Farmer, L. Froidevaux, J. Leung, M.T. Coffey, J.C. Gille, Structure and meteorology of the middle atmosphere of Venus: infrared remote sensing from the Pioneer Orbiter. J. Geophys. Res. 85, 7963-8006 (1980)

S. Tellmann, M. Pätzold, B. Häusler, M.K. Bird, G.L. Tyler, Structure of the Venus neutral atmosphere as observed by the Radio Science experiment VeRa on Venus Express. J. Geophys. Res. 114, E00B36 (2009). doi:10.1029/2008JE003204

S. Tellmann, B. Häusler, D.P. Hinson, G.L. Tyler, T.P. Andert, M.K. Bird, T. Imamura, M. Pätzold, S. Remus, Small-scale temperature fluctuations seen by the VeRa Radio Science Experiment on Venus Express. Icarus 221, 471-480 (2012)

S. Tingle S, The Dynamics of the Venusian Mesosphere and Thermosphere. Ph.D. Thesis, Imperial College London (2011)

D.V. Titov et al., Morphology of the cloud tops as observed by the Venus Express Monitoring Camera. Icarus 217, 682-701 (2012)

W.K. Tobiska, T. Woods, F. Eparvier, R. Viereck, L. Floyd, D. Bouwer, G. Rottman, O.R. White, The SOLAR2000 empirical solar irradiance model and forecast tool. J. Atmos. Sol.-Terr. Phys. 62(14), 12331250 (2000)

M.B. Vasilev, A.S. Vyshlov, M.A. Kolosov, A.P. Mesterton, N.A. Savich, V.A. Samovol, L.N. Samoznaev, A.I. Sidorenko, Two-frequency radio occultation measurements with Venera-9 and Venera-10 orbiters. Acta Astronaut. 7, 335-340 (1980)

U. von Zahn, K.H. Fricke, H. Hoffmann, K. Pelka, Venus-Eddy coefficients in the thermosphere and the inferred helium content of the lower atmosphere. Geophys. Res. Lett. 6, 337-340 (1979)

U. von Zahn, S. Kumar, H. Niemann, R. Prinn, Composition of the Venus atmosphere. in Venus, ed. by D.M. Hunten, L. Colin, T.M. Donahue, V.I. Moroz (The University of Arizona Press, Tucson, 1983) 
T. Widemann, E. Lellouch, A. Campargue, New wind measurements in Venus's lower mesosphere from visible spectroscopy. Planet. Space Sci. 55, 1741-1756 (2007). doi:10.1016/j.pss.2007.01.005

O. Witasse, A.F. Nagy, Outstanding aeronomy problems at Venus. Planet. Space Sci. 54, 13-14 (2006). doi:10.1016/j.pss.2006.04.028

P. Withers, A.A. Christou, J. Vaubaillon, Meteoric ion layers in the ionospheres of Venus and Mars: early observations and consideration of the role of meteor showers. Adv. Space Res. 52, 1207-1216 (2013)

R. Woo, A.J. Kliore, Magnetization of the ionospheres of Venus and Mars-results from radio occultation measurements. J. Geophys. Res. 96, 11073 (1991)

T.N. Woods, G. Rottman, Solar ultraviolet variability over time periods of aeronomic interest, in Atmospheres in the Solar System. Geophysical Monograph, vol. 130 (American Geophysical Union, Washington, 2002), pp. 221-233

A.M. Zalucha, A.S. Brecht, S. Rafkin, S.W. Bougher, M.J. Alexander, Incorporation of a gravity wave momentum deposition parameterization into the VTGCM. J. Geophys. Res. 118 (2013). doi:10.1029/ 2012JE004168

S. Zhang, S.W. Bougher, M.J. Alexander, The impact of gravity waves on the Venus thermosphere and $\mathrm{O}_{2}$ IR nightglow. J. Geophys. Res. 101, 23195-23205 (1996)

T.L. Zhang et al., Initial Venus Express magnetic field observations of the magnetic barrier at solar minimum. Planet. Space Sci. 56, 790-795 (2008) 